Portland State University

PDXScholar

$6-24-2020$

\title{
Examining the Narratives of Military Sexual Trauma Survivors
}

Maria Carolina González-Prats

Portland State University

Follow this and additional works at: https://pdxscholar.library.pdx.edu/open_access_etds

Part of the Military and Veterans Studies Commons, and the Social Work Commons Let us know how access to this document benefits you.

\section{Recommended Citation}

González-Prats, Maria Carolina, "Examining the Narratives of Military Sexual Trauma Survivors" (2020). Dissertations and Theses. Paper 5511.

https://doi.org/10.15760/etd.7385

This Dissertation is brought to you for free and open access. It has been accepted for inclusion in Dissertations and Theses by an authorized administrator of PDXScholar. Please contact us if we can make this document more accessible: pdxscholar@pdx.edu. 
Examining the Narratives of Military Sexual Trauma Survivors

by

Maria Carolina González-Prats

A dissertation submitted in partial fulfillment of the requirements for the degree of

Doctor of Philosophy

in

Social Work and Social Research

Dissertation Committee:

Christina Nicolaidis, Chair

Gita Mehrotra

Katharine Cahn

Greg Townley

Portland State University

2020 
C 2020 Maria Carolina González-Prats 


\begin{abstract}
Background: The return of military sexual trauma (MST) to the national spotlight has been fueled by a combination of continued reports of sexual assaults across the various military branches, increased visibility of sexual abuse scandals in the media, and mounting calls for accountability from veteran advocacy groups and legislators. Although there have been numerous reforms implemented by the military, there still exists a significant gap still exists between the military's official efforts and the reality of the survivors' experiences. Consequently, more research is needed to understand how survivors perceive the military's efforts, how these efforts affect their experiences, and how future MST prevention and response programs can be improved.

Methods: This qualitative study was based on open-ended and semi-structured interviews with a national sample of 21 Army, Navy, and Air Force servicemembers who experienced sexual harassment and/or sexual assault in the military between 2003 and 2019. We conducted a thematic analysis with a secondary coder to identify semantic and latent themes within the lived experiences of MST survivors.

Findings: Four key findings that emerged from the interviews: (a) the military's culture of sexism and misogyny contributes to MST, (b) the leadership and the chain of command matters, (c) response efforts were often (re)traumatizing; and (d) prevention efforts, particularly training, are important and often inadequate. Additionally, the participants provided eight recommendations for military leaders to improve the culture, as well as their MST prevention and response efforts.
\end{abstract}


Conclusion: The study findings suggest that the MST prevention/response strategies needs to (a) be consistent with military values and culture; b) be balanced with consistent accountability and military justice policies, and (c) ensure that prevention training facilitators have a robust skillset to deal with difficult/sensitive subject matter.

Additionally, participants pointed out examples of what a survivor-centered prevention and response strategy could look like.

Implications: This qualitative study provides a baseline for future MST research in the area of sexual assault and prevention programming in both the military and veteran communities. This study also offers civilian practitioners in the fields of social work and social work education additional insight into sexual trauma within the context of the military culture. 


\section{Acknowledgements}

There are dozens of people and several institutions that supported me and helped make this study and the Ph.D. journey possible. Foremost, I express my appreciation to the 21 survivors who bravely participated in this study and generously shared their experiences with me. Also, five individuals were instrumental during the earlier stages of consultation, participant outreach, data collection, and analysis: Aimee “AJ” LaFevre, Erin Danielson (United States Marine Corps), Alison Rae Taimanglo-Cuasay (United States Army), and Emma Arnesty-Good. I would also like to recognize PSU's Regional Research Institute (RRI) for the administrative and information technology support I received for recruiting/onboarding my secondary coder and for participant outreach and recruiting.

The entire dissertation process benefitted from the tireless mentorship, leadership and encouragement from my dissertation chairwoman, Dr. Christina Nicolaidis, as well as the insight and coaching from my dissertation committee members, Dr. Katharine Cahn, Dr. Gita Mehrotra and Dr. Greg Townley. To the faculty and students in the PSU School of Social Work who continue to inspire, encourage and serve, especially the support my 2013 doctoral cohort "Fab 5," and fellow Ph.D. student writing partner and great friend, Martha McCormack provided. Additionally, I greatly appreciate the endless optimism and leadership of our Ph.D. program director Dr. Junghee Lee, and the seamless logistical support from our Social Work MSW/Ph.D. Administration Specialist, Thet Mar Win. 
To my parents Martha Perez-Arostegui and Enrique González-Prats, who inspired my curiosity and passion for adventure and lifelong learning. To my entire family who has weathered this $\mathrm{Ph} . \mathrm{D}$. journey with me with enthusiasm, humor, and patience, I say thank you. I would especially like to recognize my two sisters: Vicky Ascoli, who sacrificed extra work and personal time to accommodate my countless hours of uninterrupted writing and editing, and my sister Maria Elena Campisteguy, who has been one of my most vocal cheerleaders of my academic and professional journey after I got out of the Army in 2004.

Lastly, this dissertation was supported by the Oregon Clinical and Translational Research Institute (OCTRI), grant number (TL1 TR 002371), from the National Center for Advancing Translational Sciences at the National Institutes of Health. The content is solely the responsibility of the authors and does not necessarily represent the official views of the NIH. I appreciate the mentorship, coaching, administrative, and participant recruitment support I received from OCTRI during the course of the research for my study. 


\section{Table of Contents}

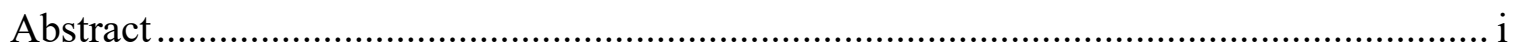

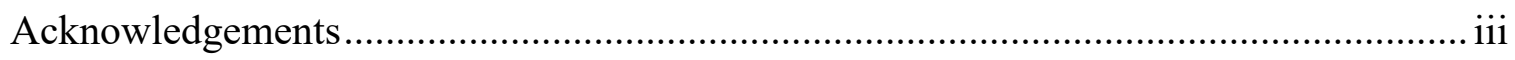

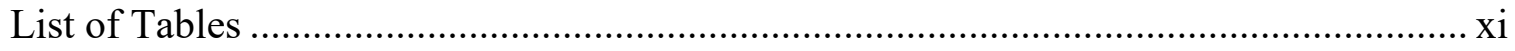

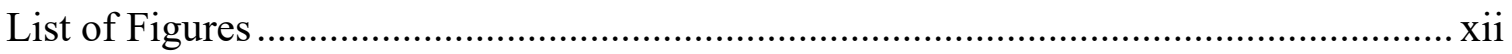

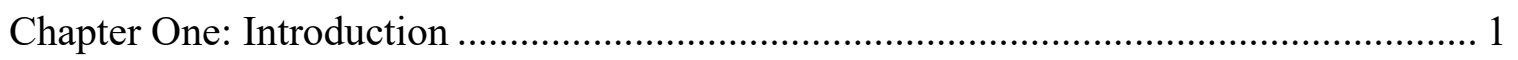

Part I: General Overview of Military Sexual Trauma ............................................... 3

What Is Military Sexual Trauma? .................................................................... 3

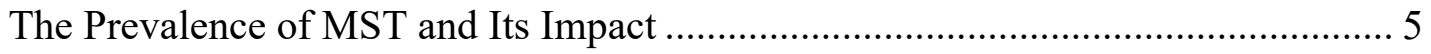

Effects of Military Sexual Trauma ….......................................................... 9

Why is Military Sexual Trauma a Social Problem?.......................................... 10

What's Missing from the Military's Prevention and Response Efforts ................... 11

Part II: Overview of Study/Purpose .................................................................. 14

Contributions to Field of Social Work and Social Research ............................... 15

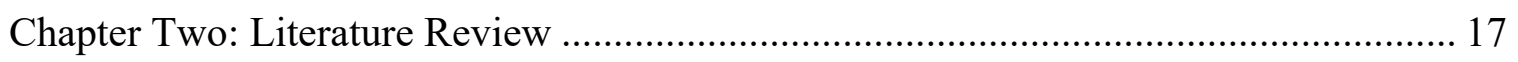

Conceptual Framework Guiding This Study ..................................................... 18

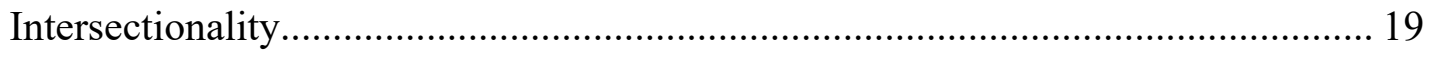

Gender Identity Constructs, Sexual Harassment \& Sexual Assault .......................... 21

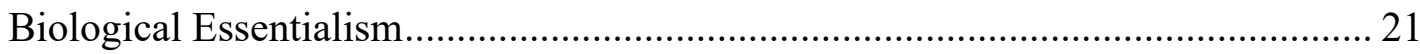

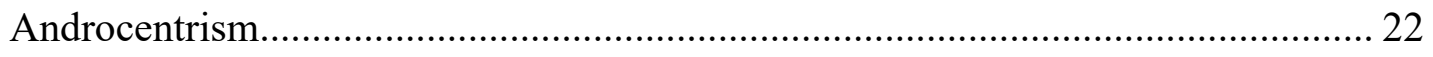

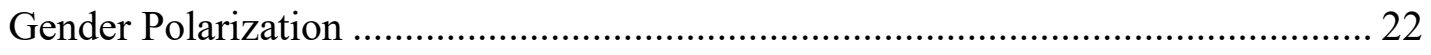

The Intersection of Gender, Sexual Harassment, and Sexual Assault........................ 25 
Ambivalent Sexism and Rape Myth Acceptance: A Review of the Literature ........... 26

Messaging, Sexual Harassment, and Sexual Assault................................................. 33

The Framing of Sexual Violence in our Culture................................................ 35

The Foci of Sexual Violence Prevention Programming ........................................ 39

Recommended Communication Strategies for Sexual Violence Prevention............... 43

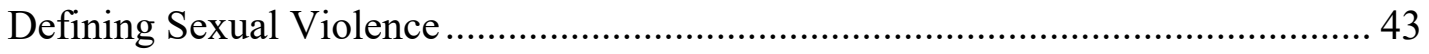

Public Health Approach to Sexual Violence ......................................................... 44

Applying the Lessons Learned from the Anti-Drunk Driving Movement to Sexual

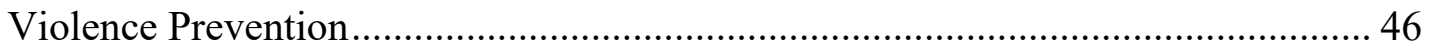

Gender Identity and Messaging in the Military .................................................. 48

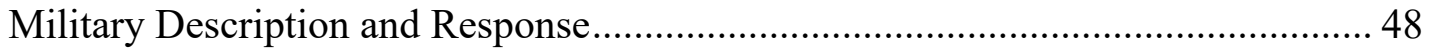

Department of Defense Military Sexual Trauma Reports .................................. 49

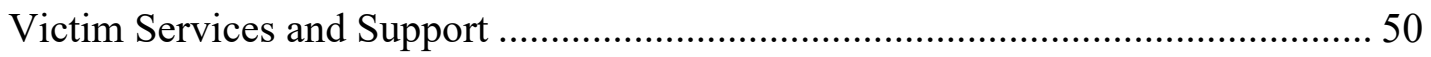

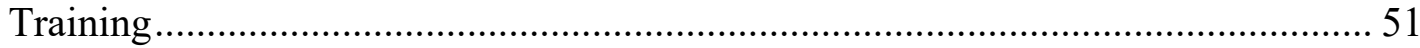

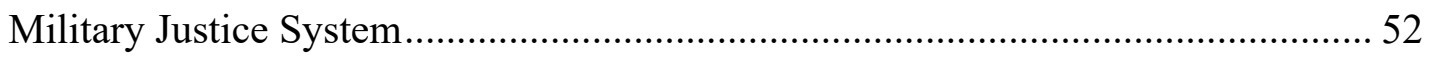

Gap Between the Department of Defense's Data and the Survivor Experience........... 53

Lack of Accountability and Retaliation ...................................................... 54

Gender Identity and Military Culture …........................................................ 55

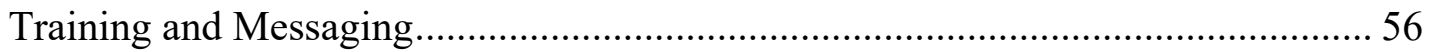

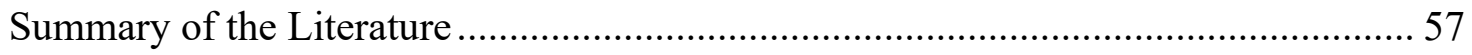

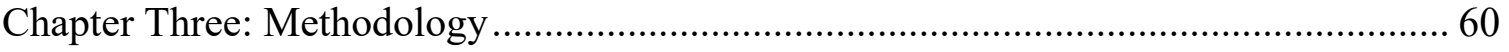

My Positionality as a Researcher/ Research Approach .......................................... 60 


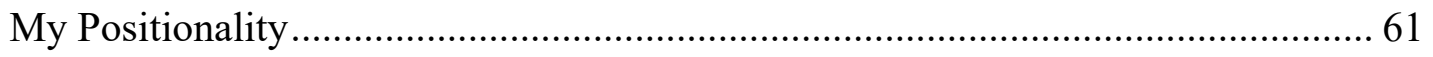

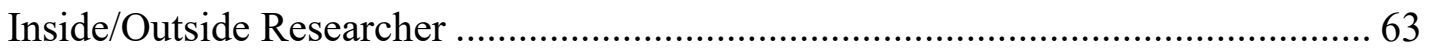

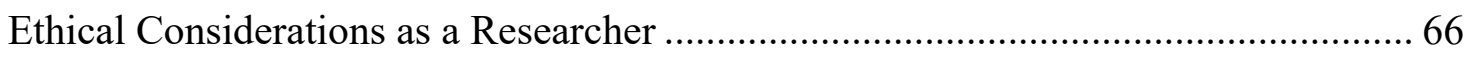

Weighing the Risk and Benefits of Trauma-Related Research ............................. 67

Implication for Research and Further Studies .............................................. 69

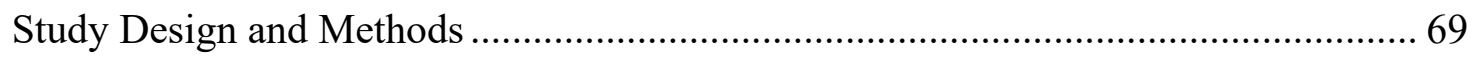

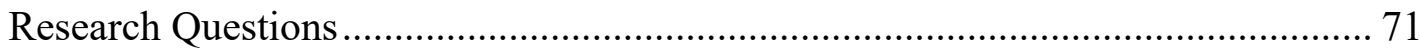

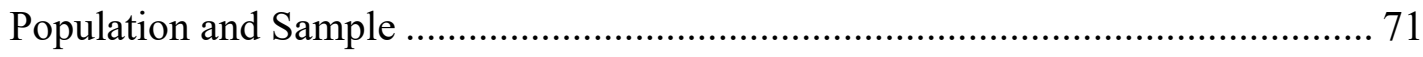

The Process of Data Collection and Analysis............................................................ 72

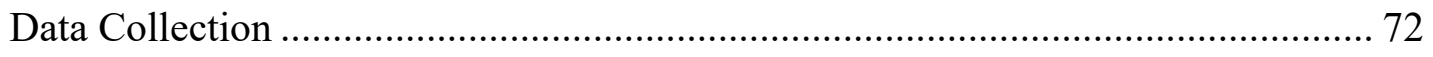

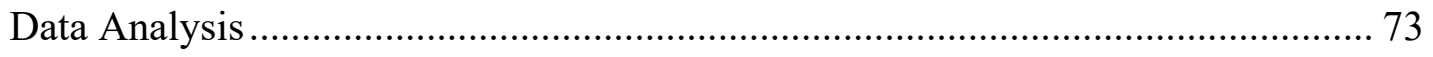

Proposed Next Steps in Increasing the Trustworthiness and the Validity of This

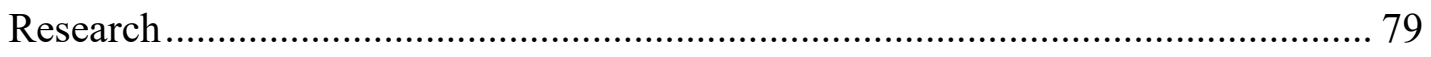

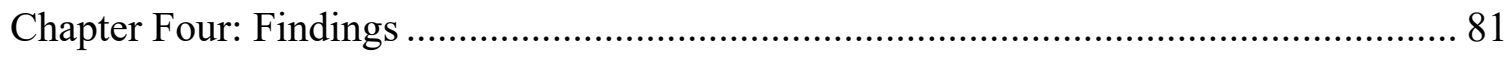

Theme One: The Military's Culture of Sexism and Misogyny Contribute to MST ..... 86

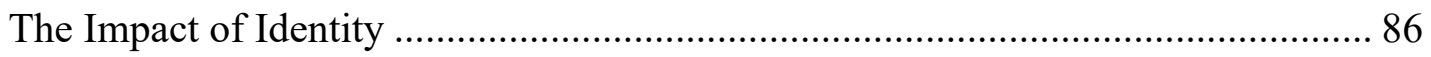

Survivors’ Experiences with MST are Minimized/Ignored Within Romantic

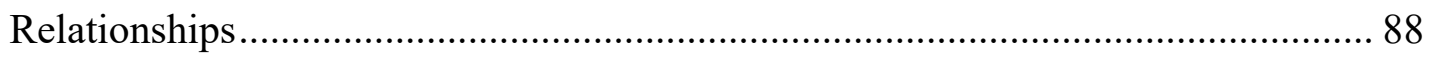

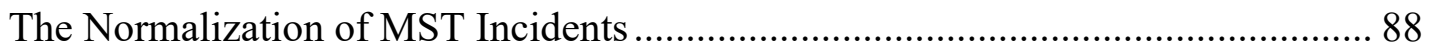

Victims Were Often Bullied and Slut-Shamed .................................................. 91

Theme Two: The Leadership and Chain of Command Matters ............................... 93

Leaders are Responsible for the Command Climate of their Military Unit.............. 94 
Inconsistent Treatment for Women Versus Men by the Leadership 96

Survivors Experienced Professional Retaliation by Leadership for Rejecting Advances and/or Reporting Incidents 98

The Career of the Perpetrator is often Prioritized Over That of the Victims 101 What a Positive and Supportive Military Environment Looks Like to a Survivor. 102

Theme Three: Response Efforts Were Often (Re)-Traumatizing. 106 MST Has a Lifelong Impact on the Survivors 106 Survivors Were Often Treated Poorly by Military and VA Health Care Providers 109 Discriminatory Policies like “Don’t Ask Don’t Tell” Can Deter Reporting Incidents of MST 113

Theme Four: Prevention Efforts, In Particular Training, are Important and Often

Inadequate 114

The Perceived Quality of the Training Content.................................................. 114

The Delivery of the Information Matters (ex. Timing, Methods, etc.)................... 116

The Skills of the Facilitators are Key ................................................................... 118

Recommendations from Survivors ............................................................. 120

Recommendation 1: Don't use Gender Segregation to Prevent MST .................... 120

Recommendation 2: Lead by Example (hold leaders to high standards of professionalism)

Recommendation 3: Increase the Consistency of Accountability for Perpetrators of MST 123 
Recommendation 4: Transfer investigative authority outside the Chain of Command

Recommendation 5 Treat MST as Seriously as any Other Injury .....

Recommendation 6: Improve Medical/Psychological Support Protocols for Military

Servicemembers and Veterans

128

Recommendation 7: Expand MST Prevention Training Content for Servicemembers

and Leaders

129

Recommendation 8: Improve the Quality of the SAPR Staff/Trainers/Facilitators

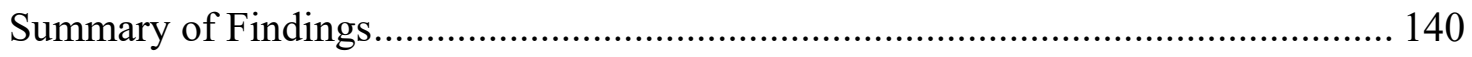

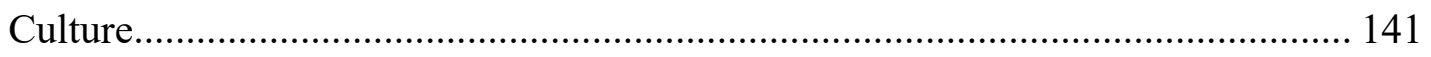

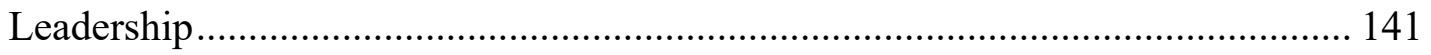

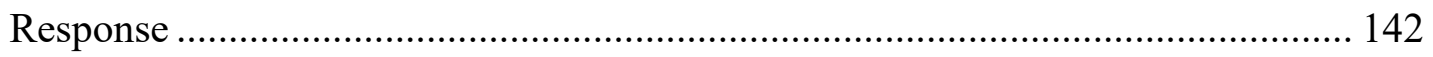

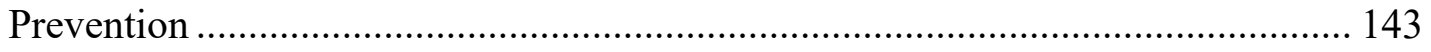

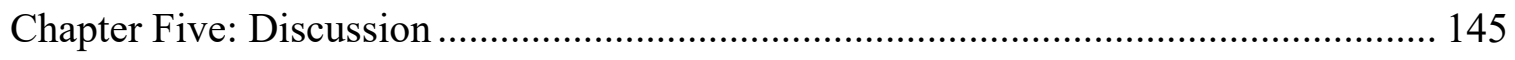

Key Takeaways from the Study Findings ........................................................ 145

How This Study Moves the Literature Forward ............................................... 148

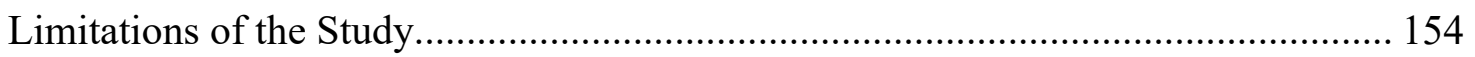

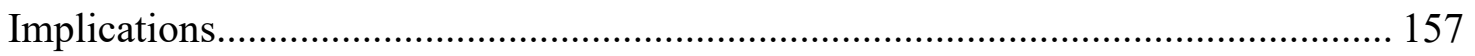

Military and Veteran Communities ............................................................. 157

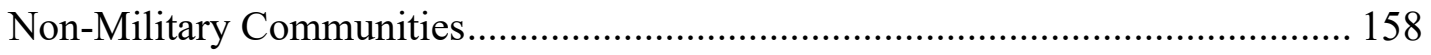

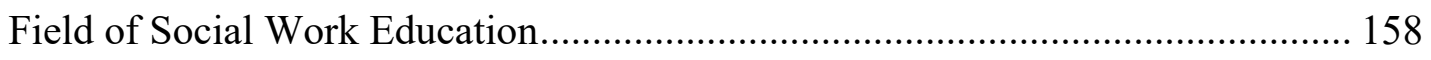


Future Research ...................................................................................... 158

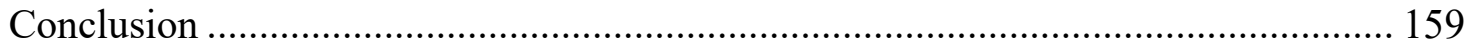

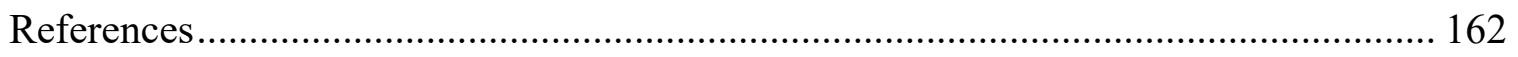

Appendix A: Participant Screening Tool …...................................................... 188

Appendix B: Informed Consent, Phone Interview.................................................. 198

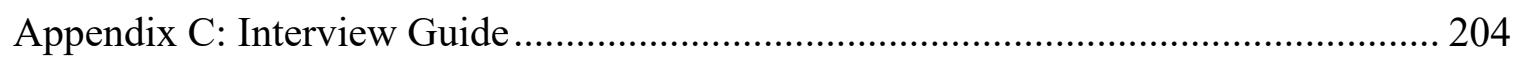




\section{List of Tables}

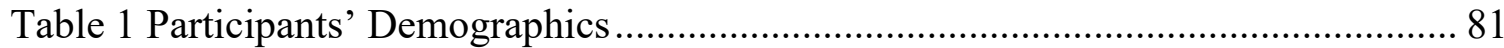

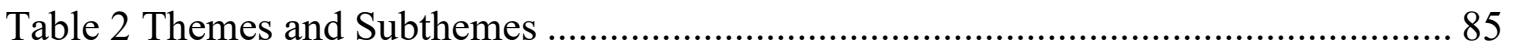

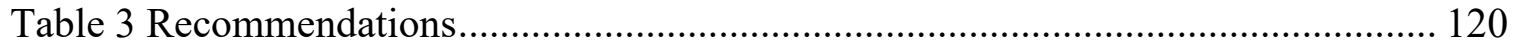




\section{List of Figures}

Figure 1. 2017 annual report statistical highlights. .................................................. 7

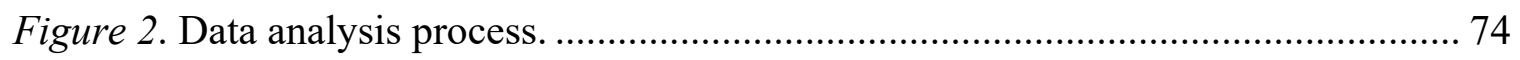

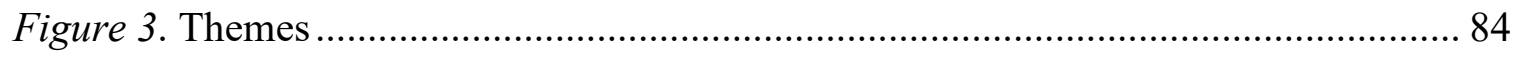




\section{Chapter One: Introduction}

Within the year prior to this study, the United States experienced a wave of cultural backlash and public outcry against acts of sexual harassment and sexual assault as well as the perpetrators who commit them. An increased number of well-known and prominent figures in the public and private sectors are being held accountable for abusing their power and position to sexually harass, abuse, and assault. Additionally, survivors and victim ${ }^{1}$ advocacy groups have called attention to the practice of threats and retaliatory actions to silence and intimidate victims from coming forward and reporting the incidents of abuse.

From the Hollywood entertainment industry to start-up and tech companies in Silicon Valley to mass media and American politics (Garcia, 2017; Sumagaysay, 2017; Zacharek, Docterman, \& Sweetland Edwards, 2017), there has been a public outcry and demand for reforms and increased accountability for perpetrators and for justice for survivors of sexual harassment and sexual assault. Known as the \#metoo ${ }^{2}$ movement (Garcia, 2017), numerous survivors have felt emboldened to share their stories of victimization and abuse, consequently empowering other survivors to do the same (Garcia, 2017; Sumagaysay, 2017; Zacharek et al., 2017).

An additional outcome of the \#metoo movement has been a renewed public debate about the responsibility that organizations and institutions in the public and private

\footnotetext{
1 In this dissertation, the term 'survivor' will be used interchangeably with the term 'victim', acknowledging that some individuals choose to identify as one or the other (Hannagan, 2016).

${ }^{2}$ Although the \#metoo movement gained notoriety with a tweet from actress Alyssa Milano, the term was originally coined by activist Tarana Burke in the late 1990s.
} 
sectors have in creating and sustaining a culture that condones or enables incidents of discrimination, sexual harassment and abuse in the workplace as well as the organization's level of liability in failing to protect employees in the workplace (Sumagasay, 2017). This is particularly important when the public institution is significantly dependent on the participation of an all-volunteer labor force and entrusted with national defense. Although the military is mostly on the periphery of the \#metoo movement (Rico, 2017; Seck, 2018), it serves as an example of a segment of the U.S. workforce that continues to face incidents of sexual abuse within its ranks despite its more than 30 years' worth of claims of an environment of strict accountability and zero tolerance (Dick, 2012; Hunter, 2007; Januta, n.d.; Solaro, 2006,).

In the 15 to 20 years since Tailhook, Aberdeen Proving Grounds, and the Air Force Academy sexual abuse scandals that broke in the 1990s and early 2000, it is apparent that the military's prevention and response strategies have not been effective in reversing the trajectory of sexual harassment and sexual assault (González-Prats, 2017). To date, the military's efforts to address military sexual trauma (MST) have been focused on prevention training and improving support services for sexual assault victims. However, what is missing from the military's current prevention and response efforts is a comprehensive and consistent communication strategy regarding incidents of sexual harassment and sexual assault that is congruent with its culture of strict accountability and discipline.

Combined with the continued reports of sexual assaults across the military branches of service, the increased visibility of sexual abuse scandals in the media and 
mounting calls for accountability from veteran advocacy groups and legislators, the issue of MST has gained national attention (Baldor, 2018; Brangham \& Woodruff, 2018; Cohen \& Browne, 2018).

This chapter is bifurcated into two sections: a brief overview of MST and an introduction to the dissertation study.

\section{Part I: General Overview of Military Sexual Trauma}

In this section, I present a brief background of what is known about MST, such as the definition, whom it impacts, its prevalence, and why it should be considered a collective social problem.

\section{What Is Military Sexual Trauma?}

The Department of Veteran Affairs defines MST as sexual harassment and sexual assault incurred during military service (United States Department of Veterans Affairs, 2017). The military defines sexual harassment as

a form of sex discrimination that involves unwelcome sexual advances, requests for sexual favors, and other verbal or physical conduct of a sexual nature when: a) submission to such conduct is made either explicitly or implicitly a term or condition of a person's job, pay, or career, or b) submission to or rejection of such conduct by a person is used as a basis for career or employment decisions affecting that person, or c) such conduct has the purpose or effect of unreasonably interfering with an individual's work performance or creates an intimidating, 
hostile, or offensive working environment ${ }^{3}$ (Department of Defense [DoD], 2014b, p. 1).

The military can charge a service member with sexual assault if they cause another person of any age to engage in a sexual act by: 1) using force against that other person 2) causing grievous bodily harm to any person 3) threatening or placing that other person in fear that any person will be subjected to death, grievous bodily harm, or kidnapping 4) rendering another person unconscious or 5) administering to another person by force or threat of force, or without the knowledge or permission of that person, a drug, intoxicant, or similar substance and thereby substantially impairs the ability of that other person to appraise or control conduct. (Joint Service Committee on Military Justice, 2008, p. 351).

Military sexual trauma occurs across the branches and various service components of the Army, Navy, Air Force, Marine Corps, and the Coast Guard ${ }^{4}$, including in active duty, reserves, and the National Guard as well as the military service academies (Defense Manpower Data Center [DMDC], 2012; DoD, 2014c; Veterans Health Care Administration, 2013).

Historically, both sexual assault and sexual harassment have been discussed and tracked separately as two independent and unrelated offenses. However, the most recent and significant shift has been the acknowledgement by the DoD that sexual harassment

\footnotetext{
${ }^{3}$ Military workplace includes conduct on or off duty, 24 hours a day 4 MST also refers to trauma incurred in the Coast Guard, although this branch doesn't fall under the Department of Defense, but the Department of Homeland Security
} 
and sexual assault run along a "continuum of harm" (DoD, 2014a, p. 21) that ranges from gender-focused jokes, sexual comments, and vulgar pictures to physical force, sexual fondling, forcible sodomy, and rape (DoD, 2014d, 2016a).

For the purposes of protecting the integrity of the term MST and of promoting continuity throughout this chapter, I will only refer to MST when referring to sexual harassment and sexual assault combined and, as appropriate, refer to military sexual harassment (MSH) or military sexual assault (MSA) individually.

\section{The Prevalence of MST and Its Impact}

Prior to considering the data regarding MST, it is important to note the prevalence (Hulley, 2014) of data provided by the DoD is compartmentalized, as it was collected by various agencies with differing terminology (i.e., unwanted sexual contact, sexual assault, sexual harassment) and frequency of reporting (i.e., every fiscal year, every academic program year, biennially, every four calendar years, and so forth).

The information is disaggregated across the active duty components, the reserve service components, and the military service academies. Additionally, the National Guard Bureau and Coast Guard Bureau are selectively included in some of the aggregate data, but that is not always explicitly stated or obvious to the average citizen accessing the information. In short, it is difficult to form a big picture view of MST, its continued prevalence, and severity of the problem across the branches of service and the military as a whole. It is equally as difficult to strategically develop effective prevention and response efforts (Rosenthal \& Miller, 2013). 
Military sexual assault (MSA). It is estimated that 1 in 5 women and 1 in 100 men in the military are sexually assaulted (United States Department of Veteran Affairs, 2016). These rates of MSA closely resemble the rates in the civilian community. According to the Rape and Incest National Network (RAINN, 2009), 1 in 6 women, and 1 in 33 men have experienced an attempted or completed rape in their lifetime.

The victims who are at increased risk for sexual violence are members of the American Indian/Native American5 communities (Breiding, 2014; Planty, Langon, Krebs, Berzofsky, \& Smiley-McDonald, 2013; Department of Justice, 2004; RAINN, 2009) and members of the lesbian, gay, bisexual, transgender, and queer (LGBTQ) community ${ }^{6}$ (Cantor et al., 2015; Human Rights Watch, 2015; RAINN, 2009; Walters, Chen, \& Breiding, 2013). As with the military, these rates are believed to be conservative as sexual assault/rape are violent crimes that are significantly underreported (RAINN, 2009).

Female service members receive the majority of the public's attention, news coverage, and targeted messaging about MSA. This is despite the fact that men make up about $80 \%$ to $85 \%$ of the active duty and reserve military forces and represent about $54 \%$ of the total number of victims who screen positively for MSA by the Veterans Administration (DoD, 2015a, 2015b; Hoyt, Klosterman Rielage, \& Williams, 2011; Office of the Deputy Assistant Secretary of Defense, 2014; Steward, 2013).

5 American Indians/Native American women have the highest rate of rape/sexual assault victimization, compared to other races.

6 Bisexual women and transgender college students are disproportionately impacted by sexual violence. 
As shown below in Figure 1 from the 2017 Annual Report Statistical Highlights, there was a significant increase of reports between fiscal year 2012 and fiscal year 2017 (DoD, 2018). The military claims that the increase in sexual assault reports is reflective of the victims' increased confidence in the military justice system, rather than an increase in sexual assaults (Baldor, 2018; DoD, 2015a, 2015b; RAND National Defense Research Institute, 2014). However, there is no conclusive evidence to support the military's assertion that the increased rates are due to an increase in reporting versus an increase in crime (Protect Our Defenders, n.d.; Rosenthal \& Miller, 2013) ${ }^{7}$

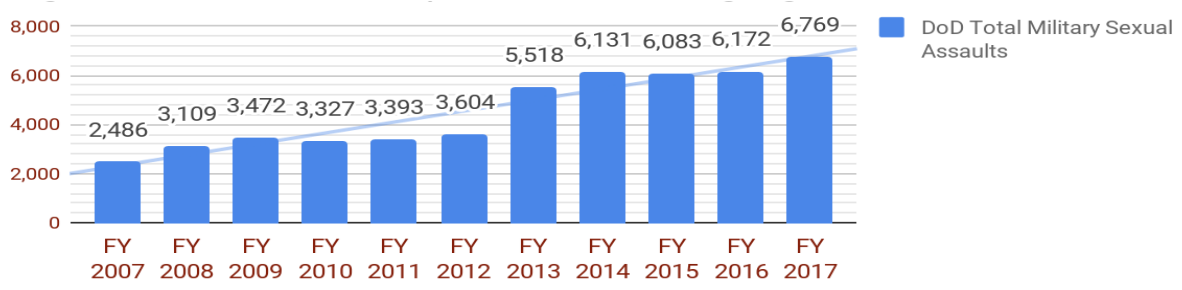

Figure 1. 2017 annual report statistical highlights.

Military sexual harassment (MSH). While the systems in place for tracking sexual assault cases are fairly uniform across the Armed Forces, the existing guidelines and policies for the sexual harassment complaint process directed by the DoD Military Equal Opportunity Program provide a limited amount of flexibility across the military branches to accommodate the differences in organizational culture and mission (DMDC, 2012, 2014).

7 It is important to note that the above data do not include Coast Guard personnel, any cadets or midshipmen in the military service academies, nor any service members forward deployed in areas to include, but not limited to Iraq, Afghanistan, and Kuwait, referred by the military as Combat Areas of Interest (CAI); these sexual assault cases are tracked in separate reports and/or by a different government agency, which are detailed below in the different measurements section. 
In fiscal year $2013,{ }^{8}$ the Military Services and National Guard Bureau reported 1,366 formal $^{9}$ and informal ${ }^{10}$ sexual harassment complaints. Of these claims, $59 \%$ were substantiated $^{11}, 29.5 \%$ were unsubstantiated ${ }^{12}$, and $9.2 \%$ were pending resolution/investigation at the end of the fiscal year. Especially noteworthy are the alleged repeat offenders, which made up $11 \%$ of formal sexual harassment complaints, and of which $72 \%$ were substantiated. Complaints that involved the same offenders comprised $14 \%$ of informal sexual harassment complaints, of which $35 \%$ were substantiated (DoD, 2016). Additionally, the military service academies reported an increase from 20 sexual harassment complaints during the 2013-2014 term to 34 complaints in the 2016-2017 term (DoD, 2018).

The method of calculating or quantifying the incidence of MST has been a source of extensive debate, leading to consternation in the military, veteran, and civilian communities alike. In turn, the prevention and response efforts of the military are based on these prevalence rates, raising a classic conundrum. On one hand, DoD leaders would like to show the public how committed they are to eradicate sexual assault by tracking the sexual assault rates every fiscal year. On the other hand, they demonstrate distrust of these same statistics, claiming that they are an overstatement of the problem and that

8 As of May 2018, there is not an updated DoD Report of comprehensive sexual harassment rates available beyond FY 2013

9 An allegation that can be made verbally or in writing and is submitted through the Military Equal Opportunity Office (MEO) or their chain of command

10 An allegation that can be made in writing or verbally and it is not submitted through the MEO

11 Sexual harassment complaints are considered substantiated if they have at least one founded allegation reported in the inquiry or investigation 12 A sexual harassment complaint is considered unsubstantiated if there are no founded allegations as reported in the investigation or inquiry 
military reform efforts should not be based on the current prevalence rates (Rosenthal \& Miller, 2013).

\section{Effects of Military Sexual Trauma}

Military sexual trauma has severe physical, psychological, and social effects that have a significant impact on service members during and after their military service (González-Prats, 2017; Harris, Sumner \& González-Prats, 2018). Physically, victims of MST have higher rates of medical symptoms associated with cardiovascular, pulmonary, neurological, urological, and gynecological conditions (Williams \& Bernstein, 2011) and are at a higher risk for obesity, smoking, heart attacks, and hysterectomies before age 40 than those who have not experienced MST (Katz, Bloor, Cojucar, \& Draper, 2007). Psychologically, MST is strongly connected with post-traumatic stress disorder (PTSD), anxiety, depression and substance abuse for both male and female veterans (Steward, 2013; Stinson, 2013). It has been found that sexual trauma presents a risk for developing PTSD that is as high, or higher, than the risk from exposure to combat (Williams \& Bernstein, 2011) and that male and female MST victims are twice as likely to self-harm and/or attempt suicide as veterans who have not experienced sexual trauma (Kelty, Kleykamp, \& Segal, 2010).

Socially, a survivor's quality of life and ability to maintain and develop personal connections with friends, family, and members of their community are adversely affected by PTSD and the other effects associated with MST, such as diminished occupational functioning and lost wages due to missed work time and unemployment (Suris, Lind, Kashner, Borman, \& Petty, 2004). 


\section{Why is Military Sexual Trauma a Social Problem?}

Sociologist Jerome Manis (1974) defines primary social problems as "influential social conditions which have multiple detrimental consequences for society" (p. 8). Using this definition, I am identifying MST as a collective social problem that has an impact on the military, veteran, and civilian communities alike for three main reasons. Military sexual trauma extends beyond being a military or female problem to a problem that affects all of society (González-Prats, 2017; Harris et al., 2018).

Chief among these reasons is the matter of social responsibility. Service members deserve protection and safety from abuse and assault. MST poses a threat to the values of equality, justice, and dignity because it dehumanizes the service members who have volunteered to protect and defend the country, oftentimes at significant risk and personal sacrifice to themselves and their families (Protect our Defenders, n.d.; Tsongas \& Tardiff, n.d.). MST is a risk that can be prevented and mitigated through consistent implementation of policies of accountability (Caplan, 2013; Halloran, 2013).

A second reason is that the risk of being a victim of MST poses a threat to recruitment and retention, which ultimately affects national security (Vandenbrook \& Jackson, 2013; Tsongas \& Tardiff, n.d.). The military already faces significant recruiting challenges and a shortage of personnel. It is estimated that over $70 \%$ of the target U.S. demographic (17- to 24-year-olds) are ineligible for military service due to obesity, medical conditions, criminality, and education standards (Spoehr \& Handy, 2018). In order to solidify voluntary military service members, ensure proper staffing, and sustain a 
formidable national defense program, it will be essential for the military to ensure safety from sexual victimization from within its own ranks.

Lastly, addressing MST has to do with public safety. Although there is conflicting data about the recidivism and re-offense rates of sexual perpetrators, there is a strong possibility that sexual perpetrators could reoffend upon being released into the general civilian population (Dick, 2012; Horgan, 2013; Military Justice International, 2015). A meta-analysis shows a positive correlation between the increase of recidivism of sexually based offenses with the increase of time, ranging from a rate of $10 \%$ to $15 \%$ after 5 years, to a recidivism rate of $30 \%$ to $40 \%$ after 20 years (Hanson, Morton, \& Harris, 2003).

The public safety concern becomes even greater because not all of the perpetrators who commit sexually based offenses in the military are investigated, courtmartialed, convicted of sexual assault, and/or imprisoned, resulting in an unknown threat in most communities (Dick, 2012; Military Justice International, 2015; Service Women's Action Network, n.d.). Improving military efforts to prevent and respond to MST is important to protect our civilian communities and keep them safe from sexual perpetrators.

\section{What's Missing from the Military's Prevention and Response Efforts}

Despite the progress and reforms in the military's efforts to respond to MST in the areas of the military justice system and victim support services, there still remain four areas that threaten to undermine current efforts and progress made to date, rendering the prevention and response strategies ineffective and unsustainable. 
First, despite the fact that half of MSA survivors are men (O'Brien, Keith, \& Shoemaker, 2015; Steward, 2013), the mandatory MST prevention training required of service members still conveys the female victim/male perpetrator paradigm, thus reinforcing outdated rape myths that only women are vulnerable to sexual violence (CSPAN 2013, 2014; González-Prats, 2017; Hoyt et al., 2011; O’Brien, et al., 2015). Continuing the narrative that only women are victimized harms all service members, as it undermines efforts to effectively address the problem. Additionally, women are best served when the prevalence of MST is understood and addressed beyond the conventional binary of male/female and when the full spectrum of gender identity and sexual preference is included.

Second, when MST prevention training scenarios continue to focus on alcohol and promiscuity (i.e., hookup culture) as root causes of sexual assault instead of the predatory and criminal behavior of perpetrators, the narrative will continue to place sole responsibility for reducing the risk of being sexually harassed or assaulted on the victim.

Third, the military has recently acknowledged in their fiscal year 2016 Annual Sexual Assault Report (DoD, 2017) that service members who identify as LGBTQ are statistically more likely to experience sexual harassment and sexual assault than members who do not identify as LGBTQ. Considering the repeal of Don't Ask, Don't Tell, Don't Pursue ${ }^{13}$ policy in the last 7 years and the current and ongoing controversy regarding

\footnotetext{
${ }^{13}$ A 1993 policy that was passed as a compromise under the Clinton Administration that minimized restrictions against homosexual service members without resorting to an explicit ban on homosexual service members. Military leaders were not allowed to ask about a service member's homosexual orientation which they deemed as "private"; however, acts of homosexual activity/conduct were still against military law, and could be cause for a mandatory discharge from military service. This policy was
} 
transgender individuals serving in the military (Allsep, 2013; De Vogue, 2017; Frank, 2010; Office of the Secretary of Defense, 1993; Rosenberg, 2016), it would behoove the DoD to review MST prevention and response protocols, and leadership training, for exclusively heteronormative ${ }^{14}$ and cisgender ${ }^{15}$ viewpoints (Green \& Maurer, 2015; Herz \& Johansson, 2015), which are not reflective of the totality of servicemember experiences in up today's military.

Lastly, what is missing from the prevention and response efforts is the explicit emphasis that incidents of sexual harassment and sexual assault are interrelated and exist on a "continuum of harm" (DoD, 2014a, p. 21) as found in the 2014 Rand Military Workplace Study, instead of two separate incidents (RAND National Defense Research Institute, 2014). The DoD would benefit from training military supervisors at every level of leadership to treat incidents of sexual harassment as potential sexual assaults, instead of the historical minimization and disbelief survivors experience when reporting incidents of sexual harassment.

The common threads that link these deficient areas are that (a) MST prevention and response strategies and protocols are far removed from the reality of the MST survivor's experience, and (b) the explicit and implicit messaging surrounding the continued incidents of sexual harassment and sexual assault in the military directly contradict the values and principles guiding every soldier, airman, sailor, marine, and

lifted by President Obama in 2010, allowing service members to serve openly, regardless of their sexual orientation.

${ }^{14} \mathrm{~A}$ term that refers to the privileging and normalization of heterosexuality in everyday society.

${ }^{15}$ An adjective to describe a person whose gender identity is congruent with (or "matches") the biological sex they were assigned at birth. (Some people abbreviate this as "cis"). 
coastguardsman. As such, both elements of MST should be studied collectively to truly measure the command climate of a military unit, service branch, and the DoD as a whole as well as determine the effectiveness of MST prevention and response efforts.

\section{Part II: Overview of Study/Purpose}

This section briefly describes the dissertation study, including the goals and purpose as well as its potential value to the fields of social work and social research.

Military sexual trauma is a key and often misunderstood health issue that greatly affects the lives of military personnel and society as a whole (Harris et al., 2018). Military sexual trauma has often been framed by the military community as a gendered violence issue, where, primarily, women are the victims (González-Prats, 2017; Hoyt, Klosterman Rielage, \& Williams, 2011; Steward, 2013). Given the severity and continued prevalence of sexual harassment and sexual assault in the military (Katz et al., 2007; RAND National Defense Research Institute, 2014; Williams \& Bernstein, 2011), the field needs to identify, reconcile, and contend with multiple challenges (GonzálezPrats, 2017).

There have been significant military policy milestones over the 6 years prior to this study allowing gay and transgender service members to serve openly (Allsep, 2013; Frank et al., 2010; Office of the Secretary of Defense, 1993; Rosenberg, 2016) and the unrestricted inclusion of women into all military occupational specialties and direct combat roles (Wire Reporters, 2015). As such, the residual impact of historical policies institutionalizing misogyny and homophobia and its contribution to a culture of discrimination and violence must continue to be part of ongoing research. (Allsep, 2013; 
González-Prats, 2017; Turchick \& Wilson, 2010). The military’s organizational culture of strict accountability, order, and discipline directly contradicts its inability to defeat one of the most recurring and insidious of enemies (González-Prats, 2017). Consequently, MST continues to degrade the collective effectiveness of our nation's military (Vanden Brook \& Jackson, 2013; Tsongas \& Tardiff, n.d.)

The long-term goal of this research is to inform policy and interventions that can better address MST for people of all genders. The objectives of this dissertation study are to identify how current messaging in the military affects the experience of MST survivors and to obtain recommendations to improve prevention and response services. The objectives will be achieved by answering the following three research questions:

1. What was the messaging (via the military's prevention and response efforts) that survivors received about MST from their chain of command and their military unit overall? How did survivors internalize this messaging and how did it impact their experience of MST?

2. What roles (if any) do gender identity and attitudes towards gender play into the military's messaging (e.g. prevention and response efforts) about MST?

3. What (messaging) do survivors feel would have helped? Moving forward, what would survivor-centered prevention and response efforts to MST look like?

\section{Contributions to Field of Social Work and Social Research}

The field of social work has expanded its scope significantly in terms of both practice and scholarship (Social Work Degree Guide, 2017). I foresee my study on MST contributing to two subspecialties within the social work profession: macro social work 
and military social work. As detailed in the next chapter (literature review), much of the research on MST completed in the social sciences sector have focused more on direct clinical practice and the effects of MST than on the macro practice level, which addresses the social problem on a larger scale and examines the stakeholder institutions and systems that serve as either enablers or barriers to interventions and solutions (Social Work Degree Guide, 2017). Secondly, the subspecialty of military social work would benefit from being equally committed to preventing MST systemically as they are to providing more traditional treatment services for survivors and family members. Doing so would help military social workers be more effective advocates and agents of change for institutional reform (Wooten, 2015). 


\section{Chapter Two: Literature Review}

As discussed in the introductory chapter, the existing body of knowledge on MST has focused at the micro/individual level, investigating the physical, psychological, and social effects of sexual assault on victims (González-Prats, 2017; Katz et al., 2007; Steward, 2013; Stinson, 2013) as well as the most effective options for support and treatment.

Conversely, there is a dearth of research at the macro and systemic levels which addresses the continued prevalence (Hulley, 2014) of sexual harassment and sexual assault in the military. There is a need for a critical examination of the military's prevention and response efforts from the vantage point of the most important stakeholder. Providing MST survivors the opportunity to participate in the evaluation process allows them to identify existing gaps between the military's messaging and the reality of their experiences surviving sexual harassment and/or sexual assault.

Given the recent military policies expanding the inclusion of women in combat roles and the continued controversy of transgender individuals serving in the military, the body of MST knowledge would be significantly enhanced if more studies included survivors across the continuums of gender identity and sexual orientation as well as all incidents along the continuum of harm, instead of just focusing on sexual assault. Oftentimes, military service members are treated as a monolith, and identities like gender, sexual orientation, race, and ethnicity are compartmentalized as individual demographics versus studying the role of intersecting identities in incidents of MST. 
This chapter begins by identifying the conceptual framework that influences the literature search in the areas of gender identity and messaging as well as the lens used in the proposed study, drawing from the fields of communications, public health, social psychology, and education. Next, I introduce a discussion of the relevant literature about sexual harassment and sexual assault, drawing from sources within the DoD and congressional testimony from MST survivors. The literature reviewed provides a foundation for the approach and choices of methodology, participant sampling, data collection, and data analysis in Chapter Three.

\section{Conceptual Framework Guiding This Study}

For almost four decades, incidents of sexual harassment and sexual assault have been traditionally framed by the military as an issue of servicewomen's vulnerability and their fitness and suitability to serve as men's equals in defense of the nation.

Consequently, the military's prevention and response efforts have been informed by such narratives (Harris et al., 2018)

The military has resisted repeated calls for reforms to its justice system (Office of Senator Kirsten Gillibrand, 2009) and increased accountability for perpetrators of sexual assault (CSPAN 2013, 2014). Starting with the premise that the military is capable and willing to reverse the trajectory of MST within its own ranks, it is important to critically examine how the messages endorsed in current prevention and response programs are either strategically aligned or incongruent with its stated commitment to eradicate a culture of sexual harassment and sexual assault. 


\section{Intersectionality}

The military has traditionally been centered on the "intersectional identities of a few" (Crenshaw, 1991, p. 1289), privileging White, cisgender, heterosexual men as the heralded warrior standard bearer for all military service members, providing dominant viewpoints (Alford, 2016; Allsep, 2013; Belkin, 2008; González-Prats, 2017).

Consequently, when members of the dominant group serve as the decision makers and organizational stewards (Okros, 2009), the interventions, solutions and policies that ensue will continue to reflect the one-dimensional thinking that represent the dominant point of view (Carbado, 2013; Crenshaw, 1991; Hancock, 2007).

Intersectionality stands out as a powerful framework to investigate MST because it pays attention to the nuances and interconnectivity of identity and power dynamics (Carbado, 2013; Crenshaw, 1991; Hancock, 2007) often minimized or overlooked entirely in seemingly homogenous organization cultures like the military, which place a premium on uniformity and meritocracy. In theory, the military would be devoid of discrimination like sexism, racism, or homophobia. However, the reality is that the U.S. military, like the society it reflects, has an extensive history of policies of institutionalized discrimination to include the segregation of African American and Black servicemen and the exclusion of women and openly gay and transgender service members (Belkin, 2008; González-Prats, 2017; Rostker, 2006).

"Intersectionality" is a term that emerged from Black feminist scholarship and critical legal studies in the 1980s (Cho, Crenshaw, \& McCall, 2013; Hancock 2007), and has been employed broadly and dynamically as an analytical tool as well as a 
theoretical/methodological paradigm. (Cho et al., 2013; Hancock, 2007). Intersectionality "describe[s] the dynamics of differences and solidarities of sameness within the context of antidiscrimination and social justice politics" (Cho et al., 2013, p. 787). Specifically, it pays attention to the concurrent and interdependent roles that a person's identities play in accessing social and political capital, privilege, and an overall sense of equality within their communities and institutions of power (Hancock, 2007).

These identities or "categories of difference" (Hancock, 2007, p. 63) include race, gender, class, and sexual orientation (Carbado, 2013; Cho et al., 2013; Hancock, 2007). There is a prevalence of power, domination, and marginalization within interpersonal violence, sexual harassment, and sexual assault (Crenshaw, 1991; Gorisvlasky, 2014). As such, intersectionality resonated with me as the most dynamic and multidisciplinary framework to investigate and analyze the topic of MST.

Employing an intersectional framework would mean paying attention to the totality of the identities of the survivor and perpetrator and their access, or lack of access, to power (Crenshaw, 1991). Additionally, it would necessitate analyzing

the overlapping structures of subordination, revealing how certain groups [of women $]^{16}$ were made particularly vulnerable to abuse and were also vulnerable to inadequate interventions that failed to take into account the structural dimension of the context. (Cho et al., 2013, p. 797)

\footnotetext{
${ }^{16}$ Original quote included "certain groups of women..." but it could be applicable to other vulnerable groups as well (women, LGBT-Q and /or communities of color, etc.)
} 
It means moving beyond the "colorblind intersectionality" (Carbado, 2013, p.818) where the dominant group/identity speaks for the entirety of the military experience, effectively erasing the experiences of those most vulnerable to marginalization and/or sexual victimization. It also means paying attention to identities such as race and ethnicity in addition to gender and sexual orientation, not as independent or mutually exclusive factors but as dynamic and fluid identities that can be just as empowering or disempowering as that of a military service member (Cho et al., 2013; Hancock, 2007).

\section{Gender Identity Constructs, Sexual Harassment \& Sexual Assault}

For the purpose of this dissertation, I will use feminist theorist Sandra Bem's (1993) lenses of gender as a key construct. This is because the lenses of biological essentialism, androcentrism, and gender polarization (Bem, 1993) effectively anchor the continued prevalence of sexual harassment and sexual assault as well as the singular narratives that explain the etiology of sexual violence in American society:

As profound as the transformation of America's consciousness has been during the past 150 years, hidden assumptions about sex and gender remain embedded in cultural discourses, social institutions, and individual psyches that invisibly and systemically reproduce male power in generation after generation (Bem, 1993, p. 2).

\section{Biological Essentialism}

The justification for social inequality and prejudice has been historically rooted in biological essentialism (Bem, 1993; Morton, Postmes, Haslam, \& Hornsey, 2009), which purports that a person's specific characteristics like masculinity, femininity, intelligence, 
aggression, and passivity are innate, rather than a direct product of their social environment and upbringing (Oxford Reference, 2011). Essentialism has been primarily used to explain the biological differences between men and women. It has also served as a justification for a system where men are at the center of key positions of power, privilege, and social status, known as androcentrism (Abrams, 1993; Bem, 1993; Morton et al., 2009).

\section{Androcentrism}

A male-centered approach goes beyond retaining the power and superiority of men over women. It also includes heralding the male experience as the norm (Bem, 1993). This universal worldview sets up a standard where those with differing viewpoints from the dominant group are othered, and their value or function are always in contrast to the group in power. The most common groups that are othered are women, people of color, and members of the LGBTQ communities as well as and those at the intersection of these multiple identities (Bem, 1993; Crenshaw, 1991). The existence of an androcentric standard creates gender polarization wherein the cultural context of gender always exists within a binary of male/female and a masculine/feminine dichotomy, resulting in strict gender roles that dominate social norms of appearance, emotional expression, and sexuality (Bem, 1993).

\section{Gender Polarization}

Like with androcentrism, gender polarization espouses prescribed scripts for men and women that set up a standardized norm; those who stray from these norms are viewed as deviants, and, consequently, marginalized or excluded from various 
institutions (Bem, 1993). This, in turn, "constructs and naturalizes a gender-polarizing link between the sex of one's body and the character of one's psyche and one's sexuality" (Bem, 1993, p. 81). The viewpoint that gender is a social construct complements the works of Simone De Beauvoir and Judith Butler, both of whom argued that gender is a product of society's expectations and mores, rather than biological certainties (Butler, 1988; Salih, 2002).

In Simone de Beauvoir's critically acclaimed book, The Second Sex, she challenged the construct of gender and sex, stating that "one is not born, but rather, becomes a woman" (Salih, 2002, p. 281). This viewpoint is expanded by the philosopher and gender theorist, Judith Butler (1988) who posited that gender is determined for society by the norms and expectations socially which are constructed from existing institutions and power structures, and, therefore, is highly subjective. Consequently, gender becomes more of a deliberate act of performance, a necessity for survival within an existing social order, than a true identity someone beholds (Butler, 1988). Gender identity becomes more about "something we do rather than something we are" (Salih, 2002, p. 10).

As Butler (1988) explains in her gender as a performance analogy, there are scripts for conventional masculinity and femininity that exist within a strict gender binary that society expects to be followed and performed correctly. Those who deviate from the prescribed scripts are often ostracized and pressured in explicit and implicit ways to return and conform to the conventional social order, or else risk further punitive action: 
that culture so readily punishes or marginalizes those who fail to perform the illusion of gender essentialism should be sign enough that on some level that there is social knowledge that the truth or falsity of gender is only socially compelled and in no sense ontologically necessitated. (Bem, 1993, p. 528)

However, the detail that is often minimized, or left out altogether, in mainstream discussions is that "gender" was a social constructed term created in the 1970s to simplify and expand biological terminology so as to be understood by a non-scientific person. As such, gender is not a concept that can be accurately and interchangeably used with the word "sex," although it often is, much to the detriment of advancing discourse on gender as a social construct (Krieger, 2003). The ongoing debate became a classic chicken or egg argument about the differences between men and women regarding what was ultimately responsible for the observed social roles, interaction and performance between men and women and whether it was due to the innate biological differences between the sexes or the existing social conventions and mores (Krieger, 2003).

Examples of challenges to these socially constructed forms of gender identity are present in everyday life and can be as explicit as the presence of women in traditionally male occupations, especially jobs that require physical strength such as the military, law enforcement, trucking, and the trades industry. Additionally, more implicit and innocuous examples include single parenting by both women and men, dual-career households, and women being primary breadwinners. 
The Intersection of Gender, Sexual Harassment, and Sexual Assault

The lenses of gender played significant roles in establishing, sustaining, and normalizing men as the cultural standard bearers of power, privilege, and status (Bem, 1993), which is consistent with the elements of patriarchy "where men as a group dominate women as a group, both structurally and ideologically" (Hunnicutt, 2009, p. 557).

A consequent outcome of sexual inequality coexisting with strict gender roles is the continued prevalence of violence against women (Bem, 1993) and a normalization of male sexual aggression and perpetuation of a rape culture wherein social and cultural practices include victim-blaming, slut-shaming, and minimization or denial of rape (Meier \& Medjesky, 2018; Sills et al., 2016).

The normalization of sexual violence and the influences of the gender lenses (Bem, 1993) can be viewed within the cultural etiology of sexual violence, specifically within ambivalent sexism (Glick \& Fiske, 1997) and rape myth acceptance (Burt, 1980). Acts of gender-based discrimination such as sexual harassment and sexual assault are based on what psychologists Glick and Fiske (1997) describe as ambivalent sexism. Consisting of two complementary components of behaviors, benevolent and hostile sexism, ambivalent sexism is dependent on (a) overtly marginalizing women, (b) maintaining traditional gender roles, and (c) reinforcing a power differential. Since acts of benevolent sexism include protective and deferential treatment of women (such as chivalry), benevolent sexism seems like a more passive and innocuous form of sexism 
and gender discrimination, which, consequently, can be overlooked and even be deemed socially acceptable (Glick \& Fiske, 1996, 1997; Yamawaki, 2007).

Conversely, hostile sexism includes more overt acts of exploitation, hostility, and demeaning of women that includes objectification, harassment, and sexual violence (Glick \& Fiske, 1996, 2007; Yamawaki, 2007). According to Glick and Fiske (1997), Hostile and benevolent sexism may differ in the valence they place on the attitude object "women", but they share common assumptions (e.g., that women are the weaker sex): both presume traditional gender roles, and both serve to justify and maintain patriarchal social structures. (p. 121)

As with ambivalent sexism, an endorsement of traditional gender roles and behaviors facilitates an acceptance of rape myths, which are described as the stereotypical beliefs which often minimize and excuse the acts and perpetrators of sexual violence while concurrently placing the blame and responsibility on the victim for prevention (Carroll, Fouber, Rosenstein, Clark, \& Korenman, 2016; Chapleau, Oswald, \& Russell, 2008; Suarez \& Gadalla, 2010; Yamawaki, 2007).

\section{Ambivalent Sexism and Rape Myth Acceptance: A Review of the Literature}

There are five papers with empirical data that discuss the interdependent roles that ambivalent sexism and rape myth acceptance (RMA) play in the prevalence of sexual violence (Abrams, Viki, Masser, \& Bohner, 2003; Carroll et al., 2016; Chapleau et al., 2008; Suarez \& Gadalla, 2010; Yamawaki, 2007).

The first paper authored by Abrams et al. (2003) consisted of four experiments that investigated victim-blame and the contributions of benevolent and hostile sexism in 
men's rape proclivity within the context of stranger versus acquaintance rape. A participant sample of 65 students was drawn from a mid-size university in England ${ }^{17}$. The study series also employed a combination of male/female participant groups and maleonly participant groups to identify viewpoints on gender roles and rape proclivity. The researchers randomly assigned participants to read different rape scenarios and assessed their views on hostile and benevolent sexism as well as their acceptance of rape myths using multiple measures.

There were two key findings in this paper. First, victim-blaming was associated with higher rates of benevolent sexism, more so in cases of acquaintance rape than of stranger rape, citing the victim's behavior as inappropriate or problematic and perhaps inviting the violence. Second, there was a relationship found between a rate of higher hostile sexism and an increased rape proclivity towards acquaintance rape. Abrams et al. (2003) found that, after controlling for benevolent and hostile sexism, RMA was a significant predictor of victim-blaming and rape proclivity. The investigators concluded that solely relying on RMA score to collect data about victim-blaming and rape proclivity would be too limiting to capture the differences in attitudes towards acquaintance versus stranger rape. Instead, Abrams et al. proposed continuing to examine the relationship between both types of sexism and the attitudes towards potential victims and perpetrators to gauge their influence on cultural norms.

17 Abrams et al. (2010) demographics included: 52\% women, $80 \%$ European with $85 \%$ of the sample being 26 years old and under 
The second paper built on the contributions of strict gender role adherence to sexual violence and victim-blaming. Using a sample of 126 U.S. undergraduate students $^{18}$, Yamawaki (2007) explored the roles that benevolent sexism, hostile sexism, and gender role traditionality play in the secondary victimization of rape victims. Similar to Abrams et al. (2003), Yamawaki randomly assigned the study participants to read either an acquaintance rape or stranger rape scenario and surveyed their views using multiple measures.

There were three main findings that provided insight regarding the degree to which participants would blame the victim and/or excuse the perpetrator's behavior. First, the researcher found that the respondents minimized the seriousness of rape more so in the date rape, or acquaintance rape, scenarios as compared to the stranger rape scenarios. Hostile sexists tend to view women's reactions to rape as hyper-sensitive and exaggerated. Second, Yamawaki (2007) found that benevolent sexism and gender role traditionality were significant predictors of victim-blaming in the date rape scenarios, but not in the stranger rape scenarios. Third, hostile sexism was the only significant predictor in excusing the perpetrator, as hostile sexists tend to espouse the beliefs that women use their sexuality to gain special treatment and take power away from men. An adherence to strict gender role traditionality played a significant role in whether women were believed or blamed for their own sexual victimization. Those who espouse gender role traditionality believe that the only women who can benefit from protected and deferential

18 Yamawaki (2007) study demographics included: 46\% women, 89\% White/Caucasian and the average age was 20 years old. 
status are those who follow their assigned roles. In contrast, women who adopt the norms that are more socially acceptable for men, such as alcohol abuse, and abuse, and promiscuity tend to be less protected or outright punished (Yamawaki, 2007).

In the third paper, Suarez and Gadalla (2010) conducted a meta-analysis ${ }^{19}$ of 37 studies $^{20}$ to update North American literature on the association of RMA with demographic, behavioral, and attitudinal variables as well as the strength and direction of these associations. Demographically, ${ }^{21}$ the investigators found gender had the strongest relationship with RMA; men showed higher rates than women. In the male-only participant samples, masculine playboy and athlete ideals were positively associated with a higher RMA rate. Whereas higher levels of RMA were endorsed by individuals with lower education levels, age was not a statistically significant factor.

Suarez and Gadalla (2010) found a positive correlation between RMA and multiple other oppressive worldviews, which include sexism, misogyny, victim-blaming, and the acceptance of interpersonal violence. Conversely, positive racial identity was a protective factor associated with lower rates of RMA. This particular study's findings were especially significant because they explicitly addressed the positive correlation between RMA and other oppressive behaviors such as racism, heterosexism ${ }^{22}$, classism, ageism, and religious intolerance, making it a broader socio-cultural issue that would

19 Suarez \& Gadalla (2010) meta-analysis of 37 studies includes 34 studies from the U.S. and 3 from Canada

20 Suarez \& Gadalla (2010) meta-analysis demographics included a total of 11,487 participants 21 Suarez \& Gadalla (2010) participant demographics included 58\% men, averaged 85\% White and the average age was 25 years old

22 Suarez and Gadalla (2010): only four of the 37 studies (11\%) reported the sexual orientation of participants 
benefit from specific prevention programming addressing these areas: "The significant moderating role of high racial identity and openness to other races and cultures, or multiculturalism also indicate areas to focus on in programs targeted to reduce RMA" (Suarez \& Gadalla, 2010, p. 2027).

The fourth paper by Chapleau et al. (2008) was unique in that it specifically focused on exploring the ideologies that facilitate male rape myths and ambivalent sexism toward men, focused on three of the most prevalent rape myths: male rape does not happen, rape is the victim's fault, and men would not be traumatized by rape

The investigators used a total participant sample of 603 college students ${ }^{23}$ both from a small public college ${ }^{24}$ and a mid-sized religious university. ${ }^{25}$ This study surveyed the mixed-gender sample using the male rape myth scale (Struckman-Johnson \& Struckman and Johnson, 1992), the female RMA scale (Payne, 1999), the adversarial sexual beliefs and acceptance of interpersonal violence measures(Burt, 1980), and the ambivalence toward men inventory (Glick \& Fiske, 1999). There were four key findings that provided insight into attitudinal differences of attitudes toward the acceptance of male rape myths.

The investigators found that men were more accepting of male rape myths than women, regardless of whether the victims were women or men, which was consistent with Suarez and Gadalla's (2010) findings. However, there was less of a difference

23 Chapleau et al. (2008) combined sample was $65 \%$ female, $85.2 \%$ White/Caucasian and with a mean age of 19.6 years old.

24 Chapleau et al. (2008) Catholic university sample (57\%: $n=246)$

25 Chapleau et al. (2008) Catholic university sample $(n=180)$ 
between the participant responses regarding the endorsement of male rape myths when the perpetrator was a woman as compared to when the perpetrator was a man. The strongest predictor for male RMA was the concurrent acceptance of interpersonal violence for both male and female participants. Additionally, benevolent sexism towards men, such as that men are supposed to be physically and sexually invulnerable, is associated with male RMA. Consistent with the findings of Abrams et al. (2003), Chapleau and colleagues (2008) found that it would be more advantageous to disaggregate the male myth scale by type (denial, blame, trauma), especially within the gender variable to identify the nuances of attitudes towards victims, perpetrators, and rape, rather than solely relying on the overall RMA score.

The final paper, authored by Carroll et al. (2016) assessed the pattern of RMA among the 18- to-24-year-old demographic within the university setting of two unique cultural groups, college sorority and fraternity members from a large public university ${ }^{26}$ and cadets ${ }^{27}$ and midshipmen ${ }^{28}$ attending military service academies. Carroll and colleagues (2016) chose the sample because of the selective nature of membership and the values such as hyper-masculinity, strict gender roles and male dominance that these organizations espouse and which are associated with a rape-supportive culture. The researchers identified three key findings.

26 Carroll, Fouber, Rosenstein, Clark \& Korenman (2016) study included 393 participants (48\% men; 90\% White; mean age is 18)

27 Carroll, Fouber, Rosenstein, Clark \& Korenman (2016) study included 1,169 cadets (85\% men, less than $20 \%$ identify as non-White or other, mean age is 18 ) from the United States Military Academy, also referred to as "West Point".

28 Carroll, Fouber, Rosenstein, Clark \& Korenman (2016) study included 1,916 midshipmen $(81 \%$ men, nearly $30 \%$ identify as non-White or other, mean age is 18 ) from the United States Naval Academy 
First, there were three rape myths most frequently endorsed by the participants that deny male perpetrator culpability, support ideas that drinking and attire worn were partially responsible for the violence, and reinforce the belief that women lie about being raped.

Second, consistent with other RMA study findings (Chapleau et al., 2008; Suarez \& Gadalla, 2010), women showed lower levels of RMA than men, including a greater amount of variability of levels among military service academy and fraternity men than their female counterparts. Third, Carroll et al. (2016) found two areas within the sample that differed: (a) male and female military service academy members were more closely aligned in views towards rape, and (b) men and women were similar in ranking rape myths but there was a higher RMA found in the Navy than in the Army.

The above findings within the ambivalent sexism and RMA literature underscore several areas of commonality as well as reveal gaps that need to be addressed by future studies. There are three significant implications for current and future sexual assault prevention and response efforts. First, the existing empirical literature is predominantly based on examining sexual harassment and sexual assault within the male/female binary and within a heteronormative framework with an emphasis on the female victim/male perpetrator paradigm of sexual violence (Abrams et al., 2003; Carroll et al., 2016;

Chapleau et al., 2008; Suarez \& Gadalla, 2010; Yamawaki, 2007).

Second, more than $80 \%$ of the participants in these studies identified as White, which results in limited representation of ethno-racial groups, especially the intersecting 
roles various forms of oppression like racism, homophobia, and religious intolerance play in the prevalence of sexual violence (Suarez \& Gadalla, 2010).

Last, research needs to move beyond solely relying on applying aggregated scores from ambivalent sexism inventory and RMA scales uniformly across all academic and workplace settings. Instead, by paying attention to specific organizational cultures and adopting more intra-scale analyses to explore the nuance of attitudes towards victims and perpetrators, investigators can help identify additional confounding variables and predictors for further study: "Understanding cultural characteristics of organizations and institutions that may foster different rape myths for men and women can help us come closer to identifying targeted educational efforts to address false beliefs about rape" (Carroll et al., 2016, p. 315).

\section{Messaging, Sexual Harassment, and Sexual Assault}

The most problematic areas of society, depending how they are framed, significantly influence how individuals and communities think about the problem as well as how they conceptualize potential solutions (Mejia, Somji, Nixon, Dorfman, \& Quintero, 2015). An important cultural cornerstone of information is how it is communicated, which is just as important as the actual context of the information. A concept known as framing, this process selectively highlights specific aspects and issues to promote a certain interpretation, which, depending on how it is characterized, can wield a significant amount of influence, helping the audience understand the issue while signaling which topics are important for audiences to think about (Entman, 2002;

Scheufele \& Tewksbury, 2007). Besides communicating a certain viewpoint, or solution, 
"frames are tools that help explain complex issues and make them more accessible to audiences" (Scheufele \& Tewksbury, 2007, p. 12).

Using the analogy of a picture frame, the types of views can best be explained within a portrait versus landscape context. Whereas a portrait view focuses on an individual person or event, a landscape view offers a more detailed and comprehensive view that includes more of the surrounding environment affecting the person or event (Berkeley Media Studies Group, 2018b). Traditionally, the public tends to be exposed to news stories that focus on the portrait view, thus providing a singular aspect of an event or issue, which can limit identifying contributing factors and potential solutions (Berkeley Media Studies Group, 2018b). “The selection -- or omission--of arguments and voices functions similar to a frame around a photograph, telling us what information is important, and what information we can ignore" (Berkeley Media Studies Group, 2018b, p. 1).

Incidents of sexual harassment and sexual assault are most often communicated to the public via traditional news coverage such as television and print media ${ }^{29}$ and are usually framed as criminal justice issues. With a focus on individual responsibility for preventing or responding to the incident, and through a limited portrait lens, what is minimized or altogether absent are factors from social and cultural institutions which contribute to mitigating risk (Mejia et al., 2015). As a result, prevention and response

29Although it is beyond the scope of this dissertation, it is important to acknowledge that more than $67 \%$ of Americans report receiving their news from social media sources, which includes Facebook and Twitter (Shearer \& Gottfried, 2017) 
strategies are also missing (Bell, Stein, \& Hurley, 2017; Garcias-Rojas, 2012; Mejia et al., 2015).

\section{The Framing of Sexual Violence in our Culture}

In this section, I review the scholarship on communication and messaging, specifically how sexual violence is framed in our culture, as well as how the frames influence prevention and response efforts. According to the National Sexual Violence Center (2010), acts of sexual harassment and sexual assault exist within a continuum of sexual violence which includes rape, incest, child sexual abuse, intimate partner violence, sexual exploitation, human trafficking, unwanted sexual contact, exposure, and voyeurism (Mejia et al., 2015; National Sexual Violence Resource Center, 2010).

There are two studies in the empirical literature that highlight how sexual harassment and sexual assault are communicated to the public as well as how people view the incidents, the extent of the prevalence, and who is responsible for prevention. (Mejia et al., 2015; O’Neil \& Morgan, 2010). In the first study, the National Sexual Violence Center and the Berkeley Media Studies Group collaborated to examine the framing of sexual violence in the media and how it influences nationwide prevention efforts. In addition to conducting a content analysis of news coverage from 2011 to 2013, the investigators also conferred with advocates about the public narratives on sexual violence and prevention efforts in the United States. Three key findings revealed how sexual violence was framed by U.S. news coverage and implications for national prevention efforts (Mejia et al., 2015). 
First, Mejia and colleagues (2015) found that incidents of sexual violence are often treated as independent and unrelated offenses and, as such, are selectively reported in news coverage. A majority of the reported incidents are of the rape and sexual assault of older teens or adults and child sexual abuse and are usually the most extreme or sensational types of cases. Conversely, what is minimally represented or absent altogether are incidents of sexual harassment, sex trafficking, and child sexual exploitation. News stories promote a skewed narrative of who experiences and who commits sexual violence and is usually focused on female victims. Not surprisingly, men who have experienced sexual violence have pointed out that the news coverage minimizes and/or inaccurately reports their experiences (Mejia et al., 2015).

Second, Mejia and colleagues (2015) noted that the issue of sexual violence is usually portrayed within a criminal justice context, instead of a public health or social justice issue. This, in turn, affects what the news focuses on. The focus on criminal justice-related events and subject matter expertise tends to be drawn from criminal justice representatives like law enforcement, whereas survivors and sexual prevention advocates are infrequently consulted about direct experiences and viewpoints about sexual violence. Additionally, the role of institutions in addressing sexual violence is more often focused on the criminal justice system versus others like universities, churches, schools, and the military, which also play significant roles in prevention and response efforts.

Third, the researchers found that news coverage is rarely focused on prevention or solution efforts. Whereas services for those accused, like therapy, or settlements for victims in civil cases are rarely mentioned, more than $54 \%$ of the articles in the study 
focused on consequences for the person accused, such as loss of job, arrest, or detainment. In the rare event that solutions are discussed, they are focused on riskreduction, where the responsibility is on the victim to prevent the violence, or bystander intervention, where nearby individuals are expected to intercede and prevent the violence from occurring. Additionally, the narrow framing of the social problem from a dominant perspective guarantees one-dimensional solutions and responses as well as an absence of intersectionality, which masks the true prevalence of the sexual victimization, especially people of color, men, and LGBTQ individuals, who are often absent from consideration.

The second study, conducted by O’Neil and Morgan (2010), used social discourse analysis to define sexual violence, its causes, and how it may be addressed through policies and interventions. The researchers interviewed 30 practitioners, sexual assault prevention subject matter experts, and individuals from the general public. The members of the general public were classified as informants in this study. There were three key findings that provided insight into the dominant cultural model of sexual violence as well as the gaps in understanding between subject matter experts and laymen and areas for future research.

First, sexual violence was defined by the experts as a continuum of behaviors that extends beyond physical acts and occurs amongst people who know each other, despite the news coverage portraying violence from strangers and it being the least common form of occurrence. The experts felt that the focus on sexual violence solely as a woman's issue compromised the public's understanding of their role in preventing it. In contrast, the informants classified sexual violence as acts completed and without consent. 
Attempts and threats of violence were not defined as sexual violence, and victimization was attributed to vulnerability and traits usually associated with women and young children.

Second, the subject matter experts in O'Neil and Morgan's (2010) study traced the causality of sexual violence to the culture of unequal power relationships in terms of race, gender, socioeconomic status, and other factors where even the existence of sexist language and attitudes reinforces a culture of inequality. On the other hand, informants attributed the risk of sexual violence to conflicting etiology: (a) random occurrences and (b) acts that specifically occur in urban environments, especially at night and are more a product of poor upbringing than of poverty or limited resources. Although informants did not explicitly subscribe to victim-blaming, there was a dominant view that individuals were responsible for their situational awareness and taking the necessary precautions to protect themselves. This underscored an important point that further prevention efforts need to move beyond framing sexual violence prevention within an individual context that focuses the blame and responsibility on victims.

Third, the informant sample viewed the rehabilitation of predatory sexual behavior as impossible. Consequently, they felt that that sexual violence prevention and deterrence efforts should be focused on the physical separation of victim and perpetrator. The suggestions for deterrence ranged from tougher prison sentences to male castration as a mean of preventing repeat offenses. In contrast, the subject matter experts reasserted that sexual violence is a preventable social issue versus an inevitability of day-to-day life. Additionally, most of the experts believed that prevention efforts must be focused on 
changing cultural norms regarding gender inequality and that any future interventions need to be evidence-based and rooted in research and evaluation.

The multiple models in O'Neil and Morgan's (2010) study reveal ways to look at sexual violence which underscore the contradictions and nuances that are not often present in dominant discourse, news coverage, and prevention/intervention efforts. The researchers identified a need to provide other models about sexual violence that extend beyond the good/bad binary of predatory behavior and include broader concepts of consent and harm that help draw attention to acts that are not seen as sexual violence, such as lewd comments or harassing behavior. As such, "the cognitively opportunistic of application of one or the other in a set of conflicting models is key to understanding the widely varying views and opinions that Americans have about sexual violence and designing strategic communications” (O’Neil \& Morgan, 2010, p. 12).

\section{The Foci of Sexual Violence Prevention Programming}

One of the major challenges that victims of sexual harassment and assault face is that they have to justify their victimization and prove they were not responsible for the incident, as opposed to victims of other crimes (Holstein \& Miller, 1990; Venema, 2016). Consequently, the majority of sexual harassment and sexual assault prevention programs are concentrated in two primary areas: risk-reduction and bystander intervention (Hertzog \& Yeilding, 2009; Mejia et al., 2015). These areas are the most visible and utilized in the workplace and higher education settings (Harned, Ormerod, Palmieri, Collingsworth, \& Reed, 2002; Hertzog \& Yeilding, 2009; Potter, 2010). Whereas risk-reduction programs are focused on individual measures to decrease the chances of being victimized, 
bystander intervention programs are aimed at educating the general public about warning signs and ways to intervene in a potential harassment or sexual assault situation. The common threads that runs through both these approaches is that (a) the responsibility for prevention rests on everyone but the perpetrator, and (b) the framing of sexual violence is treated as an inevitability, instead of as a preventable issue.

As Mejia and colleagues (2015) found, the majority of prevention programs concentrate on communicating risk-reduction or avoidance tips for individuals, usually aimed at young women, and primarily focus on avoiding victimization by self-monitoring intake of alcohol and recreational drugs as well as keeping track of their drinks at parties to prevent the ingestion of incapacitating drugs, such as rohypnol, otherwise known as roofies or date rape drugs, which have been linked to incidents of sexual assault, especially on college campuses (Hertzog \& Yeilding, 2009).

On the other hand, the focus of bystander intervention programs shifts the public's mentality about sexual violence prevention being an individual responsibility to it being a collective societal responsibility. These programs are traditionally aimed at the general college student population as well as those identified as at high risk for victimization and perpetration, such as student leaders and members of sororities and fraternities (McMahon, 2010). There are two studies that highlight the effectiveness of bystander interventions, as well as areas of opportunity for future research.

First, Coker, Fisher, Bush, Swan, Williams, Clear, and Degue (2015) conducted a comparative study to evaluate the impact of the Green Dot Bystander program on the interpersonal violence rates of undergraduate students on three college campuses, one 
with the program and the other two without it. Green Dot programs consist of two components: outreach speeches in introductory undergraduate classes and a 6-hour intervention training. The investigators found that, on the campus implementing the Green Dot program, there were significantly lower rates for sexual harassment and stalking victimization (11\%), perpetration (19\%) and overall violent victimization (9\%), as compared to the two comparison campuses without the program.

The improved rates of victimization on the intervention campus suggests that the effectiveness of the Green Dot program stretches beyond individual training to the community level. However, Coker and colleagues (2015) advocated moving beyond cross-sectional studies and contributing to the empirical body of knowledge through longitudinal studies. Critically examining the effectiveness of intervention programs like Green Dot would provide richer data on changed bystander behaviors, violent victimization, and perpetration patterns (Coker et al., 2015).

The second study conducted by Rich, Utley, Janke, and Moldoveanu (2010) surveyed a diverse pool (65\% non-White) of male college students for their perspectives on prevention education for violence against women. These investigators intentionally chose this specific male-only sample because they hypothesized that the participants would believe that the topic of violence against women would be irrelevant to them or that the problem is greatly exaggerated by women, would most likely agree with rape myths, would become defensive towards prevention education, and that learning about gender and violence against women would make them seem less masculine or more like a homosexual. 
There were multiple findings that revealed a great deal about the sustainability of gender-specific programming in prevention education. First, more than half of the participants were opposed to attending a mandatory prevention education program because the topic seemed irrelevant to them, which closely mirrored the viewpoint shared by $63 \%$ of the sample who did not want to attend because they seemed unaware of the role men played in promoting rape culture and that violence against women were acts perpetrated by other men. Next, 19\% directed the blame at women for their own victimization and "were quick to offer advice on ways to avoid rape while simultaneously invoking rape myths about manipulative women, suggestive clothing and provocative behavior" (Rich et al., 2010, p. 274).

Rich et al. (2010) found a multitude of responses about the male's role of responsibility in preventing sexual violence. The responses ranged from relying on more traditional gender stereotypes like chivalry and physical intervention during an assault or violently retaliating against a perpetrator $(21 \%)$, to increasing awareness by attending a class or early intervention education. The most unexpected finding came from the response of $85 \%$ of the participants who viewed the prospect of male peer educators favorably. The researchers were surprised by this finding because it contradicted their earlier hypothesis that male attendees at violence prevention programs would be viewed by their brethren as less masculine and their sexuality would be questioned.

Lastly, although many participants shared feelings of trepidation about being in prevention education classes with women because of potential discomfort and conflict, nearly $80 \%$ of the respondents believed a mixed-sex audience would lead to a more 
dynamic, robust, and collaborative effort to fight sexual violence. Rich et al. (2010) concluded that, in addition to "continued efforts to recruit diverse subject pools and consider racial, ethnic and cultural implications of prevention education" (p. 284), there is an area of opportunity for prevention education programs to engage men in such a way that transcends traditional gender roles while remaining cognizant of potential defensiveness.

\section{Recommended Communication Strategies for Sexual Violence Prevention}

There have been multiple communication strategies recommended by researchers that build on the findings of the gender identity, sexual violence and messaging literature discussed earlier in the literature review (Centers for Disease Control and Prevention [CDC], 2015; Dahlberg \& Krug, 2002; Gusfield, 1981; Mejia et al., 2015; National Sexual Violence Resource Center, 2010; Potter, 2016). These communication strategies, in turn, significantly shaped my study in the three key areas discussed in the following sections.

\section{Defining Sexual Violence}

Consistent with the findings by Mejia and colleagues (2015), how an issue or problem is defined, by whom, and how it is framed strongly influence the direction and focus of the problem-solving. As such, acts of sexual harassment and sexual definition need to be explicitly defined within a more comprehensive context of the sexual violence continuum which includes rape, incest, child sexual abuse, intimate partner violence, sexual exploitation, human trafficking, unwanted sexual contact, exposure, and voyeurism (Mejia et al., 2015; National Sexual Violence Resource Center, 2010). Doing 
so would help provide a landscape view of the issue of sexual violence, which would help advocates, policy makers, and other stakeholders be more strategic about prevention strategies as well as identify potential connectivity between various forms of violence.

\section{Public Health Approach to Sexual Violence}

Instead of framing the continuum of sexual violence as a criminal justice issue, researchers recommend addressing it as a public health issue that affects the safety and well-being of the entire population (Dahlberg \& Krug, 2002). Public health approaches pay attention to the contributions of individual, relationship, social, and cultural factors in the continued prevalence of sexual violence to maximize the benefits for the largest number of people, according to the World Report on Violence and Health (Dahlberg \& Krug, 2002), and the CDC (2015). The public health field acknowledges that prevention is a dynamic and fluid process. Public health draws from the expertise and engagement of public and private stakeholders, as well as from a multitude of disciplines, including criminology, epidemiology, sociology, education, and economics (CDC, 2015).

As with conventional infectious diseases and illnesses, public health strategies towards violence are grounded in the theory that violence is preventable and favor a fourstep scientific model. This process balances the importance of (a) establishing the magnitude of the problem and (b) identifying the risk and protective factors of becoming a victim or perpetrator of violence with the need to use (c) evidence-based approaches to planning and evaluation efforts to determine program effectiveness, and the (d) overall dissemination at the local, national, and global levels (CDC, 2015). Regardless of the size 
and scope, the public health field favors a formal evaluation of all prevention efforts, especially local programs initiated at the community levels (Dahlberg \& Krug, 2002).

Most of the developed countries focus on the secondary (i.e., immediate response to violence like medical treatment for rape victims) and tertiary (i.e., long-term care responses that are trauma and disability-related) approaches to prevention (Dahlberg \& Krug, 2002). The World Health Organization recognized the significant gaps in public health strategies and consequent need to invest more resources at the primary level of prevention, which would prevent violence before it occurs (Dahlberg \& Krug, 2002). However, one of the classic conundrums of committing and sustaining an increased level of resources for primary prevention efforts is the overall dearth of research and positive examples of tangible and successful violence-free program outcomes to follow or replicate in communities, thus promising a return on investment (Dahlberg \& Krug, 2002):

Framing the news about sexual violence in the context of prevention can help shift the public's and policymaker' perception of sexual violence from a sense of risky, random inevitabilities to a focus on specific rates, causes of violence and prevention strategies. (Dahlberg \& Krug, 2002, p. 20)

As Mejia and colleagues (2015) pointed out, engaging more prevention advocates and survivors in the problem-solving and sexual violence prevention-planning stages would significantly shift the frame from a criminal justice, or portrait, view towards a public health, or landscape view which balances a report of consequences for sexual assault perpetrators with the resiliency of survivors and improved public policy. 


\section{Applying the Lessons Learned from the Anti-Drunk Driving Movement to Sexual Violence Prevention}

There are multiple lessons that can be gleaned from the issue of drunk driving in the 1980s and the shift in public conscience from a private issue and socially acceptable behavior towards an increased sense of collective responsibility in prevention, resulting in significant policy changes (Gusfield, 1981; Potter, 2016). Prior to the 1980s, the responsibility to gauge a person's level of intoxication and their ability to drive was left to the individual to determine, with limited to no involvement or assigned responsibility or ownership from the general public for prevention of the resulting accidents, injuries, and deaths. As a result, prevention responses remained entrenched solely with consequences, as exemplified by drunk driving laws, rather than focusing on causes and had limited to no representation of other stakeholders in the conversation for determining causality and policy. Gusfield (1981) asserted that this divide of "private behavior and public authority" (p. 16) was dependent on a polarization of assigning responsibility and ownership of defining or solving the problem in two ways: causally or politically. While causal responsibility purports that there are a sequelae of factors that explain the existence of the problem, political responsibility "asserts that somebody/some office is obligated to do something about the problem, to eradicate or alleviate the harmful situation" (Gusfield, 1981, p.11).

Potter (2016) focused on comparing and contrasting the anti-drunk driving movement of the 1980s to the current sexual assault prevention efforts on college campuses throughout the United States. The drunk driving prevention model was used to 
illustrate how the evolving views toward drunk driving significantly influenced a change in law and policy, resulting in decreasing alcohol-related traffic fatalities from $60 \%$ in the mid-1970s to $31 \%$ in 2012 .

Prior to the 1980 s movement, drunk drivers were not seen as criminals; they were viewed more benevolently as addicts or upstanding individuals who exercised poor judgement. Both viewpoints garnered public sympathy and understanding, which was exemplified by the higher blood alcohol content threshold and minimal legal fines or penalties that were enforced. One of the most significant catalysts in shifting public opinion was the rise in advocates, many of whom lost family members to drunk driving. Change agents like Remove Intoxicated Drivers in the late 1970s, Mothers Against Drunk Driving, and Students Against Drunk Driving in the 1980s provided an approach more centered on the experiences of the victims and surviving family members, rather than the drivers. What ensued were various public service announcements that communicated strategies for intervening with friends and family members too drunk to drive and engaged a multitude of stakeholders beyond law enforcement like owners of bars and other establishments that sell alcohol. Consequently, a significant shift in public opinion occurred, resulting in viewing the prevention of drunk driving as a collective and social responsibility, which eventually led to stiffer criminal fines and penalties for perpetrators.

When comparing collegiate sexual assault prevention efforts and the anti-drunk driving movement, Potter (2016) identified several lessons learned that provide a concrete roadmap that can be applied to sexual assault prevention moving forward. First 
and foremost, campus policies need to focus on victims/survivors. Second, bystander interventions and public service announcements are only as effective as bystanders feel supported by peers to prevent sexual violence. Last, Potter used the socio-ecological model (Bronfenbrenner, 1977) as a conceptual framework to approach strategies for more sustainable social change, positing that "desired individual and group behaviors are promoted and supported by higher level-actions and policies" (Potter, 2016, p. 826) that focus beyond the default individual level in terms of support and services for the victim or punitive action for the perpetrators and, instead, engage multiple levels: relational (e.g., sexual assault prevention education); community (e.g., policies that limit alcohol on campus); institutional (e.g., enforcement of Title IX legislation); and societal (e.g., use of social media challenging/supporting rape myths).

\section{Gender Identity and Messaging in the Military}

There are several areas of the sexual violence and prevention literature in the civilian sector that provided a helpful roadmap for the DoD to address the continued prevalence of sexual harassment and sexual assault, as well as ways to craft communication strategies that are survivor -focused and adopt a public health approach to problem-solving.

\section{Military Description and Response}

Incidents of sexual harassment and sexual assault have been traditionally framed by the military as issues that (a) are two separate and unrelated acts, (b) affect young and lower enlisted women, (c) occurs after duty hours, (d) occur at social gatherings where alcohol is served, and (e) are a direct result of a lapse of judgement on behalf of the 
perpetrator or a misunderstanding between the victim and perpetrator (Baldor, 2018; Clift, 2013; DoD, 2014; RAND National Defense Research Institute, 2014). Consequently, this narrow framing has dominated the discourse about sexual harassment and sexual assault and heavily influenced the military's prevention and response efforts.

In this section, the issue of MST is examined within the context of gender identity and messaging. I identify how the military describes the issue through its formal reports along with subsequent prevention and response efforts. I also present the gaps between the military's response and the MST survivors' experience.

What is missing from the larger narrative is the reality of the non-stop operational environment that is the military workplace and its recent inclusion of women and LGBTQ service members, over the 5 years prior to this study, allowing them to serve in the unrestricted capacity afforded their male heterosexual cisgender male peers (Gurung et al., 2017).

\section{Department of Defense Military Sexual Trauma Reports}

Although the military has been tracking incidents of sexual assault since 1988, there was not a coordinated DoD-wide effort to address sexual violence until 2005 when the Sexual Assault Prevention and Response Office (SAPRO) was created as a "single point of accountability" (DoD, 2014). in the reports which underscored the need for a continued modernization of the prevention and response efforts from 2005, when the Sexual Assault Prevention Response Office was formally created.

There have been several reports, consisting of surveys and focus groups across all branches of service and military academies, including both the active duty and 
reserve/National Guard components since 2014 that have contributed to MST scholarship as well as issues of gender discrimination and relations (DMDC, 2014, 2016; DoD, 2014; RAND National Defense Research Institute, 2014; Namrow, Hurley, Cook, Van Winkle, \& Hurley, 2016; Namrow, Hurley, Van Winkle, \& De Silva, 2016). There were three themes present within the triad of a sexual assault prevention strategy: victim support services, training, and the military justice system.

\section{Victim Services and Support}

First, the most consistent finding across the various surveys and focus groups is that there has been a noticeable improvement to services and support to victims of MSA (DMDC, 2014, 2016). The most popular enhancement has been the 2011 creation and implementation of the Special Victims Counsel and the Victim's Legal Counsel, which serve as guides and advocates for sexual assault victims during the military justice process (Namrow et al., 2016a, 2016b).

Another resource that has been helpful for victims is the change in expedited transfers policy allowing a unit commander discretion to transfer either the victim or perpetrator (DMDC, 2014). On one hand, there is support for this service because it would help keep the victim safe, either in their original unit or in a new one, separated from the perpetrator. However, there is significant criticism from some service members and leaders that the expedited transfer would damage a perpetrator's career as there would be a presumption of guilt before their case was adjudicated by the military justice system (Namrow et al., 2016a). 


\section{Training}

Consistent with other aspects of this organization's culture, training has been the most visible and common response of the military's MST strategy, which servicemembers begin receiving from entry into the military throughout their career. Training has also received the most criticism and recommendations for improvement by service members. Initially, sexual assault prevention training in the military was dependent on promoting risk-reducing strategies like self-monitoring levels of alcohol. However, as the DoD research and training evolved, bystander intervention has become an emerging cornerstone in prevention efforts. As with the civilian sector (Coker et al., 2015; McMahon, 2010), the significance of adopting bystander intervention strategies is that it shifts the role for speaking up and intervening from the individual to the collective group or military unit.

The chief complaint communicated through focus groups and surveys is the quality and effectiveness of the training in preventing sexual assault. Although SAPRO provides training guidelines across the Armed Forces, each branch of service has a significant amount of autonomy in its implementation due to their varying operational missions. Oftentimes, sexual assault prevention training consists of PowerPoint presentations and may or may not include a facilitated discussion afterwards. Consequently, the level of engagement is often contingent on the prioritization of the individual branch of service. Where most service members and cadets/midshipmen gain valuable information from training about reporting options and victim support services available, they often complain about the desensitization and apathy that often comes as a 
result of overtraining on a subject that does not offer new information or diversify how the subject matter is delivered or engaged.

The commonality of feedback that came from both the service members and cadets/midshipmen in the military academies is that the most effective training has included a variety of role-playing and small group discussions. Respondents also expressed interest in having guest speakers such as legal professionals, sexual assault survivors, and, if possible, perpetrators convicted of sexual assault (DMDC, 2014, 2016). Military Justice System

Service members are skeptical about the treatment from their chain of command. However, there is an even greater fear of ostracism, social retaliation, and maltreatment from their own peers as a result of reporting an incident and getting their peers in trouble, thus damaging their career. Service members are concerned about punitive administrative action related acts to the sexual assault, such as underage drinking, fraternization, and adultery (Namrow et al., 2016a; 2016b, RAND National Defense Research Institute, 2014).

Another prominent theme in the DoD literature identifies retaliation as the most significant barrier to victims reporting incidents of MST, but especially sexual assault incidents. In addition to the potential loss of privacy, the perceived and real threats of retaliation serve as barriers to reporting incidents to supervisors through the chain of command (DMDC, 2014; Namrow et al., 2016a). Professional retaliation is associated with being assigned less desirable taskings and the loss of training opportunities or job advancement. Social retaliation includes behaviors ranging from being shunned by peers 
to experiencing verbal harassment and threats of violence. Additionally, the threat of bullying, ostracism, and social retaliation is compounded by the availability of social media, which has become a modern tool used to prevent victims from reporting the incident and/or moving forward in the military justice process (Namrow et al., 2016b, RAND National Defense Research Institute, 2014).

\section{Gap Between the Department of Defense's Data and the Survivor Experience}

A powerful supplement to the DoD reports is the 2013 and 2014 survivor testimonies before Congress, which provided a human face to the pervasive incidents of sexual harassment and sexual assault across the branches. I reviewed the television testimonies and transcripts of two separate hearings conducted in 2013 and 2014 before the Senate Armed Forces Subcommittee. The hearings included four female and two male survivors of sexual assault from various branches of military service. In addition to discussing their direct experiences with sexual assault and sexual harassment in the military, the survivors gave a personal account of the adverse, and sometimes retaliatory, treatment by their chain of command and peers once they reported the incidents. Additionally, the survivors also discussed related topics such as PTSD, suicide attempts, and traumatic brain injuries that directly resulted from their sexual assault and the obstacles they faced when seeking treatment services and support from the military justice system (CSPAN, 2013, 2014; United States Congress, 2014). Lastly, the survivors provided recommendations for reducing sexual violence and increasing accountability across the military service branches 
There were three key themes that stood out in all six of the MST survivors' testimonies before Congress and which revealed several gaps between the military's stated policies about sexual violence prevention and response and the reality of the survivor's experience once they came forward to report the incident.

\section{Lack of Accountability and Retaliation}

The most prominent theme that came from all eight of the survivors was that the perpetrators of the sexual assaults were not held accountable and brought to justice for their actions. In some cases, the perpetrators were allowed to stay in the military, and were sometimes even promoted. The perception was that the military was still seen as an old boys' club where the careers of the perpetrators and leaders within the chain of command, were prioritized over that of the victims who were systematically silenced and discouraged from coming forward and filing charges.

Regardless of the demographics of the survivor in terms of gender, rank, and branch of service, there was a strong and unified message that the military justice system needs to be significantly reformed. There was support for the passage of the Military Justice Improvement Act, which would automatically remove sexual assault cases to a specially trained prosecutor outside the victim and perpetrator's chain of command to minimize conflict of interest and improve impartiality (Office of Senator Kirsten Gillibrand, 2009).

There has been a growing frustration that any gains in increased accountability for the perpetrators have not come from the military but from the survivor community, veteran advocacy groups, and legislators, despite the military's claims of zero tolerance 
and self-policing (González-Prats, 2017). The survivors also cited a lack of access to redress in the civilian courts as problematic in seeking justice and accountability from their perpetrators (CSPAN, 2013, 2014).

Social and professional retaliation were also identified as significant deterrents to reporting sexual assault and moving forward in the justice system. Survivors shared their experiences of retaliation in the forms of continued sexual harassment and ostracism by the members in their unit, being denied access to treatment, and being discharged from the military for a personality disorder, which would negatively affect their ability to access the full range of medical, educational, and other benefits to which they were entitled (CSPAN, 2013, 2014).

\section{Gender Identity and Military Culture}

Another theme that came from the congressional testimony was the absence of men as sexual assault victims in the larger discourse about MST. The two male survivors spoke of humiliation of the attack as well as the disbelief and humiliation they were subjected to once they reported the incidents to their chain of command. The male survivors spoke of the isolation they felt in trying to get counseling and treatment because of the lack of resources for male survivors (United States Congress, 2014; CSPAN, 2013, 2014).

Like their male counterparts, women survivors were subjected to the disbelief prevalent in the military's hyper-masculine culture that tolerates discrimination, harassment, and exploitation. The survivors expressed concern that a culture that sees women as weak, less-than, and as responsible for their own victimization will not change 
until perpetrators are held accountable and brought to justice (United States Congress, 2014; CSPAN, 2013, 2014).

\section{Training and Messaging}

As identified by the DoD literature, the quality and effectiveness of the military's training in preventing sexual assault was heavily criticized by the survivors. Many found the current format used by the military as a perfunctory "check the box...sign here...move on" (CSPAN 2013, 1:21:07-1:21:10) that is often not put into practice by their commanders and chain of command. They described the messages included in the training and accompanying print material like a former slogan used, "Ask her, when she is sober," as problematic and rooted in victim-blaming and rape myths versus facts and accurate data. Oftentimes, the emphasis is placed on the victimhood and sexual vulnerability of women while concurrently ignoring the reality of male victimization (United States Congress, 2014; CSPAN, 2013, 2014). Additionally, placing the emphasis of prevention training on risk education about alcohol and potential misunderstandings or lapses of judgement places the responsibility on the victim, instead of the perpetrator, where it should be. In the words of one of the survivors, "Messaging is critical. Culture change can't happen without an entire rethinking of how this is messaged in the military" CSPAN, 2013, 1:22:35-1:22:45).

The totality of the survivors' experience speaks to the need for a continuous and critical evaluation of the effectiveness of MST prevention and response efforts. It also leads to the main questions relevant to the continued progress of critically examining MST prevention and response efforts: What would the military's prevention and response 
efforts look like if the survivors of sexual harassment and sexual assault were the ones designing and evaluating the effectiveness of the training? How might be aligned with the current military training, how might it differ? These questions serve as the impetus for identifying survivors as critical strategic partners in improving the military's sexual harassment and sexual assault prevention and response efforts.

\section{Summary of the Literature}

The triangulation of the empirical data, DoD reports and MST survivor testimonies before Congress provided a foundation to approach sexual violence prevention within the military, specifically within the areas of intersectionality, gender identity, and messaging.

Although the totality of the studies and reports provided individual demographic information of the study participants, there was an absence of attention paid to the intersecting identities and power dynamics that exist with acts of sexual harassment and sexual assault.

Organization cultures like the military that value uniformity and heterogeneity, minimize and ignore the role other identities such as race, ethnicity, gender, religion, and sexual orientation play in victimization and the perpetration of sexual violence. The key finding came from Suarez and Gadalla's (2010) meta-analysis of 37 studies that found a positive correlation between RMA and other discriminatory worldviews besides sexism, such as racism, heterosexism, ageism, and religious intolerance, thus making a case for paying closer attention to intersectionality and multiculturalism in prevention programming. 
There are several key findings within the gender identity literature and the survivors' testimony that underscore a need to pay increased attention to this area within sexual violence prevention. This is especially timely and significant because of recent military policy changes allowing women and openly gay and transgender service members the unrestricted ability to serve in all occupational specialties just as their cisgender male heterosexual peers.

The survivor testimonies and literature reviewed confirms that there is a stronger propensity for sexual inequality, strict gender role adherence, and a normalization of sexual violence in cultures where men are the standard bearers of power, privilege, and status. Furthermore, when discourse surrounding sexual harassment and sexual assault continues to exclusively focus on the female victim-male perpetrator paradigm, it contributes to an acceptance of rape myths that hurts all victims.

In the area of messaging, there are three areas from the literature, DoD reports, and MST survivor testimonies which provide useful insight into the development of sexual violence prevention programs.

First, as Mejia and colleagues (2015) and the DoD (2014) acknowledge, acts of sexual harassment and sexual assault run along a more comprehensive continuum of harm or sexual violence, which runs contrary to the traditional view that such acts are independent or unrelated incidents and disconnected from other forms of interpersonal violence.

Second, the cornerstone of communication is framing, which not only influences how individuals and communities understand an issue but can contribute to how they 
conceptualize potential solutions. Traditionally, sexual violence has been exclusively framed as a criminal justice issue with a focus on punitive actions against the perpetrator instead of a public health issue that pays attention to the larger community factors that play into the continued prevalence of sexual violence.

Third, the testimonies of the MST survivors confirmed that, despite the military's explicit zero tolerance messaging against sexual harassment and sexual assault, there still exists a significant gap between what is communicated in MST prevention programs and the reality experienced by their peers, leaders, and overall military unit once they report the incident. This experience includes professional or social retaliation. This last detail underscored the need for increased survivor voice in sexual violence prevention. 


\section{Chapter Three: Methodology}

This chapter consists of four sections that identified my study design and methods, and the data collection and analysis process. Additionally, my positionality and approach as a researcher, and the ethical issues that were considered when engaging in research with trauma victims is covered.

\section{My Positionality as a Researcher/ Research Approach}

Regardless of my research subject matter, the strategy I have found most useful was identifying and grounding myself in my positionality and research approach (Ackerly \& True, 2010). This included identifying the paradigm that guided my thinking and direct action as well as best represented my worldview (Mertens, 2014). Many survivors of MST exist within the intersection of various systems of oppression, namely rank, gender, race, ethnicity, and socio-economic status.

Using Lincoln and Guba's (2000) four-quadrant framework of paradigms (positivist, postpositivist, constructivist, and critical theory) as a guide, I chose to use the critical theory paradigm. It resonated with me because, ultimately, it aims to "disrupt and challenge the status quo" and because it is based on the premise that the lived experiences of both the researcher and the participant are heavily influenced by social and historical dynamics, and institutions, of power (Ponterotto, 2005, p.129).

Using Lincoln and Guba's (2000) three defining questions of a paradigm, I was able to dig below the surface and identify the ontological, epistemological, and methodological viewpoints that guide my process of inquiry and knowledge-making. The ontology, or the nature of reality and being (Lincoln \& Guba, 2000), exists within the 
critical theory paradigm and recognizes that values such as gender, ethnicity, and culture shape reality, which is concurrently influenced by social and historical power relations (Ponteretto, 2005). The epistemology focuses on the relationship between the research participant and researcher (Ponteretto, 2005). Whereas objectivity is achieved in the positivist and post-positivist epistemologies by the researcher striving for neutrality and avoiding personal values or biases, the relationship between the researcher and the participant(s) is intentionally interactive, and it "should be empowering to those without power" (Mertens, 2014, p. 32).

Finally, the axiological or ethical considerations in this paradigm prioritize collaboration, inclusion, and social justice via equal opportunity (Mertens, 2014; Morris, 2006). As the subject of MST is personal to me, I feel the challenge of honoring and doing justice to the research participants, the observed, and myself. This "crisis of representation" (Hole, 2007, p. 697) presents the conflict that faces many social research scientists: how do they simultaneously honor their own truths and positionality, while engaging others with different views from their own?” (Hole, 2007; Takacs, 2003).

\section{My Positionality}

My positionality includes intersecting identities (Carbado, Crenshaw, Mays, \& Tomlinson, 2013; Kezar, 2002) that influence my worldview and have an impact on how I interact with others, especially negotiating the "space in between" (Corbin Dwyer \& Buckle, 2009, p. 54) being an insider and outsider researcher (Acker, 2000; Bourke, 2014; Kezar, 2002). 
I identify as a Latina, heterosexual, cisgender female, and an Army veteran. Reflecting on my position and privilege in my professional life to date, I acknowledge that the marginalization I have experienced has been primarily attributed to being a woman in the workforce, especially serving in male-dominated occupations like the military, which is germane to the topic of this dissertation and central to my motivation for investigating MST (Acker, 2000; Bourke, 2014).

My service in the Army, both in the Army Reserves as an enlisted soldier and, later, as an officer on active duty is rife with conflicting emotions as I reflect on the 5year tenure of my service from 1998 to 2004. On one hand, serving in the military was one of the most empowering experiences I have had in my life. The personal and professional challenges that tested my will and resilience combined with the unique bonds that I forged with other service members made my joining the Army after college at 23 years of age one of the best decisions I have made in my life.

On the other hand, my own experiences with gender discrimination and sexual harassment while advocating on behalf of sexual assault victims was disheartening and, more often than not, infuriating. When I realized that the military's culture of camaraderie, integrity, and accountability, which espouses such values as good order and discipline, zero tolerance, and leaving no man behind, applied only to a few within the military, it significantly affected my decision to prematurely end a promising Army career after my initial contract.

My identity as an Army veteran continues to reconcile the nuanced emotions of love, pride, and belief in the military as an institution with the disappointment and disgust 
I feel with the military's weak and superficial response to the continued prevalence of discrimination, sexual harassment, and sexual assault, which feels like a tacit acceptance of defeat and a normalization of victims as collateral damage.

As both a veteran and a researcher, I approached this investigation with four beliefs. The first is that the military's MST prevention and response efforts have been more focused on service members avoiding victimization and for survivors to survive better than on identifying and bringing perpetrators to justice. The second is that survivors of MST continue to be an afterthought in the prevention and response strategy

of incidents of sexual harassment and sexual assault. The third is that the military has the skill, talent, and capacity to reverse the trajectory of MST. The fourth is that my intersecting identities as a military veteran and researcher concurrently place me both in the insider and outsider categories in this study.

\section{Inside/Outside Researcher}

The subject of MST is a personal one for me. As a junior officer who directly experienced sexual harassment and the intimidating process of reporting the offending commander to my chain of command, a friend and colleague of his, I am well aware of the stigma attached to defending yourself and informing on a fellow service member. Additionally, as someone who frequently occupied positions of leadership and responsibility, I often served as an advocate for soldiers in our unit who were sexually harassed and/or sexually assaulted, raising awareness to other commanders and challenging common responses rooted in antiquated rape myths such as alcohol, culture 
of promiscuity, and lapse of individual judgment (Childress, 2013; DoD, 2014; Mejia et al., 2015; RAND National Defense Research Institute, 2014; 113th Congress, 2013)

As a result, I consider myself as having an insider status in this study for four reasons. The first is that I am a military veteran who has served both as an enlisted member in the reserves and as an officer on active duty. The second is that I am still actively involved in the military/veteran communities as a mentor and advocate in transitioning issues. The third is that I have experienced gender discrimination and sexual harassment in the military, and the fourth is that I understand the military justice system and how cases of sexual harassment and sexual assault are handled.

Despite my insider status, I recognize that I can also be considered an outsider. This is due to the fact that I left the Army in 2004, and I am no longer serving in the reserves or National Guard. Also, I have not directly experienced sexual assault and the personal or professional stigma attached to it in the military. My identity as a cisgender female and Latina with features that allow me to pass as White provide me with the privilege and protection from the compounding marginalization that occurs for more visible members of color and/or those who identify as LGBTQ. Lastly, I am a Ph.D. student conducting research on a very personal and painful topic.

Instead of being caught up in the advantages and disadvantages of being an insider or outsider researcher and attempting to create an artificial boundary of objectivity (Corbin Dwyer \& Buckle, 2009; Kanuha, 2000), I am committed to recognizing the power of personal connection and knowledge-making that is available in straddling both worlds (Acker, 2000; Corbin Dwyer \& Buckle, 2009; Kanuha, 2000). "The insider- 
outsider question cannot be fully resolved and that perhaps it is necessary not to bring this issue to a close but to find a way to work creatively, but within the tensions created by the debate" (Acker, 2000, p. 189).

Drawing from the insider/outsider research literature, I understand that the benefits I draw from being an insider, such as immediate and facilitated access along with rapport and understanding, will also present with unique challenges that outsider researchers may not face (Acker, 2000; Corbin Dwyer \& Buckle, 2009; Kanuha, 2000). Such challenges include over-identifying with the participants' experiences, projecting similar experiences onto the participant, and a propensity for increased assumptionmaking because of inside information, common language, and culture (Acker, 2000; Corbin Dwyer \& Buckle, 2009; Kanuha, 2000).

Social science and qualitative researchers have provided strategies for these challenges and for learning how to occupy "the space between" (Corbin Dwyer \& Buckle, 2009, p. 61). First, it is strongly recommended that researchers be engaged in continuous reflexivity and disciplined bracketing, disclosing viewpoints and biases so as to be able to increase the transparency and accountability of the impact of the researchers' positionality on the collective investigative process (Acker, 2000; Bourke, 2014; Corbin Dwyer \& Buckle, 2009; Kanuha, 2000).

Second, Kahuna (2000) noted that a key area of reflexivity is being aware of making assumptions as an insider researcher during an interview because of common language, culture, and customs. One way to check these assumptions is to ask follow-up questions and further clarification when the study participant implies or explicitly states 
that the researcher must know what they mean. Additionally, a helpful tip provided is to probe the participant for exceptions to absolutist statements (Kahuna, 2000), which, in this type of study, might sound like "you know how all officers or commanders can be." This provides further clarification about an issue, so it is on the record rather than assumed.

Third, although not explicitly framed as a strategy to mitigate insider subjectivities, Acker (2000) identified the added value of an insider researcher working on a team with other researchers who either are outsiders or are also negotiating "the space in between" (Corbin Dwyer \& Buckle, 2009, p. 62) and who can provide peer feedback and debriefing for ongoing uncertainties and dilemmas facing researchers (Corbin Dwyer \& Buckle, 2009).

\section{Ethical Considerations as a Researcher}

It is important to note that individual trauma can occur as an isolated or recurring event, and the level of trauma is dependent on how the individual experiences the trauma. There are numerous forms of trauma such as war, child abuse, accidents and environmental disasters (Gitterman \& Heller, 2011; Sayer et al., 2009). All forms of trauma share common benefits and risks in related research (Becker-Blease, 2006; Cromer \& Newman, 2011).

I used the intersectional framework as a lens (Carbado, 2013; Cho et al., 2013; Hancock, 2007) incorporating the data from the literature review as well as my personal experiences as an Army veteran and personal experiences with MST to provide additional insight. Engaging survivors of MST required an intentional and thoughtful intersectional 
approach to minimize the institutionalized betrayal and maximize the strength-based tenets of autonomy and self-determination.

\section{Weighing the Risk and Benefits of Trauma-Related Research}

The common thread that runs through numerous studies in trauma-related research is that there are as many benefits to be gained as there are risks to the participants involved (Cromer, 2011). When contemplating the risks and benefits of this type of study, it is advantageous for the researchers to adopt multiple research methodologies to proactively identify ethical concerns relevant to their participants and all involved stakeholders.

"There is increasing value being placed on the ethical value of respecting individuals' rights to autonomy and self-determination...this should be weighed against first doing no harm" (Behnke, 2004, as cited in Cromer, 2011, p. 1543). A researcher needs to understand the risks of re-traumatization, fragilization, and dual relationships (Ackerly \& True, 2010; Cromer, 2011; Newman, Rish, \& Kassam-Adams, 2006), with the potential benefits of validation, inclusivity, and the accessibility of resources (BeckerBlease \& Freyd, 2006: Newman, Risch, \& Kassam-Adams, 2006).

Occupying intersecting roles as both a social worker and a researcher, I acknowledge that I needed to serve as both a healing presence and compassionate witness throughout the recruitment and data collection process while understanding that I am not occupying a therapeutic role.

The primary obligation of the therapist is to promote the welfare of the client, the priority of the researcher is to meet the aims of the research study...how do we 
ensure that a client is able to assert him/herself within the therapy situation with regard to the dual role of the client research participant. (Hart \& CrawfordWright, 1999, p. 208)

The dual roles of researchers and direct service providers, such as therapists and social workers, present ethical dilemmas because of the ambiguity regarding expectation of privacy, informed consent, and the existing dynamic of power. These blurred lines lend themselves to potential exploitation and a complicated sense of accountability, which may compromise the researcher and the participant. This ethical dilemma was considered during the research design phase, especially when discussing the venues and methods of recruiting research participants (Ackerly, 2010). I achieved this delicate balance of roles by clearly identifying boundaries for myself as a researcher. In addition, I ensured that I had the resources and support ready for the study participants throughout the study, from the screening stage, through the informed consent stage, as well as throughout the interview process.

Additionally, an important consideration that is not always explicitly included in research protocols or review board safeguards is the proper self-care for researchers and their team, who may experience secondary trauma or "compassion fatigue" (Lipsky \& Burke, 2009, p.3.) "via their role in bearing witness to participants' accounts of tragedy." (Newman et al., 2006, p. 40). This is especially a concern for researchers who share similar backgrounds with survivors and/or have experienced trauma themselves 


\section{Implication for Research and Further Studies}

Using intersectional research principles and increased self-reflexivity, researchers have the opportunity to expand their knowledge base regarding MST, so they can offer empowerment and healing through the research participation process. Ackerly (2010) stated that "researchers must be able to design research projects in ethical ways that attend to hierarchy without exploiting hierarchies... our research and our presence as researchers can change relationships among community members, potentially challenging or reinforcing hierarchies" (p. 13).

The need exists for researchers and their surrounding communities to be as selfreflexive as they are ethical and inclusive, as the concurrent issues of MST, women in combat roles, and the continued controversy regarding the service of transgender members continue to be in the national spotlight (CSPAN, 2017; De Vogue, 2017; González-Prats, 2017; Halloran, 2013; Montgomery, 2013; Peralta, 2013; Seck, 2015; Wire Reporters, 2015). "Ethical reflection is about responsibility and humility. Ethical reflection in research is about the researcher developing and maintaining an ethical compass that informs and guides her decisions in anticipated and unanticipated dilemmas of research" (Ackerly \& True, 2010, p. 11).

\section{Study Design and Methods}

Upon approval by my dissertation committee and the Portland State University Institutional Review Board, I conducted a qualitative study of 21 MST survivors, using a nationwide sample. I conducted participant outreach via my existing network of personal and professional contacts, to include various branches of the military, the Portland VA 
Medical Center, Homeless Veteran Program, and the Oregon Department of Veteran Affairs. Additionally, I solicited participants through closed Facebook pages, such as Latinas Completing Doctoral Degrees, Progressive Veterans and Military Families, Veteran 2 Veteran, Minority Veterans of America, and Wise Latinas Linked.

As the experience of MST survivors plays a central role in this study, the qualitative tradition that best aligned with the goals and objectives of the dissertation is phenomenology, which aims to "understand the hidden meanings and the essence of an experience together with how participants make sense of these" (Gribich, 2013, p. 92). Two significant principles of phenomenology are that (a) there is not just one reality of how particular events or phenomena are experienced by individuals (Hesse-Biber \& Leavy, 2006), and (b) the interaction between the researcher and the respondent (as with between researchers and text) contributes to knowledge-generation and meaning-making of the phenomena (Gribich, 2013). What makes phenomenology such a dynamic approach to explore lived experiences of individuals is that it is versatile enough to be used in concert with other analytical approaches (Gribich, 2013), and/or the practice of bracketing, where investigators explicit identify and avoid any viewpoints, biases, and prejudices which may have an impact on the research (Hesse-Bieber \& Levy, 2006). In phenomenology,

the role as a researcher is to provide transparency of process and to bring the reader as close as possible to the experiences and structures of the essences being displayed and this will depend on creativity of re-presentation and display of information gained. (Gribich, 2013, p. 100) 


\section{Research Questions}

This dissertation study is grounded in the following three research questions,

1. What was the messaging (via the military's prevention and response efforts) that survivors received about MST from their chain of command and their military unit overall? How did survivors internalize this messaging and how did it impact their experience of MST?

2. What roles (if any) do gender identity and attitudes towards gender play into the military's messaging (e.g. prevention and response efforts) about MST?

3. What (messaging) do survivors feel would have helped? Moving forward, what would survivor-centered prevention and response efforts to MST look like?

\section{Population and Sample}

Using a pre-interview participant screening tool (Appendix A), I identified my population sample. Participants met the following criteria:

- Identified as a veteran or active duty service member (Army, Navy, Marine Corps, Air Force, and Coast Guard)

- Experienced sexual harassment and/or sexual assault during military service within the past 15 years.

I purposively sampled participants using maximum variation sampling (Creswell \& Clark, 2011). This type of sampling strategy increases the depth of understanding by selecting a diverse group of individuals who are expected to have different perspectives (Creswell \& Clark, 2011). I sought to include participants who identify all along the gender identity and sexual preference continuum: women, men, non-binary, transgender, 
gender non-conforming, as so forth. I also ensured that there was variation in race, ethnicity, type and branch of military service, rank, and experience of sexual harassment and/or sexual assault. I achieved this by oversampling men, women of color, and MST survivors who identify as LGBTQ, acknowledging that some of the service members' identities might intersects two or more of these communities. My goals were to identify the following sample ranges for the participant population that identify as survivors of sexual harassment (6 to 10 participants), survivors of sexual assault (10 to 15 participants), men (10 to 12 participants), people of color (15 to 19 participants), and LGBTQ (6-10 participants).

\section{The Process of Data Collection and Analysis}

This section outlines my data collection process, as well as details my data analysis strategy. Additionally, I discuss the process I will be using to test the trustworthiness and validity of my research findings upon completion of my study.

\section{Data Collection}

I conducted semi-structured individual interviews over the telephone. All study participants received a complete study description and were given informed written consent (Appendix B) to have their interview audio-recorded for transcription. Their study description also included a list of resources available to them as veterans in anticipation that the subject matter could trigger unpleasant memories and/or emotions during the course of the interview.

At the onset of the interview protocol (Appendix C), I began with basic, icebreaker types of questions about joining the military such as asking their age, 
motivation for doing so, military occupational specialty, and the duty stations where they served in order to help ease them into talking about their military experiences and identify commonalities in service branches and/or duty stations.

Next, I transitioned to more participant-driven questions, asking them to define sexual harassment and sexual assault, to include asking them to provide multiple examples of each to provide context on how they view MST. I also asked them about the MST prevention and response training they received as well as their direct experience with sexual harassment and sexual assault. Depending on the participant's narrative, I used a variety of probes to elicit rich data regarding the messaging they received about MST from their chain of command and military unit and how the messaging affected how they experienced MST.

Lastly, I transitioned towards investigator-driven questions, soliciting their input and recommendations about what type of messaging in MST prevention training would have been helpful.

\section{Data Analysis}

My data analysis process was conducted in five stages, as shown below in Figure 2. 


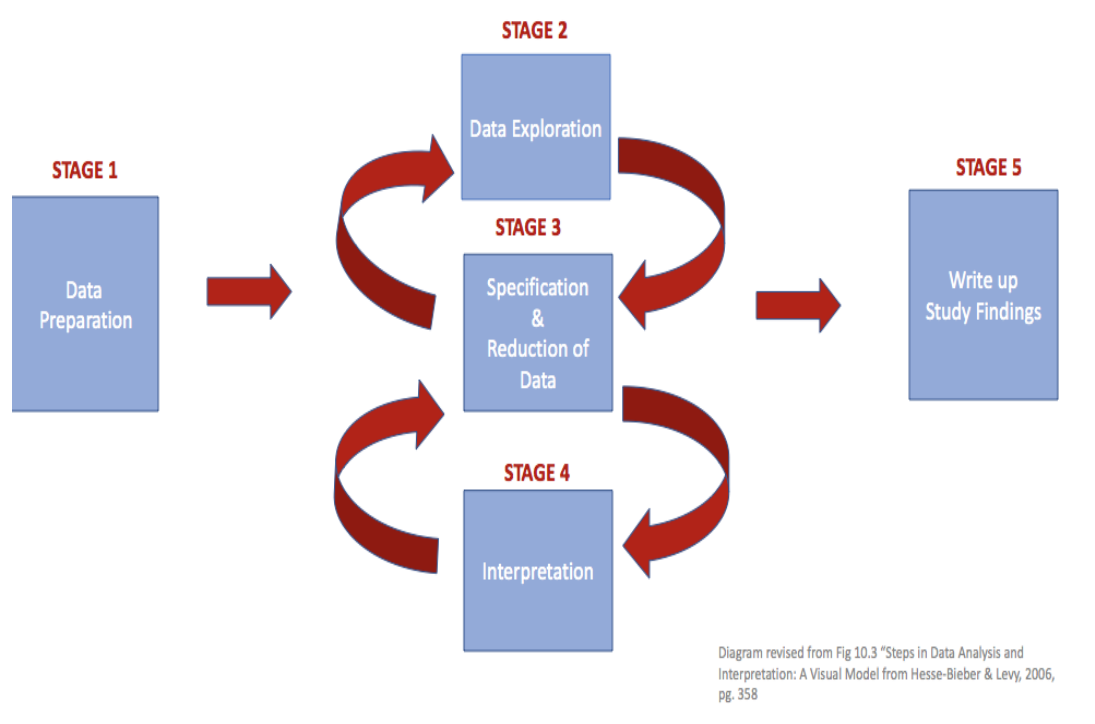

Figure 2. Data analysis process.

Stage 1: Data Preparation. As soon as the interview was completed, the raw audio file was uploaded to an electronic folder at Portland State University's Regional Research Institute (RRI), where the identifiable data for this study was kept along with the participant key, which included the participant's name, demographic data, and other relevant information from the screening tool. As screening tools were completed and the interviews were conducted, this participant key was continuously updated and permanently resides in the folder, to which only the dissertation chair and I were able to access.

The raw interview files were then transcribed by an outside transcriptionist, who provided a verbatim transcription and removed all identifying data from the final interview transcription. One original copy of the de-identified transcript was uploaded to the RRI's electronic folder, where the de-identified data from the study was kept and 
made accessible to the dissertation chair, secondary coder, the dissertation committee members, and me.

Another copy of the de-identified transcript was uploaded into the NVivo Qualitative Software program (NVivo, 2018.) for use in the data exploration stage. Additionally, my field notes were integrated into the participant profile, so as to centralize all the data.

In addition to using an outside transcriptionist, I also used Acker's (2000) recommendation for insider/outsider researchers, and identified and trained a non-veteran college student to serve as a second, and outsider, coder (Appendix E).

Stage 2: Data Exploration. Working from the NVivo database, I used the software to read the de-identified transcripts and digest the information. As I read through the transcript, I highlighted key quotes and passages, marking up the text with notes in the margin, which NVivo refers to as annotations. While I read through the transcripts, I considered "what is going on here, what fits together, what does not fit?"

Looking at the totality of the individual transcript with highlighted text and field notes, I created an interview summary which included the interview as well as captured my reflective impressions during and after the interview.

Additionally, every time I accessed and completed any tasks within the NVivo database, I added a date and time stamped journal entry that included a brief summary of what actions were completed, which was stored in a folder labeled "Journals".

While I read and annotated the interview text, the secondary coder simultaneously and independently conducted the same data exploration tasks on the specified interviews, 
and conferred with me about specific interviews at certain intervals of completed interviews.

Stage 3: Specification/Reduction of Data. As highlighted in Figure 2, stages 2, 3, and 4 of the data analysis was an ongoing and fluid process, which, moved organically from one phase to another.

Consequently, the secondary coder and I identified codes throughout the body of the interview text, which were "chunks or segments in the text that point to key themes, patterns, ideas and concepts that may exist within the data" (Hesse-Biber \& Levy, 2006, p. 353). Similar to the open coding approach (Charmaz, 2004) used in the qualitative data analysis tradition found in grounded theory (Glaser \& Strauss, 1967), I identified preliminary or descriptive codes (Hesse-Biber \& Levy, 2006) as I went through each line and paragraph of the text, instead of waiting until all the interviews were completed.

As we identified initial codes in each transcript, the secondary coder and I compiled, updated, and identified a list of about 20 open preliminary codes. Whenever possible, we looked for opportunities to reconcile similar codes as well as create main codes (parent codes), and corresponding sub codes (child codes; NVivo, 2018.).

As each interview transcript was read and coded, we returned to the master list of codes and refined them, which became more interpretative in nature and moved towards a more focused coding approach. Doing so "require(d) that the researcher examine(d) all the data in a category, compare(d) each piece of data with every other piece, and finally buil $(\mathrm{t})$ a clear working definition of each concept, which is then named" (Hesse-Biber \& Levy, 2006, p. 352). Additionally, as codes become more analytical, patterns of data, 
more commonly referred to as themes, emerged (Braun \& Clarke, 2006). The NVivo data management system refers to the themes as categories (NVivo, 2018).

I conducted stages 2,3 , and 4 as a parallel process with the secondary coder, working on the same transcripts simultaneously and independently. I formally conferred with the secondary coder at the 3, 5, and 10 interview mark and as needed, identifying and comparing codes and categories. I also consulted with the dissertation chair, and the other members of the committee as needed, with emerging themes and other parts of the data analysis process.

Next, we conducted a thematic analysis, "identifying, analyzing and reporting patterns (themes) within data" (Braun \& Clarke, 2006, p. 79). We used an inductive "bottom up approach" (Braun \& Clarke, 2006, p. 79) to analyze the data, looking for themes that stemmed from the data themselves, versus fitting a specific theory or code from the literature to the data (Braun \& Clarke, 2006).

In addition to identifying themes that fit a specific pattern, I (and the secondary coder) looked for anomalies that contradict some of the emerging patterns. Referred to as "negative cases," these outliers were the key to establishing a counterpoint to any patterns that may emerge and will help increase trustworthiness and craftsmanship validity, or the extent to which the researcher and their credibility are regarded by the scientific community (Hesse-Biber \& Levy, 2006).

The primary analysis was focused on identifying themes at a semantic level, searching for themes and meanings explicitly found on the surface of the interview data (Gribich, 2013). However, we noted potential latent themes, which "are theorized as 
shaping or informing the semantic content of the data" (Braun \& Clarke, 2006, p.84), and identified them as areas for follow-up studies in the future.

All throughout the theme identification process, I directly consulted with the dissertation chair and committee members to incorporate feedback and finalize themes that were expressed commonly, carried relevance to the participants, and had the potential to inform policy or programs (Milat, King, Bauman, \& Redman, 2012; Ward, House, \& Hamer, 2009).

Parallel to the coding refinement process was the process of preparing an analytical memo which consisted of written ideas about the analysis and interpretation of the data (Hesse-Biber \& Levy, 2006). Similar to the initial data exploration phase, a key part of preparing the memo was reflecting what was going on in the interview, how the codes and categories are related or unrelated, and what it all meant. "Memos are the pathways into your data; they are an intermediate step between the coding and your interpretation and the write up of the data" (Hesse-Biber \& Levy, 2006, p. 355).

Stage 4: Interpretation. The secondary coder and I analyzed the first ten interviews, honing specifically on the established themes/categories, the negative cases that contradicted the themes or theories, and all of the memos and interview summaries created in the analysis process.

Throughout the interpretation process, I utilized the following areas of the Kvale (1996) validity checklist (Hesse-Biber \& Levy, 2006, p. 359) to help me determine if I was addressing trustworthiness or am ready to move from the interpretative phase to the writing findings stage: 
- Are you telling a convincing story?

- Try theorizing from your data interpretations

- Have you reached your findings with integrity-have you checked your procedures?

- Look for negative cases

- Make your interpretations available for discussion (agreement and debate) among "legitimate knowers" (others in the social scientific community).

- How do your findings impact those who participated in the research, and how do your findings impact the wider social context in which the research occurred? Once the data analyses are completed and the preliminary interpretation, I conferred with the dissertation chair about the findings to determine if I was ready to move forward to write up the findings.

Stage 5: Write up Findings. In addition to writing up my findings for this dissertation, I am also looking forward to preparing a manuscript for a peer-reviewed scientific journal to share the findings. Doing so will help contribute to the communicative validity, where "each interpretation of a given finding is open to discussion and refutation by wider community of researchers and the relevant community" (Hesse-Biber \& Levy, 2006, p. 64).

\section{Proposed Next Steps in Increasing the Trustworthiness and the Validity of This}

\section{Research}

The last part of the triad of Kvale's (1996) criteria of validation is pragmatic validity, which is to consider the research findings within a context larger than that in 
which the study was conducted, especially being aware of where the power dynamics for policy change lies for MST-related research (Hesse-Biber \& Levy, 2006). As an early investigator, I see the best opportunity to enhance the validity and trustworthiness of the research study and the findings, and impact social change in MST-related policies is to use this dissertation as the first step in the triangulation process of understanding the lived experiences of MST survivors (Hesse-Biber \& Levy, 2006).

As part of my fellowship with OHSU Oregon Clinical Translational Research Institute, I will use these findings to apply for an early investigator career award with the National Institutes of Health. I foresee the next step of the triangulation as using the findings from this study to follow up using focus groups as a form of member checking to see what areas resonated with the participants and, even more importantly, which findings contradict their lived experiences. Ultimately, I would like to conclude the triangulation of data by using the data from the interviews and the focus groups to conduct a content or discourse analysis to gain a deeper understanding of the narratives in the military about MST and how survivors and perpetrators internalize various, often contradictory, messages from their military chain of command. 


\section{Chapter Four: Findings}

I conducted 21 phone interviews with MST survivors representing a variety of racial/ethnic demographics, military branches, service periods and type of MST experienced (see Table 1 below).

Table 1

\section{Participants' Demographics}

\begin{tabular}{|l|c|c|}
\hline \multicolumn{1}{|c|}{ DEMOGRAPHIC } & $\mathbf{n =}$ & $\mathbf{\%}$ \\
\hline Gender Identity & 20 & $95 \%$ \\
\hline Women & 1 & $5 \%$ \\
\hline Men & 1 & $5 \%$ \\
\hline Transgender & $\mathbf{2 2 *}$ & $>\mathbf{1 0 0 \%}$ \\
\hline Total & & \\
\hline Sexual Orientation & 17 & $81 \%$ \\
\hline Heterosexual/Straight & 3 & $14 \%$ \\
\hline $\begin{array}{l}\text { Lesbian Gay Bisexual } \\
\text { Queer (LGBQ)*31 }\end{array}$ & 1 & $\mathbf{1 0 0 \%}$ \\
\hline No response & $\mathbf{2 1}$ & $81 \%$ \\
\hline Total & 16 & $19 \%$ \\
\hline Race and Ethnicity & & $5 \%$ \\
\hline $\begin{array}{l}\text { White and Non- } \\
\text { Hispanic Latino(a) }\end{array}$ & 1 & $10 \%$ \\
\hline Hispanic/Latino(a) & & $>\mathbf{1 0 0 \%} * \mathbf{3 3}$ \\
\hline $\begin{array}{l}\text { Native } \\
\text { American/Alaskan } \\
\text { Native }\end{array}$ & 2 & \\
\hline Multiracial & & \\
\hline Total & & \\
\hline & & \\
\hline
\end{tabular}

30 Indicates Totals $>$ the sample of 21 and/or $100 \%$ indicates that the survivor identified in several ways (i.e. Transgender AND Male)

31 Includes survivors who identified as a lesbian, gay, bisexual, or queer

32 Some multiracial candidates checked more than one category (i.e. Native American, Multiracial, and/or White)

$33 *$ Indicates Totals $>$ the sample of 21 and/or $100 \%$ indicates that the survivor identified in several ways (i.e. White, and Hispanic) 


\begin{tabular}{|c|c|c|}
\hline \multicolumn{3}{|l|}{ Educational Status } \\
\hline Some College & 3 & $14 \%$ \\
\hline Associates Degree & 5 & $24 \%$ \\
\hline $\begin{array}{l}\text { Bachelors/4-year } \\
\text { Degree }\end{array}$ & 4 & $19 \%$ \\
\hline Masters Degree & 9 & $43 \%$ \\
\hline Total & 21 & $100 \%$ \\
\hline \multicolumn{3}{|l|}{ Military Status } \\
\hline Active Duty & 5 & $24 \%$ \\
\hline Veteran & 16 & $76 \%$ \\
\hline Total & 21 & $100 \%$ \\
\hline \multicolumn{3}{|l|}{ Branch of Service } \\
\hline Army & 14 & $66.6 \%$ \\
\hline Navy & 1 & $5 \%$ \\
\hline Air Force & 6 & $28.5 \%$ \\
\hline Total & 21 & $100 \%$ \\
\hline \multicolumn{3}{|l|}{$\begin{array}{l}\text { Types of MST } \\
\text { experienced }\end{array}$} \\
\hline Sexual Harassment & 1 & $5 \%$ \\
\hline Sexual Assault & 5 & $24 \%$ \\
\hline $\begin{array}{l}\text { Sexual Harassment and } \\
\text { Sexual Assault }\end{array}$ & 15 & $71 \%$ \\
\hline Total & 21 & $100 \%$ \\
\hline \multicolumn{3}{|l|}{ Age Range (yrs.) } \\
\hline $20-30$ & 2 & $10 \%$ \\
\hline $31-40$ & 13 & $62 \%$ \\
\hline $41-50$ & 4 & $19 \%$ \\
\hline $51-60$ & 1 & $5 \%$ \\
\hline No Response & 1 & $5 \%$ \\
\hline Total & 21 & $100 \%$ \\
\hline \multicolumn{3}{|l|}{$\begin{array}{l}\text { Rank at time of MST } \\
\text { incident(s)* }\end{array}$} \\
\hline $\begin{array}{l}\text { E-1 to E-4 } \\
\text { (Lower enlisted) }\end{array}$ & 16 & $76 \%$ \\
\hline Cadet (ROTC) & 1 & $5 \%$ \\
\hline $\begin{array}{l}\text { E-5 to E-9 } \\
\text { (Non-Commissioned } \\
\text { Officer/NCO) }\end{array}$ & 13 & $57 \%$ \\
\hline $\begin{array}{l}\text { O-1-O-3 } \\
\text { (Junior Commissioned } \\
\text { Officer) }\end{array}$ & 3 & $14 \%$ \\
\hline $\begin{array}{l}\text { O-4 to O6 } \\
\text { (Field Grade Officer) }\end{array}$ & 1 & $5 \%$ \\
\hline
\end{tabular}




\begin{tabular}{|c|c|c|}
\hline $\begin{array}{l}\text { WO1-CW2 } \\
\text { (Warrant Officer) }\end{array}$ & 1 & $5 \%$ \\
\hline Total & $35 * 34$ & $>100 \% *$ \\
\hline \multicolumn{3}{|l|}{$\begin{array}{l}\text { Total Years of Active } \\
\text { Duty Service }\end{array}$} \\
\hline $\begin{array}{l}3 \text { years or less of } \\
\text { service }\end{array}$ & 5 & $24 \%$ \\
\hline $4-8$ years of service & 7 & $33 \%$ \\
\hline $9-16$ years of service & 4 & $19 \%$ \\
\hline $17-25$ years of service & 4 & $19 \%$ \\
\hline $\begin{array}{l}\text { Over } 25 \text { years of } \\
\text { service }\end{array}$ & 1 & $5 \%$ \\
\hline Total & 21 & $100 \%$ \\
\hline \multicolumn{3}{|l|}{$\begin{array}{l}\text { Geographical } \\
\text { Representation }\end{array}$} \\
\hline $\begin{array}{l}\text { Northeast } \\
\text { (PA, VA, MD) }\end{array}$ & 4 & $19 \%$ \\
\hline $\begin{array}{l}\text { Southeast } \\
(\mathrm{KY}, \mathrm{NC}, \mathrm{FL})\end{array}$ & 5 & $24 \%$ \\
\hline Midwest $(\mathrm{CO}, \mathrm{MO})$ & 2 & $10 \%$ \\
\hline Southwest (AZ, TX) & 3 & $14 \%$ \\
\hline West Coast (OR, CA) & 6 & $29 \%$ \\
\hline No Response & 1 & $5 \%$ \\
\hline Total & 21 & $100 \%$ \\
\hline
\end{tabular}

The following model represents the four themes and corresponding sub-themes that emerged from the primary source survivor interviews. In each of the following sections, the survivors shared their experiences with sexual harassment and/or sexual assault while serving, how they were treated by their chain of command and/or military unit, deficiencies in the military's prevention and response efforts, and described what a supportive environment looks like. The chapter concludes with explicit survivor-provided

34 Indicates Totals $>$ the sample of 21 and/or 100\% indicates that the survivor experienced more than one type of MST incident throughout their career, and at varying ranks and points in their careers. 
recommendations for how prevention and response services can be improved as well as how future policies can be shaped to better support survivors.

Although the model reflects four separate findings, there was a significant amount of overlap among the sub-themes. As a result, their interconnectivity and interdependence are reflected in the survivors' description of what a supportive environment looks like, and recommendations for improvement.

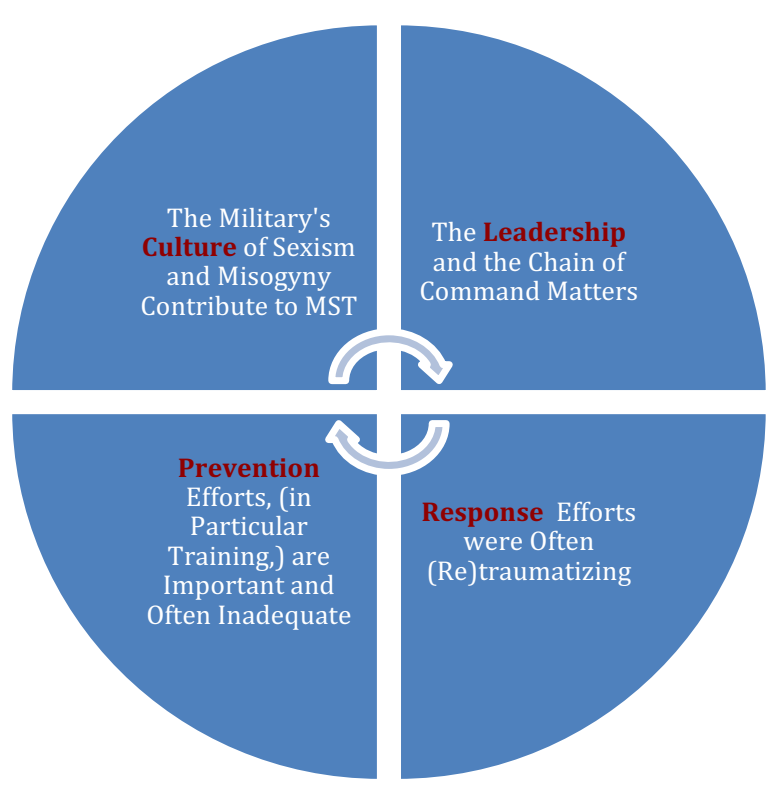

Figure 3. Themes 
Table 2

Themes and Subthemes

\begin{tabular}{|c|c|c|}
\hline Theme \# & Description of Theme & Sub-Theme \\
\hline 1 & $\begin{array}{l}\text { The military's culture of } \\
\text { sexism and misogyny } \\
\text { contribute to MST }\end{array}$ & $\begin{array}{l}\text { - The Impact of Identity } \\
\text { - Survivors' Experiences with MST are } \\
\text { minimized/ignored within Romantic } \\
\text { Relationships } \\
\text { - The "Normalization" of MST incidents } \\
\text { - Victims are often Bullied and Slut-Shamed }\end{array}$ \\
\hline 2 & $\begin{array}{l}\text { The leadership and the } \\
\text { chain of command matters }\end{array}$ & $\begin{array}{l}\text { - Leaders are Responsible for the Command } \\
\text { Climate of the Military Unit } \\
\text { - Inconsistent Treatment for Women vs. Men by } \\
\text { the Leadership } \\
\text { - Survivors Experience Professional Retaliation } \\
\text { for Rejecting Advances and/or Reporting } \\
\text { Incidents } \\
\text { - The Career of the Perpetrator is often } \\
\text { Prioritized over that of the victims. } \\
\text { - What a Supportive Environment Looks like to } \\
\text { Survivor }\end{array}$ \\
\hline 3 & $\begin{array}{l}\text { The military's response is } \\
\text { often (re)traumatizing }\end{array}$ & $\begin{array}{l}\text { - MST has a lifelong impact on the survivors } \\
\text { - Survivors were often treated poorly by the } \\
\text { Military and VA Healthcare Providers } \\
\text { - Discriminatory Policies like "Don't Ask Don't } \\
\text { Tell" can deter reporting incidents of MST }\end{array}$ \\
\hline 4 & $\begin{array}{c}\text { Prevention efforts are } \\
\text { inadequate }\end{array}$ & $\begin{array}{l}\text { - The Perceived Quality of the Training Content } \\
\text { - The Delivery of the Information Matters (ex. } \\
\text { timing, methods, etc.) } \\
\text { - The Skills of the Facilitators are Key }\end{array}$ \\
\hline
\end{tabular}




\section{Theme One: The Military's Culture of Sexism and Misogyny Contribute to MST}

How women are portrayed and talked about in the military makes a difference in whether they will be regarded with equality and respect or with inequality and disdain, especially from their male counterparts. The survivors identified four areas within the military that contributed to a culture of sexism and misogyny where incidents of sexual harassment and sexual assault are normalized and tolerated.

\section{The Impact of Identity}

First, survivors often felt that their identities as women, wives and mothers overshadowed their identities as equally valued members of the military. Consequently, this affected how they were viewed and treated by their leaders and peers. One participant stated,

Being a mom and a mechanic, those two things are not interchangeable where I was. I was so pregnant, and being in my hangar for work was a harm to my child. They wanted to have me like in the tool room around leaking hydraulic fluid and dumb stuff. I had to fight and pull all kinds of strings to get away from there. It was, it was that type of work harassment, not the, you know, being touched and inappropriate comments and it was, “We don't like you because you're a girl, and we're going to make your life absolutely unbearable." (36-year-old woman, Army veteran: 4 years of service)

The survivors also spoke about the adverse impact that the double standards for childcare and parenting responsibilities had on their careers, a burden imposed on women much more so than on their male counterparts. One interviewee discussed her experience: 
So, I know I was perceived as a slacker because I had to go pick up my daughter from day care. The husband never picked up the daughter from daycare. You know what I mean? Just those kinds of things where it exists a lot in normal, dual military like, and that's part of the sacrifice people make and I get that. But it's when it's with an abusive spouse and there isn't a shared responsibility of whatever, inevitably my career suffered. (44-year-old woman, Army veteran: 4 years of service)

Additionally, survivors felt that their identities as servicemembers tend to be diminished or erased because their gender is usually brought up within the context of lowered standards for physical fitness and technical competence, and/or the inability to perform job duties as the same level of their male peers. As one survivor stated, When I went through it was November ' 98 . The flights were completely malefemale integrated, and their whole thought was that, if we try to make it equal, then it'll take away this "woman can't do these things." And, to a certain extent, they were right because it did become a competition a lot of times, and any women in the military, they'll always tell you that you always have to work so much harder than any man doing the bare minimum just to even be acknowledged. I mean, there were guys that skated by doing the bare minimum and nobody gave it a second look but the second that a chick slows down for half a second, you get swarmed. And so you learn real quick, I have to be better. I have to be better than these guys if I'm going to make it here. (38-year-old woman, Air Force veteran: 7 years of service) 


\section{Survivors' Experiences with MST are Minimized/Ignored Within Romantic}

\section{Relationships}

Survivors shared that, within their dating and marriage relationships, their sexual assault experiences were either minimized or ignored entirely, effectively ignoring their ability to assert autonomy over their bodies and give consent because of a past or present sexual relationship with the perpetrator:

Even though I said no, it didn't matter because I had had a sexual relationship prior with him. They charged me for two counts of adultery. One for the consensual and one for the rape. And I fought the rape, and then that one was taken off of my paperwork, but, by that point in time, the damage was already done. (35-year-old woman, Air Force veteran: 11 years of service)

Survivors also reported that they were afraid to report the abuse for the fear of being charged with adultery and further risking their career:

So, that was one of the reasons why I didn't originally change it from restricted to unrestricted, because I was terrified. Am I gonna lose my rank? Am I gonna get kicked out? Am I gonna get into trouble? And, you know, I'm already losing my fucking mind. And I'm gonna lose my rank and my kids. You know what I'm saying? I can't deal with this right now. (38-year-old woman, Army veteran: 14 years of service)

\section{The Normalization of MST Incidents}

A third factor contributing to the culture of sexism and misogyny is the perception of tolerance of inappropriate comments, fraternization and predatory behavior due to 
incidents of sexual harassment and sexual assault, which, when left unchallenged, often become normalized. This tolerance or indifference can show up in a multitude of ways. Consequently, a perception of tolerance for MST exists for all servicemembers, especially for the victims and perpetrators. For example, the open predatory behavior even became a joke amongst peer groups, where the complaints were often ignored and/or the person complaining dismissed. As one interviewee described, I'm brushing my teeth at the sink, and there's another girl beside me, and this dude pops his head. He's like, "Oh, I'm sorry...got the wrong bathroom.” And the girl goes, "You know, the same dude did that last night." So, I saw him in the cafeteria. I pointed at the guy like, “That dude's like a fricking creep and a weirdo.” They were like, “Oh, Peeping Tom.” I said, "What are you talking about? Are you telling me that this is a thing, and you all know about it?" He said, "Yeah, [at] the other base, the MPs actually had to bring him to the commander because they caught him hanging at the top over the wall looking into the shower." But I was just like, "You all are joking about this crap. This is a known thing. I mean, you got a nickname: 'Peeping Tom.' He's going to end up fricking raping somebody. I mean, you all are just making jokes about it, but nobody's actually taking this crap serious." (20-year-old woman, Army veteran: 3 years of service)

Additionally, this normalization of sexual violence imposed an additional burden on the survivors to keep themselves safe and prevent potential victimization. Whether serving in the U.S., overseas, or on deployment, survivors reported feeling unsafe in the 
barracks or military housing. In some situations, the (oftentimes repeated) predatory behavior was an open secret amongst rank and file servicemembers, where peer-to-peer victimization was occurring. A survivor described here experience:

Because those barracks were unreal. I mean, they make these frat colleges seem like nothing. When we showed up from reception to our unit, there was already 10, 15 guys lined up outside the barracks. Then, I started to see what was going on, that these guys were trying to get the new girls drunk and messed up on pills, so they could take advantage of them. Within 30 minutes, there were guys banging on my door. That, in itself, I felt like I put myself in that situation. That's kind of the mentality of the whole atmosphere there is, if you're going to get drunk, or messed up, then, whatever happens to you, is your fault. I saw what happened when the next wave of transports came in. They would get these girls beyond drunk, passing out off of Percocet, and they would take turns raping them in the barracks." (*35 Woman, Army veteran: 8 years of service*)

In other situations, servicemembers were victimized by more senior non-commissioned officers in the barracks, regardless of whether there was an existing personal relationship. As one participant stated,

I guess I didn't fully close my door, and he was an E6 or E7. We had flirted a little bit, but nothing like crazy. We never even kissed or anything like that, and he came in my room drunk and got on top of me...He had his hands on my

$35 *$ No age was provided by the participant 
breasts and stuff, and his hand was down my pants. Thankfully, it didn't progress to more than that because I had the phone right next to me. At that point, I realized I couldn't get him off of me, and, no matter how many times I told him to stop, he wasn't listening. He was just shit faced, and I had hit the speakerphone on my phone and hit redial because I had just been talking to one of my male friends who lived the next door down heard me saying "Stop. Don’t. Help." (40-year-old woman, Active Duty Army: 16 years of service)

Lastly, in the aftermath of a sexual assault, the gossip that would ensue within the military unit usually questioned whether the victim was drinking as well as reinforced the idea that preventing sexual violence is up to the victim instead of the perpetrator. One interviewee mentioned,

I remember if there was a specific incident that occurred, I remember a report had come out that a girl was raped. I remember gossip: "I bet she just got drunk, and that's how that happened" and stuff like that. And [when it] happened to me, it made me not want to say anything after that. (20-year-old woman, Army veteran:

3 years of service)

\section{Victims Were Often Bullied and Slut-Shamed}

Survivors also expressed concern over the bullying and slut-shaming that occurred from their peers, their leaders, the perpetrators, and the spouses of the perpetrators. These type of bullying behaviors varied among people. The most common were examples of the perpetrators punishing the victims for rejecting their advances 
and/or intimidating their victims to keep them from coming forward to report the incident(s). One survivor recounted,

I'm pretty sure he found out that I was going to tell because I was trying to get into the computer lab and Cadet Hall, and he's like physically blocked the door and just like stared me down. And then, after I reported and after he was like banned from Cadet Hall, sometimes, like at night, I would see him walking right past my dorm like by the back door at night. I had to start completely avoiding the student union center 'cause I knew that's where he always hung out. (24-year-old woman, Active Duty Army: 3 years of service)

Another form of bullying and slut shaming occurs when leaders respond to a report of sexual harassment by singularly focusing on and bringing attention to the survivor's sexuality and implied promiscuity, which is a form of victim-blaming. For example, in the following quote, this active duty Army officer recounted being repeatedly singled out by other women leaders after she reported a fellow officer for sexual harassment during her deployment to Iraq. She shared,

I was actually mandated [by leadership] to open my packages up in front of people because I guess they thought that I was ordering some sexy...but nobody else was made to open up their packages. And even to a point where I ended up getting the flu. I was so sick and they were like, "you need to take a pregnancy test.” They just assumed that I was screwing everybody. I'm like, “do you test every female that comes in for flu symptoms for pregnancy?" They're like, "No." I'm like, “So why am I being singled out?” And I honestly believe I was being 
singled out because of that whole sexual harassment thing. That started everything, and it wasn't even my fault, but yet they kept blaming me for all of it... (40-year-old woman, Active Duty Army: 16 years of service)

An additional example, that is not as common, was the perpetrator's spouse also participating in spreading rumors and bullying the victim, oftentimes reframing the incidents of sexual assault as the victim's attempt to get attention and/or seduce other married men. One survivor mentioned,

His lovely wife posted in kind a public place. It is a website where they trash people. It says that I was a liar and that my rape didn't happen, that I had an STD and that I was spreading it to everybody and I was a whore, and that I was crying wolf, and that, every time I would get into trouble, I would cry rape and that I had done this to all these supposedly married men, and here I'm the one looking like the victim when, in reality, I'm the one causing the problem. It used to be the first thing you could find once you Google my name. That website hurt me in so many different ways. Partially because it was like people would think that I'm lying about my rape, even though it's true, you know, people are going to think I'm supposedly lying, you know what I mean?" (38-year-old woman, Army veteran: 14 years of service)

\section{Theme Two: The Leadership and Chain of Command Matters}

The second finding was that leaders (NCOs, commissioned officers, warrant officers) played a key role in how the survivors processed their experience(s) with MST, their (in)ability to heal, and their overall day-to-day functioning. This is even more 
significant if said leaders were within the survivors' direct chain of command. There were four specific areas the survivors identified that served as either barriers or enablers to their overall recovery and feelings of inclusion and value as a contributing member of the military.

\section{Leaders are Responsible for the Command Climate of their Military Unit}

First, whether leaders modeled a positive command climate in their military unit contributed to whether the survivors felt comfortable reporting the MST incidents and whether they could trust that they would be treated fairly if they decided to come forward and report the incident. For example, in the following quote, the lower enlisted soldier was being continually sexually harassed by an officer while she was deployed to Iraq. As per stated policy and her training, she reported the incident to her squad leader for his help to deal with it, only for her to be blamed and her experience minimized:

So, given I felt so close to my sergeant, that evening I told him what happened. His immediate response was, "well, maybe he felt it was okay because you are very friendly. Maybe he's seen how you were and he thought it was okay. "So, that immediately changed for me because I just felt so tight with him that it really hurt. So, that immediately was like a slap to the face to me. I thought, "Well, shit, first person I'm supposed to report it to, and then that's the response. (20-year-old woman, Army veteran: 3 years of service)

There is also a perception that the command climate that is often hostile to women is an intentional part of the culture designed to minimize their contributions and their overall 
value to the military, which survivors felt contributed to the normalization of harassing and abusive behavior. As one survivor mentioned,

And the military isn't there yet, but it's way past time. I think this has been deliberate. I don't think it's been an accident because there are people who don't want women on the front line, for reasons that are not, that don't pertain to national security. That don't pertain to mission readiness. There is an ideology at play. That's what we're battling. It's ideology. (46-year-old woman, Air Force veteran: 9 years of service)

A negative command climate consisted not just of workplace sexual harassment but included incidents of sexual assault as well:

On another deployment, an officer had been being kind of weird, you know, flirting with me. He was probably mid- to late 40 s. He was actually what they call a mustang (he has been a chief ${ }^{36}$ before) and really salty. He got me cornered, had me pinned against one of my workspaces one time, and was masturbating onto me. I didn't tell anybody because it's so humiliating, you know? That was after I'd already tried to go to the $\mathrm{SAPR}^{37}$ for something that I considered was based on my gender, and he didn't help..." (38-year-old woman, Navy veteran: 6 years of service)

36 A chief is a type of non-commissioned officer (NCO) in the Navy

37 Sexual Assault Prevention and Response (Coordinator) 


\section{Inconsistent Treatment for Women Versus Men by the Leadership}

Survivors cited inconsistent standards on how leaders treat men and women concerning personal and professional interpersonal relationships. One participant stated, So, all of those situations have always been like, “it doesn't look appropriate that you're hanging out with specific guys solely on regular occasions." It gives the impression, and so that's always been their thing. That's the consistent that I've always gotten was, "it gives the wrong impression." Nothing about the guys. It was directed at me. There were accusations that I was seeking out these guys and sleeping with them. (37-year-old woman, Active Duty Army: 20 years of service)

In most, if not all, cases of fraternization and sex, the women reported that their leaders imposed more restrictions on their movement and behavior and judged them more harshly than they did their male peers. One interviewee said,

The reason why it was getting so heated between him and I on top of that is because he gave me a curfew, told me I had to be back at the tent, that I couldn't go fraternize or couldn't go talk to the air force or anybody else that I wanted to, that I had to be back at the tent. But, yet, we had five other guys that while they're over there playing cards, and I can hear their conversation, "Did you see that chick in MI, or MP. I should go do her," and stuff like that. But, yet, I got a curfew. (20-year-old woman, Army veteran: 3 years of service)

This type of action, however well-intentioned, results in further isolating the women and adversely impacting their career. The survivors shared that this type of burden was disproportionately experienced by the women, rather than their male peers. 
This segregation compounded the existing feelings of marginalization and isolation the women were already experiencing. A participant stated,

As an only female in a 12, literally, man team, I find guys that I can feel safe with, you know? And I closely associate with them, but, as a specialist, I had two lieutenants and a captain, and one in particular would always be like, "hey, you shouldn't be hanging out with the guys so much," and my question is, "well, who else am I supposed to be hanging out with? Either you select another female, or let me associate with the people I feel most comfortable with." (37-year-old woman, Active Duty Army: 20 years of service)

It is also to be expected that leaders would believe that any alcohol (ab)use by a female made MST solely the responsibility of the victim, versus viewing the MST as the criminal action of the perpetrator. Even on deployment, servicewomen are dissuaded from fraternizing and consuming alcohol with others outside their unit by their top NCOs and officers, especially unaccompanied without a battle buddy. As such, these leaders attributed subsequent crimes as the fault of the victims. One survivor recalled,

First Sergeant would frame it that these women went over to the Italian tent, these women went without a battle, they had a female battle buddy, but they were over there drinking and got drunk. And we weren't supposed to be drinking, so they acted like, if we did something we weren't supposed to, you might get raped type of thing, was how it came across. It's getting the male command to look at it differently. (35-year-old woman, Army veteran: 4 years of service) 
In response to the incidents of fraternization, adultery, sexual harassment and sexual assault that would occur, the participants shared that their leaders often resorted to restricting the movement and activities of women servicemembers as a strategy to prevent the incidents.

Additionally, survivors shared the isolation they experienced when they were subjected to "all or nothing" professional encounters where they were either ignored or sexually harassed by male servicemembers. This is often the consequence of prevention strategies that rely on men avoiding contact with women altogether so as not to risk offending or being accused of victimizing them, versus finding a way to professionally engage will all servicemembers, regardless of their military occupational specialty (MOS) or gender.

The people that were the scaredest to say anything as far as sexual harassment and all that stuff were the infantry. I guess because it was ingrained in them: "Don't you say a thing to any of these females." It's-going-to-be-the-end-for-you-type thing of you just can't." I know that only because I spoke to some in [location of deployment], and then they were like, "I'm still, I'm not supposed to speak to you." They didn't even want to be my friend or anything because they might offend me. (20-year-old woman, Army veteran: 3 years of service)

\section{Survivors Experienced Professional Retaliation by Leadership for Rejecting}

\section{Advances and/or Reporting Incidents}

There were also multiple examples where the survivors experienced professional retaliation for either rejecting sexual advances or reporting incident(s) of sexual 
harassment and sexual assault. In some instances, survivors shared that they were second guessed when reporting the incidents, often times being threatened with UCMJ disciplinary action related to the incidents. A participant recalled one such incident:

Every time you go up the chain, you have to explain the whole thing again. Every single time you explain it, you get the same damn thing: "We're going to piss test you. Are you sure you want to go this far? You were underage drinking? Are you sure ... Oh, you don't remember all of them, so you're not quite sure if it was 'Smith' or 'Jones?' You saw them there, but are you sure that they were the ones that did this? You're not really sure, because you were passed out, so do you really want to go through this? Because if we find out, through witnesses, that they weren't involved in it, then, you're the one who's going to get in trouble. You're the one who's going to get UCMJ.” It was every single step. (*Woman, Army veteran: 8 years of service ${ }^{* 38}$ )

Another example of professional retaliation by leadership was withholding promotion and advancement opportunities from the victim:

MTLs (the) military training leaders, they became really close with him. And he was married. They believed him. There were many people that didn't believe me. But I even wanted to be in a leadership program. Tt's called leadership ropes, and I was told by the head person that, uh, because I accused him, they wouldn't let me be a rope. And so, I went around that girl and became a rope anyways, so then 
I ended up graduating with honors from my tech school. (31-year-old woman, Air Force veteran: 6 years of service)

Survivors also described the additional trauma and betrayal they experienced when they were ignored and disbelieved by their leaders, oftentimes feeling further endangered and victimized. As one survivor said,

He kept coming after me, and I kept blowing him off. I guess he got jealous, he grabbed me, slammed me against the wall one day, pinned me to the wall, and was screaming in my face, saying that I was his, and that I guess, he thought that I was. He said, "I saw you kissing somebody else, and blah, blah, blah.” And yeah, it got real nasty. I mean, I was crying, and where I had slammed my head against the, it was a brick wall. And I had told the Sergeant major, who told the Commander and I don't know who else was told, and then nothing happened, but he did kind of back off, so I don't know if they talked to him. He backed off from me, but then he started. He didn't get kicked out, until he got caught in our barracks with another girl, and that kind of pissed me off. I mean, I was happy that he was gone, but it still kind of pissed me off, I'm like, "great, he slammed me up against the wall, and nobody does shit, but he gets caught screwing one of the girls, and you guys are like, oh you're gone now." (40-year-old woman, Active Duty Army: 16 years of service)

Incidents like these become even more problematic when the unit leadership takes an active role in dissuading the victim from moving forward with reporting the incident, oftentimes citing the potential for them to lose career opportunities and the likelihood of 
the charges not sticking for the perpetrator. These type of behaviors on the part of the unit leader contribute to the sense of MST being tolerated and the futility of reporting the incident(s). One interviewee stated,

Yeah, because they told me that, if I did unrestricted, to press charges of any kind, that it would be on a Page $13^{39}$, and it would follow me for the rest of my life, my service folder. And he [the commander] also told me specifically that charges like that rarely get successfully prosecuted. You know, something like less than ten percent. And he said, 'so you're going to make charges against this guy and it's not going to stick"” (38-year-old woman, Navy veteran: 6 years of service).

\section{The Career of the Perpetrator is often Prioritized Over That of the Victims}

Fourth, survivors shared several examples where the careers of the male perpetrators were given more consideration and priority, than that of the female victims' careers. One survivor said,

[It] didn't matter that I had an impeccable career up until that point. [I] was always willing to help out or do whatever they needed to do. But the second [that] something bad happened to me, I was trash. (33-year-old man, Army veteran: 2 years of service)

Survivors discussed feeling frustrated and angry that, even after their MST incidents were reported, the careers of their perpetrators were either not impacted, or, worse, they were promoted and were able to advance their careers. In situations involving

39 Page 13 is a type of administrative action (positive or negative) that is included in a sailor's personnel record in (Navy Military Personnel Manual, 2019) 
sexual harassment, oftentimes service members were given the message (directly or indirectly), that reporting such incidents would damage the perpetrator's career, further underscoring the message that such behaviors are tolerated, and, perhaps, that it is neither recommended to report sexual misconduct nor intervene on someone's behalf unless a servicemember wants to draw the same speculation or ire from their peers. One interviewee recalled,

During bootcamp, I remember a girl was getting harassed by this other enlisted, and I remember her speaking up about it, and the drill sergeants just separated her because they talked, and I remember hearing that "You can destroy somebody's career." That reinforced me to keep my mouth shut because I didn't want to have to go through saying something about it and then being reprimanded for it. If someone came around and needed my comfort, I would give it but I didn't go out of my way. (42-year-old woman, Army veteran: 1 year of service)

\section{What a Positive and Supportive Military Environment Looks Like to a Survivor}

In contrast to the negative leadership experiences the participants shared, there were five areas that they felt contributed to a positive and supportive military environment, especially in the aftermath of MST. First, survivors' feelings of support and inclusion had less to do with gender stereotypes being explicitly challenged or defending women's equal place in the military like their male peers. Instead, survivors wanted to know someone else "has their back," can be trusted and will stand up for them, as their male peers enjoy with other male service members. One survivor mentioned, 
He [perpetrator] was tight with one of the infantry guys that had a tank. Anywhere they went, he rode with him in their thing. Well, I was close with that sergeant and told him what had happened, and he came to me and goes, "I want you to know that, yesterday, he tried to climb up in that tank, and I told him he was no longer welcome and he just had this look of fear on his face, like he could lose his life at that point without being no longer in his tank." So, [I] felt a little better about that. (20-year-old woman, Army veteran: 3 years of service)

Additionally, survivors felt that a supportive environment was made possible by their leaders modeling the type of trust where the survivor not only felt safe enough to confide in, but validated their overall strength and resiliency.

It was my command here after the actual court martial took place. He was the first person. It was my chief of staff, my troop commander, said, "Listen to me, what you went through does not define you." And that was really the first time that I thought, "holy shit, it doesn't." (41-year-old woman, active duty Army: 19 years of service)

Second, the participants described that a healthy environment exists when survivors feel empowered and, more importantly, are encouraged to report the MST incidents by their peers and leaders who also support them throughout the process. One participant recounted a conversation with a leader: 
I told this $\mathrm{E} 7 \mathrm{NCO}^{40}$ who just, like I said, we just happened to be friends. We were semi-trained to get prepared for $\mathrm{NCT}^{41}$. And, somehow, I just confided in him, and I said, "I got raped." And he just looked at me like, "you need to say something," and I told him, "I promise you, I will." (38-year-old woman, Army veteran: 14 years of service)

Third, survivors shared feeling supported and less victimized by their leaders when they were given access to resources right away and felt heard by their leaders, basically fulfilling the promise they were given in the prevention training and in what was communicated to them by their chain of command. One survivor recounted, I went unrestricted ${ }^{42}$ in September. And at that, by the time I did, I had the $\mathrm{SARC}^{43}$ helping me. I was going to mental health and they were - the mentalthe therapist or psychiatrist, I don't remember what she was, was amazing. And I had gotten a special victims counsel because I was going unrestricted. (39-yearold woman, Air Force veteran: 22 years of service)

Fourth, a supportive climate is reflected by leaders who provided both the space for the survivor to confide in them as well as the resources, tools, and, most importantly, the time to heal properly while concurrently assuring them of their continued value and worth to the unit. One interviewee stated,

40 Non-Commissioned Officer usually senior sergeants in the enlisted corps and in key leadership positions 41 National Training Center (NTC) at Fort Irwin, California is an Army readiness center for desert warfare 42 Unrestricted reporting refers to the option sexual assault victims to formally open up an investigation while receiving support 43 Sexual Assault Response Coordinator (SARC) 
I went to my then-commander (this is my third commander since I've been here), and I told him what was going on. He was like, "Wow, I never would have known that. Listening to you and knowing you, so I thank you for sharing." And, so, he cut back on my duties, and he was supportive, and I ended up going to an outpatient facility for several months and then an inpatient unit and then going through the med board process. (39-year-old woman, Air Force veteran: 22 years of service)

Additionally, survivors identified positive environments as ones where their leaders are sensitive and attuned to their subordinates needs. For example, another survivor stated,

So, it took moving to a place where I'm by myself, but I had good leadership. I still had lots of issues, but the officer that became my supervisor when I got here knew me from before. He was supportive, but he wasn't intrusive. He knew what was going on, and he provided me the environment to be able to do my job the best I could in the situation I was at. And he trusted me. He had faith in me. He allowed me to do what I could, when I could, how I could. And that gave me more energy and strength to do it because I want to do my job. I want to show him that his faith is not misplaced, his support is not misplaced. It gives you a boost. Instead of having somebody who's like a dictator or micromanaging, like that just stresses you out and debilitates you. Where, if they empower you, you do better, you succeed. (39-year-old woman, Air Force veteran: 22 years of service) 
Fifth, the survivors who felt the most supported and validated shared that their mental health professionals helped them understand their trauma and symptomology connected to their sexual assault versus being subjected to labels and diagnoses. The normalization of their reactions and experiences by their mental health counselors helped them process their reactions and experiences in a less isolating and judgmental way. One survivor recalled,

Yeah. I don't know if it was borderline. I forget which one it was, honestly. I don't have a personality disorder. I was having a very common trauma reaction, so, I mean, for them to classify as something else. I remember my psychiatrist who I was seeing as a civilian. He's like, “I've been talking to you for two years. I know you don't have a personality disorder. This is context trauma. It's kind of textbook." (38-year-old woman, Air Force veteran: 7 years of service)

\section{Theme Three: Response Efforts Were Often (Re)-Traumatizing}

In this section, survivors described the ways their MST experience(s) impacted

them physically and psychologically, as well as how they were treated by the military and Veterans Administration Hospital when they sought medical and counseling treatment. Additionally, LGBTQ survivors identified the additional stress of reporting the MST when they served during the "Don't Ask, Don't Tell” era.

\section{MST Has a Lifelong Impact on the Survivors}

First, survivors shared the totality of the personal impact the MST incident(s) had on them. Common effects include feelings of alienation and social isolation, often coexisting with concurrent mental health issues and substance abuse. Survivors often coped 
with the trauma, stress and isolation by resorting to self-destructive behaviors such as cutting and/or eating disorders. One survivor stated,

It didn't seem to matter to anybody, and, because of how much it mattered to me and just how devastated I was and not knowing how to cope with that, still having not learned effective coping mechanisms, I remember sitting at home one night in my apartment and I just started cutting my arm and I had never thought to do that before and it's not something I had ever seen anyone else do. I just remember thinking and feeling that I needed some sort of physical manifestation of this internal pain that I had because nobody seemed to understand how much it hurt me, and I was like, "you know what? Maybe this will make it visible." And that, of course, landed me in the psych ward and gave them all the ammunition they needed. (38-year-old woman, Air Force veteran: 7 years of service)

Consequently, these adverse experiences compounded the victimization and trauma, often leading to feelings of distrust, depression, anxiety, substance abuse and potential self-harm. Several survivors shared that they attempted suicide, sometimes multiple times because of the totality of the isolation, workplace stress, and sometimes bullying they received from their unit after they reported the MST incident(s). One survivor stated,

I have no safety net to protect me from talking. So, I just didn't talk. And, instead of talking, I tried to kill myself. Instead of getting any counseling for the fact that I had just been raped, they put me in a locked, padded room for two weeks with no social interaction. There was no safety. I had no safe space. I didn't, you know, 
I journaled, but that doesn't replace human interaction, not when you're suffering and you don't understand why nobody's talking to you. So, needless to say, [I] attempted suicide. Um, shortly after my assault, when I reported the assault, I was in the hospital for a while, got out of the hospital, did not reattempt suicide. They said I did, put me right back in that lockdown unit, and served me my discharge paperwork while I was in the hospital and subsequently discharged me. I got out of the hospital. I was out of the hospital for one day and I was out-processed of the military that day with a preexisting borderline personality disorder, is what they diagnosed me as. (33-year-old man, Army veteran: 2 years of service) The prospect of damaging or losing their career entirely was an ongoing (and legitimate) fear for many of the survivors, leading to avoidance of mental health treatment. They noted that certain mental health diagnoses could mean forfeiting their full military benefits (medical, educational, housing, etc.), particularly if it could be proven as a pre-existing condition to their military career. They had to balance the prospect of one lifelong impact on their career against the other lifelong impact, such as untreated mental health issues and trauma. One survivor named it the nuts and sluts. You know what the protocol is. You give them the personality disorder diagnosis. You give them the, you know, whatever bullshit psychiatric diagnosis. You completely disregard anything else they say because obviously they're crazy and then you administratively push them out as quickly as possible. (38-year-old woman, Air Force veteran: 7 years of service) 
Survivors often put off or avoided accessing treatment as long as possible, to the detriment of their work and overall mental health because of the added stigma of being labeled with a mental health disorder, and possibly being kicked out of the military. One survivor recalled,

I drew it out, um, as long as I could, the best I could. I had to work extra hard to do the same amount of work that I was doing before. At work, I was excelling in people's eyes, but I knew the truth, which I'm doing everything I can to stay afloat on the weekends. I wouldn't get out of bed, but my family was suffering. Like, for two years, I was keeping my career afloat, but at a severe cost to myself and my kids. Life ceased and desisted as we had known it before this. I'm positive that the fall of 2013 was the downfall of my career. (39-year-old woman, Air Force veteran: 22 years of service)

\section{Survivors Were Often Treated Poorly by Military and VA Health Care Providers}

Second, the majority of the survivors' feedback about the response efforts focused on how they were treated by either the military's healthcare system while on active duty, or the Veterans Administration (VA) healthcare system upon their discharge. For example, one participant mentioned,

When I searched for counseling, the first lady that I spoke to, she told me it was my fault, so I hesitated to even mention it the second time around. You know, but it just felt like one let down to the other. (38-year-old woman, Army veteran: 14 years of service) 
Some participants shared that when they reached out for medical attention after the sexual assault, they were subjected to victim-blaming instead of being supported in their healing.

It was a hospital commander. He was a full bird colonel, a psychiatrist. He was the one that told me that was inappropriate attire. I shouldn't be wearing things like that and that's what gets men to do what they, you know, whatever. (40-yearold woman, Active Duty Army: 16 years of service)

This victim-blaming also occurs to some survivors who experience sexual violence from their servicemember spouse when seeking support and treatment for their rape and/or domestic violence. One participant recalled, It was a conversation about, well, the way I addressed it was with my sex drive and I have like no libido, so I agreed to have behavioral health counseling. It was on post ${ }^{44}$ at the Behavioral Health Clinic, and the first and only appointment that I participated in was with a male, and he basically just accused me of not wanting to give my husband BJs [blowjobs]. This was in our intake appointment, and I was just so mortified, and I never stepped foot in that building again. (44-year-old woman, Army veteran: 4 years of service)

Other survivors shared that, upon sharing their sexual harassment and/or sexual assault experiences with mental health counselors, they felt disbelieved, minimized or attacked

\footnotetext{
${ }^{44}$ Post is an Army term to refer to the military installation like the term "base" would be used for an Air
} Force installation. 
by the professionals they were told to turn to for protection, support and accountability.

One participant recalled,

I remember I went to her office and I had been seeing for about a year for my PTSD and hadn't really ever opened the floodgates, and I decided, "You know what? I trust his lady, I've been seeing her for a year, I think I'm ready to tell her what happened to me.” In my backpack, I had my rape report. I had it with me. And I started telling my story and she said, "Stop.” And I said, "What?" She said, “Stop lying to me." And I'm like, "What the fuck are you talking about? I'm not lying to you." And she said, she said, "You were molested in high school. You were never raped in the military." And I said, "I was raped in the military on this date, this time, here's my report." She took my rape report and ripped it in half, and I took her desk and flipped it and told her to fuck off, so, the one time I tried to talk to somebody about it, I was called a liar. (33-year-old man, Army veteran: 2 years of service)

The survivors who identified as veterans (versus active duty servicemembers) cited multiple cases where the VA hospitals were unprepared to treat victims of MST in terms of subject matter expertise and enough personnel. One survivor shared,

When I first started my VA care back in 2006, late 2006, some physicians at the VA, they don't know what MST is because I have this new doctor at the VA here at the clinic, the outpatient clinic, and she asked me what is MST? I looked at her and I reminded myself, “Okay, she's new. She's a civilian. She came from private practice. She doesn't know." But, had I been one of those distraught women that 
have just had all kinds of shit in her life, I would've like lashed out, but then I thought about it and it kind of did bother me, but I'm like, "Oh, well, I told her maybe next time you have a patient and it says that, don't go, "Oh, well, what is this? But then that's the VA, like you said. Their orientation, who knows what it's like? (42-year-old woman, Army veteran: 1 year of service)

Additionally, a lot of survivors complained they were unable to get appointments at the VA Hospitals, even if they were in crisis. Oftentimes the default or short-term solution was to give them medication while they wait for the next available appointment.

When I contacted the VA, they're like, "Well, when would you like an appointment?" I'm like, “The first available,” and he's finally like, “Look, the first available is in a month." I'm like, "I'll take it." But a month when a person is in crisis, it's too long to wait. That's not talking to the psychologist, or the therapist. That's just getting an intake, so I can get the referral to talk to that person. From there, it was another month. The whole time, I'm in crisis, and then their first response is, "Let's put you on some medication. It'll make it all better." And it's like, 'No. That's not, that's not what I need. I need skills to fix this, I don't want to be medicated, I want skills to make this better. To work through it, or whatever. I need skills.” And they were really reluctant on the skills. I really had to advocate for myself in order to get the cognitive thoughts processing, and all of that stuff. That's the other part of it, removing those barriers. Somebody in emotional crisis. We're having too many suicides, and a lot of it is because they 
can't get the care when they need it. They have to wait. (35-year-old woman, Army veteran: 4 years of service)

\section{Discriminatory Policies like “Don’t Ask Don’t Tell” Can Deter Reporting Incidents of MST}

A specific source of stress and isolation shared by the LGBTQ survivors was the existence of punitive policies like “Don’t Ask, Don’t Tell, Don’t Pursue” (DADT). While this policy, in theory, was supposed to protect the careers of LGBTQ servicemembers by not forcing them to disclose their sexual orientation or gender identity, it became problematic for LGBTQ victims of MST who came forward to report the incident and engaged with the military justice system. The DADT policy allowed for the servicemember's privacy, but it did not totally shield them from being targeted or harassed by leaders or military defense lawyers. As one participant discussed, I identify as a lesbian. I was not out then. The defense [attorney] brought me in because I served under Don't Ask, Don’t Tell. So, they asked me if I was gay, and I told them I could not tell them that. They said, “well, the legislation's about to pass, and it's gonna be able to be lifted." And I said, "I can't tell you anything and you can't ask that. They had started the process of investigating me for being gay in the military, so I had to get a attorney at a different base because. Obviously, at that base, they were his attorneys, so I had to call a different attorney at a different base to ask what I needed to do. And they told me that I needed to plead the Fifth if they ask me any of those questions. And, so the Article 15 hearing happened and he ended up being found not in violation, and there was no sexual assault that 
happened, even with the wire admission. It was really hard because I felt like I could not tell my story to the truth because, if I told the air force attorneys that I was gay and that's why I would not sleep with him, they would have prosecuted me if, just like Don't Ask, Don't Tell was repealed. And so, I was at a loss either way. (31-year-old woman, Air Force veteran: 6 years of service)

\section{Theme Four: Prevention Efforts, In Particular Training, are Important and Often Inadequate}

The majority of the military's prevention efforts are grounded in training, and survivors trace the inadequacy of these efforts to three key areas. The military's limitations in the areas of training content, inconsistent delivery, and the skills of facilitators result in the perception that the military's efforts are not taken seriously by the servicemembers or prioritized by the leadership and chain of command.

\section{The Perceived Quality of the Training Content}

First, the content of the prevention training was an area that survivors reported feeling was disconnected from the reality of their MST experiences, an event that felt like a "check the box" exercise. Consequently, whatever information was communicated often was not taken seriously by the servicemembers. As one survivor mentioned, In the video, the guy comes up and reaches a hand out and grabs a boob or something. That's not really how it happens, and so the videos actually made it seem worse to me. You spend the whole time with your peers in the back making fun of it, you know? Especially before something happens. And then, even after 
something happened, you realize that it's never as cut and dried as it is in the videos, you know? (38-year-old woman, Navy veteran: 6 years of service) It becomes especially frustrating for servicemembers who have survived sexual harassment and/or sexual assault to sit through training that directly contradicts how they or their peers were treated, especially after reporting the incidents. One survivor described it as a joke:

I remember watching a video, and they're like, "we have no tolerance for sexual harassment, speak up, stand up, say something." And I'm like, "You're full of shit. There's nothing accurate about this video, you know? And I can guarantee you that all my other people I served with and people that I know post-military that were sexually assaulted or harassed, I mean, it's not accurate. The Army doesn't do anything for you if you report it. (33-year-old man, Army veteran: 2 years of service)

The survivors shared that, in addition to the unrealistic scenarios of sexual harassment and sexual assault, the explicit information detailing reporting options and the process were not always included. This discrepancy would often result in survivors being dependent on the knowledge and/or the will of their unit leader to make them informed of their reporting options, and what resources and support were available. As one survivor recalled,

I had already had previous sexual assault experience. There were parts of the training that, yes, they were fine, but there were still parts that were kind of iffy in my mind. Never did it tell you that you can report two different statuses of 
unreported or reported. It never goes into “this is what's gonna happen when you report." I didn't learn that, nor did I learn that if you told somebody about it, it would have to go into a reported status until way after I had reported. (35-yearold woman, Air Force veteran:11 years of service)

\section{The Delivery of the Information Matters (ex. Timing, Methods, etc.)}

Second, the delivery of the military's policy and training is another area of deficiency that may adversely impact MST prevention efforts. Military personnel are supposed to receive regular scheduled MST prevention training from the beginning and throughout their career. However, when participants were asked about the first time they heard about the military's policies towards sexual harassment and sexual assault, their answers were that it was usually via a formal training session in the early stages of their career, and, depending on the prioritization of the military unit, it was or was not scheduled regularly and/or repeatedly, such as on a quarterly, semi-annual, or annual basis. One participant said,

I don't remember hearing anything about it during my first enlistment. Like, for the first three or four years, at most the only thing I heard about it was "zero tolerance for" and they would say "sexual harassment, racism, prejudice" like they were putting everything together... like zero tolerance for these things. That's the only thing I can recall hearing before SARC came along. (39-year-old woman, Air Force veteran: 22 years of service)

A variety of prevention training formats were used with differing implications for the experience of service members, especially for those who have survived MST. 
Formats range from using sole computer-based programs to using an on-site facilitator to deliver the content, or a combination of both. There were mixed reviews about which formats survivors preferred or found most effective. On one hand, the computer-based programs provide a format to deliver relevant information in a "safe" format for survivors. However, this format includes no discussion or engagement from attendees, so it is difficult to gauge if and how much the servicemembers connected with the information presented. As one interviewee stated, If you were a victim in the auditorium, you didn't dare say anything or raise a hand or ask a question or anything. If you were a victim and you were watching these video trainings, then you could listen to what they're saying, replay the parts that you need replayed to know how to protect yourself or at least report it. (58year-old woman, Army veteran: 33 years of service)

On the other hand, in-person training delivered by instructors provide a format to deliver relevant information and, depending on the skill level of the facilitator, an opportunity to engage the service members in a meaningful way.

So, originally, it was PowerPoint, and there was somebody up there speaking. And then, at one point, it changed to the online. And then, a few years, God, I can't remember when. It might've been a while now. Then, it was you had to do the online course and the in-person course. And, about a year ago, they changed it where we don't have to do the online anymore, but it has to be face to face. (40year-old woman, Active Duty Army: 16 years of service) 


\section{The Skills of the Facilitators are Key}

Third, on-site instructors have the advantage of collectively engaging training participants in ways missed by individual computer-based training. However, depending on the instructors' facilitation skills in handling awkward and difficult subject matter like sexual consent and other MST prevention-related topics, an on-site instructor may prove to be more damaging in terms of communicating competence and safety to an audience of potential survivors of sexual harassment and sexual assault. Respondents named a range of inadequate skills that hindered a facilitator's ability to communicate effectively. Specifically, survivors identified two problematic scenarios. One scenario was being overly dependent on a video to communicate the message without a follow-up discussion to engage participant questions. For example, one participant remembered,

I think my cadre was being more reactive than proactive. I think they were probably uncomfortable with the situation and didn't really know how to go about it. And I think they somehow, wherever, got a hold of this video and they're like, “Okay, well, let's show the kids this and maybe like a message will hit home." And it was very much more of like, “Don't, basically, don't touch people. Drunk people can't give consent." There was no like really active conversation or dialogue about it. It was more just, “don't do this," and then that was that. And we didn't talk about it for another year. (24-year-old woman, Active Duty Army: 3 years of service)

Another scenario involved the trainer or facilitator making fun of the content or allowing the service members in attendance to make inappropriate comments, either because it 
makes them uncomfortable or they did not see the value of the training itself. Either behavior creates an unsafe environment that lets the servicemembers attending the training know that MST prevention is not a military priority, much less a topic that is taken seriously. One participant described,

They laugh at it. They make jokes about it. And, yet again, the chicks that are serving and sitting right next to them just have to sit there and bite their tongues because the second they say, "hey, this isn't a joke, this is serious," oh, yeah, it's serious. We're all rapists. Yeah, we're rapists. The second you regretted it. It's like there's been this backlash, just like in politics, of, rather than listening to it or being open to new ideas, they shut it down and then they start tearing it apart. (38year-old woman, Air Force veteran: 7 years of service)

Conversely, the facilitators that seemed most effective in conducting training were attuned to their participants and customized the content and delivery according to their audience, such as in terms of type of military occupation, and modeled concern and being seen as accessible and resourceful. One participant stated,

He noticed I was distant in class and could tell that something was going on. And, on a break when everybody went outside to break, I stayed in the class 'cause that's where I felt safe. He came and talked to me and kind of just hinted at, if this was what was wrong, this is what I should do and how to do it. (39-year-old woman, Air Force veteran: 22 years of service) 


\section{Recommendations from Survivors}

In this section, survivors identified eight specific areas where military leaders could be more intentional to improve their MST prevention and response strategy, as featured in the below table.

Table 3

Recommendations

\begin{tabular}{|l|l|}
\hline Theme Area & \multicolumn{1}{|c|}{ Proposed Recommendation } \\
\hline Culture & Don't use gender segregation to prevent MST \\
\hline Leadership & Lead by example (hold leaders to high standards of professionalism) \\
\hline Leadership & Increase the consistency of accountability \\
\hline Response & Transfer investigative authority outside the Chain of Command \\
\hline Response & Treat MST as seriously as any other injury \\
\hline Response & $\begin{array}{l}\text { Improve medical/psychological support protocols for Military } \\
\text { Servicemembers and Veterans }\end{array}$ \\
\hline Prevention & $\begin{array}{l}\text { Expand MST prevention training content for servicemembers and } \\
\text { leaders }\end{array}$ \\
\hline Prevention & Improve the quality of the staff / trainers/ facilitators \\
\hline
\end{tabular}

\section{Recommendation 1: Don't use gender segregation to prevent MST}

Survivors suggested leaders allow service members to socialize with the servicemembers they feel most comfortable with, instead of imposing restrictions to which they do not subject their male peers, especially as a preventative strategy to mitigate incidents of fraternization, adultery and MST. One participant provided a recommendation: 
As a leader, if a soldier feels comfortable with another soldier, particularly women, like the female soldier will seek out the male soldier that they feel most comfortable with. Don't force them to associate with people that they don't feel comfortable with. Because, generally, they're going to migrate. They're going to cling on to the person or persons that they feel most protected around. Particularly in a scarce environment where they don't, whether it's two females or not, you don't make two females associate with each other because females don't always get along with each other. They're not going to hang out with a male that they are threatened by, whether it's by their communication methods, you know, always talking sexual harassment, stuff like that. Whether it's directed towards them or elsewhere, but I think women will naturally migrate toward the men that they feel most comfortable with, and I don't think that, as leaders, we should judge them as I have been judged in the past. I don't think that they should be judged as that looks inappropriate. Remind them about appropriateness, about proper places to be, but just to keep a keen eye out on them. (37-year-old woman, Active Duty Army: 20 years of service)

\section{Recommendation 2: Lead by example (hold leaders to high standards of professionalism)}

Another recommendation was that commissioned and non-commissioned officers were responsible for establishing and reinforcing a positive command climate, wherein every servicemember is held to the same high standard of personal and professional conduct. Survivors point to crude language and harassing behavior contributing to a 
culture that normalizes this type of conduct, especially if left unchallenged by leaders. The normalization also has the potential to be internalized by service members, many of them MST victims themselves, who either participate in the same type of behavior or fail to challenge the conduct. As one interviewee stated,

We've got to figure out a way to be able to make sure that our culture is supported by everyone having the same beliefs, professionalism, and all those things. My career field in itself is male dominant and you have to learn to kind of deal with some of the things. So, I kind of got a thick skin, and maybe I'm also to blame for the culture because it's just kind of how they are and how they behave, and, unfortunately, that's not the right answer. (40-year-old woman, Active Duty Air Force: 23 years of service)

Survivors also described a positive command climate which looks like leaders prioritizing the same time and resources as well as focusing on executing quality MST prevention training and response programs, as they do with other parts of mission readiness like physical fitness, firearms qualification, and specific job training. One survivor offered,

It depends on your leadership and how much they are willing to make it a priority. So, you'll see other bases, they might be failing at it. If they put their money where their mouth is, and they put the right people in the training and they provide them really good trainers and people that are going to have time to follow up with them and make sure that they're sending the right message because, let's face it, you teach somebody something, but if you walk away and not ever watch 
them do it again, you have absolutely no idea whether or not the message is being conveyed. It's not just about teaching people and getting a bunch of people in the room, it's about following up and making sure that the people that are providing this training are actually doing the right thing. (40-year-old woman, Active Duty Air Force: 23 years of service)

\section{Recommendation 3: Increase the consistency of accountability for perpetrators of MST}

Survivors recommended that the military promote deterrence and ensure a safer work environment by increasing accountability for perpetrators of MST, not by blaming the victims. Survivors recommended equal accountability and justice for MST incidents, regardless of gender or rank, especially if they are a high-ranking commissioned officer or an NCO. One survivor said,

But, yeah, this MST. I don't think it's ever going to stop. And it's nothing the government, yes, the government has a lot to do with it when they hide it when it's the higher officers and stuff when they all do it, but it's beginning to crack down. If you start seeing it as there's a work in progress, it's starting to change. For the better. I think it is. I mean, I'm not political, but I do see some changes going on, but it's going to take a lot. I don't think it's ever going to go away. (42year-old woman, Army veteran: 1 year of service) 


\section{Recommendation 4: Transfer investigative authority outside the Chain of}

\section{Command}

Survivors suggested that the sexual assault cases be taken away from the commander's discretion and immediately referred to the Judge Advocate General (JAG) ${ }^{45}$ because of the concern that their unit commanders had the authority to investigate incident(s) of sexual violence and over whether the cases should go to trial. This concern is grounded in the belief that their commanders lack the legal and investigative expertise and, depending on the commander's relationship with the victim and/or perpetrator, would be unable to remain objective and fair in the final outcome, especially if the commander is also the perpetrator. One interviewee mentioned,

Commanders got to say if it was a trial or not, like if there was enough evidence. I just couldn't fathom how could you put that on a commander? I mean, how are they able to make that decision? So, I know that there was mention of that being taken away. To me, it seems like it should be the legal office's determination. (39year-old woman, Air Force veteran: 22 years of service)

\section{Recommendation 5 Treat MST as seriously as any other injury}

The impact of MST, to include the physical, psychological and social effects, are often an afterthought and not treated as seriously as more conventional physical injuries that servicemembers incur during their tenure. As such, survivors recommended that it would be helpful if their leaders would treat MST as seriously as they would any other injury, which has explicit implications for leaders in terms of following up and providing

$45 \mathrm{JAG}$ is the corps of military lawyers 
access and time for MST survivors in their unit to heal properly. There are three specific ways leaders can treat MST as seriously as a conventional injury

First, consistent with the message of prioritizing healing and empowering survivors is to expand programs that are survivor-focused and trauma-informed. Programs like Wounded Warrior treat sexual assault as seriously as any other serviceconnected injury, such as a loss of "life, limb or eyesight." These types of programs allow the servicemember to heal in a therapeutic environment while protecting their career. Upon successful completion, servicemembers are allowed to return to active duty versus being discharged from the military and losing their career, and possibly their benefits. One survivor recounted,

Wounded Warrior Battalion didn't exist when I got hurt in the military, and I look back and so much of what the Army did to me could have been fixed. I met with soldiers who were part of the battalion, who were military sexual assault survivors and they weren't getting discharged for bogus reasons. They were getting counseling, they were getting treatment, they were getting help, y'know, and they were going to return to duty. That didn't exist in my era. (33-year-old man, Army veteran: 2 years of service)

Second, it was recommended that their leaders check in with the survivors in their unit, especially during the reporting process and in the aftermath of their healing. Survivors described two instances where checking in with them would be the most beneficial. 
Checking in means that the leaders and trainers/facilitators should be paying attention to survivors within the context of prevention training, which can be triggering for many servicemembers due to previous trauma incurred in the military and/or their time before their service. Survivors recommend that facilitators be trained to identify service members who may be struggling with some of the MST training content during or after the event. Furthermore, a survivor who has served as a SARC trainer in her unit spoke to the benefit of her experience informing her increased sensitivity to servicemembers displaying signs of distress during a training presentation. She recommended that an on-site training presentation would benefit from having more than one trained facilitator or leader in the room to help pay attention to the emerging needs of the service members during the event (ex. to talk, resources):

They need to have extensive training of being able to identify those that get affected by even just the talk. If I'm giving a presentation, I want to make sure that I have enough staff [to] identify just someone in the audience or someone that I'm talking to in their group, that I have one of my staff members immediately be there on the spot for that person or offer to go into another room and talk about it because you do have a lot of those people that do go out to trainings and that have been victims themselves and they just don't know quite yet how to step out of it. I think it makes it a little bit more comforting and better and a safer platform. That and just having enough staff to be there. (42-year-old woman, Army veteran:1 year of service) 
Additionally, in the aftermath of an incident of sexual harassment and/or sexual assault, a survivor wanted a leader who was supportive rather than judgmental and who is willing to meet them where they are in their healing process:

How can you help them? Not react to the actions that you're seeing, but respond to them? Meaning don't listen to respond [and] to say what you want. Like, find out what they need. How can you help them, not do what you think is best from a military supervisory accountability standpoint. Your accountability is taking care of that person, making sure they're alive every day to show up and to do what is needed. And making sure that are capable of doing what is asked of them every day. So, you got to give them the tools to do that. You gotta take care of them. I hear a lot of, you know, it's not just about the mission, like your mission is your people, but, people, we don't. It's not happening. People are still about, all about, the mission? You need to be here at work and you need to be doing this, you need to be doing that. (39-year-old woman, Air Force veteran: 22 years of service) Third, the survivors shared that a key part in their ability to heal was the followthrough of the resources and support they were promised in the official policies, consistent with their actual lived experiences. Survivors recommended improving the ability for victims to unconditionally access resources, services and the time for healing and recovery in the aftermath of their sexual assault. One survivor recalled,

I went unrestricted in September and, by the time I did, I had the SARC helping me. I was going to mental health and they were - the mental - the therapist or psychiatrist, I don't remember what she was, amazing. And I had gotten a special 
victims counsel because I was going unrestricted. Going through SARC and then going to mental health was good, was great. It actually helped me a lot. (39-yearold woman, Air Force veteran: 22 years of service)

Other examples of support included having robust advocacy, being believed by military mental health counselors, and having their expectations of the reporting process managed. As one participant stated,

They reached JAG, and they told me exactly what I was allowed to say to the commander, which is what I meant when I said that I did get a pretty interesting rare opportunity to see how my commander would deal with it. I think I was handled very well with regards to the actual MST. (36-year-old woman, Army veteran: 4 years of service)

\section{Recommendation 6: Improve medical/psychological support protocols for military servicemembers and Veterans}

Survivors recommended strengthening the medical and psychological support protocols for military servicemembers and veterans in the aftermath of the MST incident(s). The concern continues to be that the military culture and warrior ethos is still more aligned with the "suck it up" mentality, which is essentially antithetical for survivors to feel empowered to access needed services.

The survivors cited connecting with other MST survivors as helpful to processing their experiences and overall trauma. A lot of the study participants talked about being part of other MST victim groups through the VA, which helped keep them grounded and connected in their communities. 
When it was a process of getting off all of the pain killers that I had been on for the past two years and I was like okay. I'm completely sober now. I'm not drinking, I'm not taking pain medication, so I'm having to face feelings that I haven't felt since all of this shit happened in the Air Force because I have been actively, in one way or another, going out of my way not to feel them since then. So, I just really had that realization of it wasn't me. It wasn't my fault. When I finally was able to open up and talk to people about it, about what actually happened and I started hearing from more and more people like, yeah, that happened to me, too. Different branch of service, different decade, different whatever, but everything else was the same. (38-year-old woman, Air Force veteran: 7 years of service).

\section{Recommendation 7: Expand MST prevention training content for servicemembers and leaders}

Survivors identified six content areas that would make the training more relevant and engaging if implemented. First, there is a need for the subject matter to be more comprehensive so that it "gets to the root of the problem instead of reacting to the actions of somebody" (39-year-old woman, Air Force veteran: 22 years of service). Bystander intervention amongst peers needs to continue to be part of the message, which is consistent with the camaraderie and unit cohesion of the military culture. Survivors want to know that, as servicemembers and regardless of the situation, their peers and leaders will help defend them if attacked. One interviewee said, "The bystander culture of, you know, men not calling each other out I think is big. Addressing that in the training, you 
know, of bystander culture and some of that bullying stuff." (34-year-old woman, Army veteran: 10 years of service)

The second content area that needs improvement is reinforcing the message of what words and behaviors constitute acts of sexual harassment and sexual assault. Consequently, explicit and consistent clarification helps victims validate their experiences, seek the support they need, and perhaps feel empowered and safe to report the incident(s). A survivor recommended,

If I would have had the right tools and the right education, I would have known that it was actually rape. It would have been easier for me to probably come forward and say something and actually make a difference for other people. I'm positive that I probably wasn't the only person. (20-year-old woman, Army veteran: 3 years of service)

The third content area is finding ways to tie training content about MST into the larger context of military values and culture, specifically in the area of operational readiness. Survivors often talk about how service members, especially males, sitting in trainings either feel defensive about all men being characterized as rapists, or they feel disconnected to the subject matter, failing to see how sexual harassment and sexual assault applies to their specific unit or military job. Effectively communicating how the prevalence of MST adversely impacts the unit as a whole as a fighting force will help underscore the importance of preventing MST. An interviewee offered, In the military, our number one goal is readiness. You can't beat around the bush with this stuff. When we have a breakdown in our unit, like an assault or 
harassment that ruins our readiness, that perspective might be a good helpful tool for males especially 'cause sometimes I feel like males feel they're being attacked like, it's men's fault. I feel like they bring up gender too much. I feel like, if we could bring them more into "you need to care about these things because it's affecting our mission-readiness. We're supposed to be brothers and sisters in arms. We're supposed to take care of the people to your left and right. If you take this job really seriously and you want your unit to be successful, if you see something going wrong, why aren't you taking care of your battle buddy?" Because I feel like when, men feel they're being attacked, they go on the defensive and they shut down. [They are] not hearing the message. (24-year-old woman, Active Duty Army: 3 years of service)

Survivors also recommended expanding training content to include teaching boundary setting to service members. Doing so would have two benefits. First, prevention training should validate servicemembers' instincts for comfort, safety and survival. This is consistent with the situational awareness servicemembers and leaders are taught in the profession of arms, as noted by a participant:

Just because they're our company mates doesn't mean that they're more trustworthy or less trustworthy than anyone else. It's really, I think, listening to our instincts about the vibes we get from people, and, if we're not comfortable with somebody, then we should be encouraged not to be around them. (35-yearold woman, Army veteran: 4 years of service 
Teaching boundary setting also helps servicemembers feel empowered to directly address language or behavior that makes them uncomfortable as well as give them alternative options to address the situation via an intermediary such as another peer, $\mathrm{NCO}$ or Officer. A participant recommended,

Talk to them first. Let them know, “That's not right. I don't appreciate that. Please stop the behavior." If you don't feel comfortable speaking to somebody and telling them to knock it off, have a senior leader with you to speak with that person. At least talk to that person first before ruining that person's career over something that might've been as simple as "I was trying to get your attention. I was calling your name. You weren't listening to me. I put my hand on your shoulder to let you know I was behind you." That way you have somebody to say, "Look, I didn't feel comfortable." So that person knows, “okay. I'm sorry about that. Next time, I'll try to find a better way if you don't hear me to get your attention." Let somebody know that it bothers you. (40-year-old woman, Active Duty Army: 16 years of service)

Survivors also suggested explicitly including domestic violence in the training curricula. Oftentimes, the only people briefed on the prevalence of domestic violence are the unit leaders, and, as such, rank and file military members are unaware of the support and resources (legal, medical, social work, etc.) they can access either for themselves and/or their fellow service members. An interviewee recounted, I mean, as a leader in the Air Force, you sometimes hear about things that are going on in your squadron, but you don't hear about domestic violence or issues 
that are happening. The higher ups, do. They sit in a room, and they talk about all the issues that are happening around their base. So, a lot of times at the lower levels, you don't hear about those things unless it's in like the Base Blotter ${ }^{46}$ like months later or something like that. I don't even know if that would help, but I think reminding people what's happening around them is important. I think this stuff is happening, we need to be aware of it, don't just pretend and glaze over it. I would want my airmen to know that we are not immune to this. (40-year-old woman, Active Duty Air Force: 23 years of service)

The additional benefit of including a unit on domestic violence in the prevention training is that it helps decrease the isolation of domestic violence victims while raising the awareness of the role domestic violence plays in the prevalence of sexual harassment and sexual assault. This is especially relevant because of increasing numbers of dual military couples that currently serve.

Survivors recommended a fifth area in their training content: victim stories of healing and growth. One recommendation was to invite survivors to speak:

Maybe if survivors could address the groups telling their story about what happened, or just having a panel of them go and speak about their story to the groups, maybe that would be more effective than the typical military training is. A lot more powerful than having your sergeant major stand up there and giving a

46 The base blotter is the section of the military installation newspaper that lists recent criminal activity on post/base/station. 
briefing about behaving yourselves. (35-year-old woman, Army veteran: 4 years of service)

Although necessary information, training usually focuses on the logistics of reporting the incident(s) and the available resources. Consequently, servicemembers who are contemplating coming forward to report incidents are unable to imagine how they will survive the ordeal and what they can expect for their personal and professional lives.

The last training content recommendation concerned being more intentional about including narratives about male victims to counteract the mostly female victim-male perpetrator paradigm that seems to dominate the prevention training. Although all 21 of the survivors identified as women when they were sexually assaulted, there was an acknowledgement that male survivors tend to be an afterthought when talking about MST. One participant stated,

There are just more men in the military. Numbers. Men are being assaulted, too, but they don't really like touch on it more than that. It's more of just to like, "Hey! Men get raped too!" kind of thing. And if there were scenarios more about power and not about the sexual aspect. And there's that scrawny private that he can just overpower, and they're both male. And, it's not about the sexual aspect. It's about the power [that] comes through the sexual assault. I feel like, when we get into more of the intervention, that's when we spin back more of "victim is female, perpetrator is male." And I feel like that's when our guys kind of turn off, and they don't listen anymore. Because they don't want to feel like they're being attacked. (24-year-old woman, Active Duty Army: 3 years of service) 
Sixth, survivors also spoke about the need for the training message being focused on the shared responsibility for prevention amongst women and men, consistent with the military culture that grounded in camaraderie and unit cohesion. A participant said, I think the training should be coed [because] it would help curb a lot of that locker room talk, or whatever it is, that, if men see or hear something that's not right, that they step up to the plate and they report it as well. It's not just a woman's responsibility to report it. It's anybody that knows of something like it, and it should focus [on] not just the regular jokes of "Keep it in your pants," but this is not acceptable behavior. You need to take it seriously. It isn't a fucking joke. It's your inability to act like a decent human being, and it needs to be framed like that. Stepping up in support of their fellow members because you need to have each other's backs. If we don't, we're not effective units out there because they may be destroying unit integrity with their actions. It messes with trust in that situation. (35-year-old woman, Army veteran: 4 years of service)

\section{Recommendation 8: Improve the quality of the SAPR staff /trainers/facilitators}

The survivors identified four ways to improve the quality of the military's Sexual Assault Prevention and Response program (SAPR) staff, trainers and facilitators. First, survivors shared that facilitators should be expected to have a breadth and depth of subject matter competence for a variety of situations. However, just as important was the humility to learn more about the experiences of the other servicemembers attending the class, and, if necessary, track down the information and resources to a question or concern they were unable to answer in the training. Doing so helps build confidence in 
the prevention and response initiatives that the trainers are promoting in the class as well as building trust with the servicemembers themselves. An interviewee offered, I would really hope that they would be, like that their training process to become trainers would be super in depth and would cover a lot of the different scenarios so that, any time any student is taking their training and has a question and they seem off the wall, that, even if they don't have an answer for it, that they would know where to go to find the answer and that they would be able to get back to that person within a day or two with an answer, with a solution. (38-year-old woman, Air Force veteran: 7 years of service)

Second, interviewees also suggested that facilitators be trained in body language in terms of verbal and non-verbal cues to help inform how they engage with the servicemembers in attendance, especially to identify discomfort or defensiveness. Doing so would help the facilitator gauge if they need to follow up with certain individuals during a break or after training. A participant stated, I think that it would be helpful if they have body language training, so that they can pick up on whether somebody is uncomfortable with it, and they can follow up later on, or pick up on those body cues that people are giving out, and possibly be one of the first line of defenses to be watching and be like, "Hey, this person was really uncomfortable. Did something happen? Has something happened?” Or, if somebody is really dismissive and everything, like they can follow up or whatever and see if something has happened and has it been reported, or they could actually become one of the first lines of investigation, because they can find 
out, "has something happened?" Or, if it's a guy, that his responses are not matching up or whatever, maybe he is a perpetrator and he's being really dismissive because he's like, "Yeah. Whatever, I've done it. It's not a big deal." Type thing. But I think, really, just being aware of the body language people are giving out. They could become one of the first lines of defense against sexual assault. (35-year-old woman, Army veteran: 4 years of service)

Third, there were several attributes that survivors valued in their facilitator and which directly affected their views on the quality of the program. To begin with, the tone they set with their training attendees, especially when engaging in challenging or sensitive subject matter, was identified as making a difference in how the information is received. They noted how crucial it was that the facilitators not project or compare their own experiences onto another servicemember, even if they've shared similar circumstances. One stated,

Don't place judgment. Keep your personal experiences to yourself, for the most part, because not everybody has the same resilience. They weren't raised the same. So, be compassionate, I guess. But I think that's the biggest part. Your resiliency is not the same as their resiliency, and your specific situation is how you think you would handle it and how you did handle it is not the same as someone else's. (37-year-old woman, Active Duty Army: 20 years of service) Additionally, facilitators play an active role on how they can make their training content more memorable, and, just as important, be taken seriously. Many survivors talk about "check the box" training that consisted mostly of training NCOs reading off of 
PowerPoint slides, and how interaction impacts the effectiveness and level of engagement. One participant said,

I always hate sitting through the classes. I would say that the change that they've made where it's interactive [and] they do the presentations, then they do discussions. I honestly think that those are so much better than just a PowerPoint and just clicking through them, and just going over the same material. I think the discussions are a huge thing. I would say continue to do those. (40-year-old woman, Active Duty Army: 16 years of service)

Fourth, having an actual MST survivor sharing their experiences of healing and personal growth can help victims feel less isolated about what to expect as well as provide training attendees a personal and unique vantage point that can help educate them on the trauma incurred by the survivor. One survivor said,

I had an idea. I don't know, but, maybe if survivors of it could address the groups. Telling their story about what happened, and why they didn't, or why they did, or just having a panel of them go and speak about their story to the groups. And maybe have it "this is how it affects." And maybe that would be more effective than the typical military training is. (35-year-old woman, Army veteran: 4 years of service)

Study participants recommended using MST survivors as facilitators and victim advocates because they are in a unique position to increase awareness and sensitivity to the issues, as well as connect the relevant of MST to the overall military mission, even if 
servicemembers attending the training are initially unable to connect the dots. A survivor mentioned,

I really think that people just don't get it. They don't see how they personally are affected by it. I had one person in the last year, raised their hand and they were like, “This isn't an issue in my unit, so I don't see why I have to be here," that was when I kind of lost it. [I] didn't go off on them, but that was when I was like, "You know what? Thank you for saying that. Let me show you how it affects you." I started connecting the scenarios [to] the people. "Look, you may not get assaulted, you may not have been the person that assaulted someone, but somebody at work was assaulted and now they're having trouble keeping up with the workload or they're out, so now they're even more short-manned. It's gotta be realistic for people. You can't just read words off of a screen. You have to have some kind of connection or relativity to it for the people that you're, you know, presenting it to. It was just giving a space for people to share. And sharing a little bit of my experiences, you know, because people tend to listen when you, yourself, care about it. (39-year-old woman, Air Force veteran: 22 years of service)

In addition to using survivors to serve as facilitators, it was suggested to use other relevant staff members that support the SARC program, like physicians, mental health counselors, victim advocates and JAG officers to supplement the training content. By sharing their role in the overall process, they can help educate survivors about the options and resources available to them as well as on how to best support their fellow 
servicemember. It also helps increase familiarity and trust if servicemembers know exactly who is involved in the process. An interviewee wondered, I would also question if it was possible for a counselor or a physician, whatever the clinics are that each unit is assigned to, having them come to a training to give scenarios, examples of different ways that soldiers found ways to report safely (safe, legal way to do that). Have the people be there that have been reported to, and give examples of how they approached it with the actual people, the power of just human energy, and how a person experiencing the rapport of another person could make the difference about being comfortable with doing that or not. Because it takes out the unknown. Like having what's it called. (44-year-old woman, Army veteran: 4 years of service)

\section{Summary of Findings}

The participants in this study provided a window into their experiences surviving sexual harassment and sexual assault during their military service from 2003 to 2019 . Their experiences as survivors included how they were treated by the leadership and chain of command as well as their military unit as a whole in the aftermath of their MST. Survivors also provided insight into the quality of the messaging and follow through action of the military's prevention and response efforts towards incidents of sexual harassment and sexual assault, and how these efforts were (mis)aligned to the military's stated policies.

Answering the three research questions posed at the beginning of this study exists within the intersection of the four finding areas of culture, leadership, prevention and 
response that emerged from the interviews that contributed to the MST survivors' experience, as summarized in Figure 3. In addition to pointing out the multiple deficiencies in the military's prevention and response efforts, the survivors also identified what "right" looked like when stated policies matched their experiences of how they were treated by their chain of command and the leadership.

\section{Culture}

In the first theme, the culture of sexism and misogyny played a significant role in their day-to-day experiences as servicemembers and as current veterans. Whether their military service was minimized or devalued, or they were subjected to additional restrictions on their movement and social relationships, the common thread throughout this theme was the differing standards expected of women servicemembers versus their male counterparts. These differing standards manifested in the normalization of acts of sexual harassment and sexual violence as well victims being bullied and slut shamed by their perpetrators, peers, chain of command, and various leaders in the medical and Veterans Health Administration community.

\section{Leadership}

The second theme of leadership and chain of command matters focused on the various ways leaders influenced the command climate of their military unit. In multiple examples, survivors shared how their NCOs and officers played a direct role in modeling or excusing acts of sexual harassment and sexual assault, both stateside and overseas, even during wartime deployments. The majority of the participants shared incidents where the leaders in their chain of command either perpetrated the incidents themselves, 
made excuses for the perpetrators, or minimized the MST experiences when the survivors came forward to report the incidents. However, there were also examples where leaders in the survivors' chain of command played a significant role in their healing and recovery, facilitating access to the support and resources while reminding them of their strength, resiliency and overall value to their military unit.

\section{Response}

In the third theme, survivors shared how the military's response to their sexual assault was often (re) traumatizing due to how they were treated by their peers and the leaders in their unit, which sometimes resulted in professional and/or social retaliation. Consequently, the trauma manifested in feelings of isolation, resulting in significant mental health issues, substance abuse and self-destructive behaviors like eating disorders, cutting, and suicide attempts. Survivors were often hesitant to seek help or counseling because of the potential damage to their career and access to future veteran benefits. In some instances, the same medical or mental health professionals in the military and the VA hospital whom they were told they could reach out to for support and treatment participated in victim-blaming and minimized or erased their experiences. Additionally, the LGTBQ survivors who were sexually assaulted during the "Don't Ask, Don't Tell" reported feeling the additional layer of stress their career being threatened by the prospect of their sexual orientation being weaponized against them, being outed and possibly discharged from the military career. 


\section{Prevention}

The fourth theme focused on survivors experiencing the military's prevention efforts, focused on training, being inadequate. Survivors shared that the quality of the training was compromised by how the messaging and focus of the training was so far removed from the reality of the survivor's experience, especially with their military unit and chain of command. Another concern was the skillset of the prevention trainers and facilitators who were often unable to engage with sensitive subject matter such as consent and challenging rape myths. In contrast, the most effective prevention efforts involved trainers and leaders who were attentive and attuned to servicemembers' overall affect either inside or outside of training, and/or customized the training content for direct relevance to the training participants.

Last, the explicit recommendations the survivors shared during the interviews revealed a multitude of suggestions to improve the military's prevention and response efforts, which intersected the four theme areas. The spectrum of the recommendations ranged from leaders paying greater attention to modeling and enforcing professionalism, to increasing the consistency of accountability, to treating MST as seriously as other service-related injury and improving the survivors' access to resources and time to heal properly. The common thread that ran through the participants' recommendations was the disconnect between the military culture and stated policies which place a premium on values such as camaraderie, accountability, and discipline, and the MST survivors' experiences with how they were treated by their chain of command and leadership, as 
well as how acts of sexual harassment and sexual assault and victim-blaming were normalized. 


\section{Chapter Five: Discussion}

The goal of this qualitative study was to identify the roles that the military's messaging and gender identity played in both the MST survivor's experience, as well as the military's prevention and response efforts to MST. In addition, to sharing their experiences with sexual harassment and sexual assault during their military service, the survivors shared their recommendations on how the military's messaging, and its prevention and response efforts could be more survivor-informed. Centering the MST survivors as the key stakeholders helped shift the paradigm of subject matter expertise and problem solving from the conventional hierarchical decision-makers (like the military) to those with the actual lived experiences before, during, and after the incidents of sexual harassment and sexual assault.

The purpose of this discussion section is to identify the key takeaways from the findings as well as discuss how the findings move the literature forward. Additionally, the study's limitations, its implications and recommended areas for future MST research are discussed.

\section{Key Takeaways from the Study Findings}

There were four key takeaways that emerged from the study findings and the survivors' recommendations. The takeaways reinforced the perception that the Department of Defense treats the continued prevalence of sexual harassment and sexual assault as a peripheral and low priority issue impacting their servicemembers, and the U.S. military as a whole. 
The first takeaway is the simple acknowledgement that, despite some reforms to their MST prevention and response programs and victim support, the military has failed to effectively address incidents of MST. This is not due to a lack of awareness of the historical and current problems MST poses to the military in terms of mission readiness, recruitment, and retention. Rather, the failure comes from the military continuing to operate from within an anachronistic or outdated paradigm for its prevention and response strategy. One limit of the old paradigm is that women still do not have full parity with their male counterparts and are, thus, still viewed as "less" than men in the military, despite advances in women's inclusion in more military roles and occupational specialties. Another limit is the military's “one size fits all” approach to problem solving and strategy development, which seems to be based on the standard of white, cisgender heterosexual men. This approach is problematic because it fails to account for the overlapping power dynamics of identities historically minimized, erased or disenfranchised, which contradicts what is known about intersecting identities (Alford, 2016; Belkin, 2012; Crenshaw, 1991). The common thread that ran through what the participants shared about the MST survivor experience is that a paradigm needs to be stretched to discover new and more effective ways to prevent and respond to MST.

The second takeaway validates the significant roles that leaders play in the eradication of military sexual trauma within every branch of service. Both the positive and negative leadership examples that the survivors shared underscored that the success or failure of any MST prevention effort relies on its leaders setting the tone of what is expected of their subordinates in terms of professional standards, behavior, and 
accountability. Consequently, these leaders also have the power to dictate the culture as well as the quality of the military's prevention and response efforts. In short, military leaders are responsible for what their unit does or fails to do. Any meaningful and sustainable change to prevention and response efforts depend on leaders committing to making MST prevention an operational readiness priority.

Third, the findings also suggested that moving forward, the military's MST prevention and response strategy should ensure the quality of both the message (training content) and the messenger (trainers/facilitators and military leaders), for maximum effect. This strategy needs to be grounded in, and consistent with, the same military standards and values (ex. camaraderie, discipline, accountability, professionalism) that guide and govern servicemembers in their day to day lives. Additionally, the prevention strategy should have an equally robust response, where accountability and military justice (regardless of gender, rank or position) is consistently applied, and backed up by tangible action, where perpetrators are brought to justice. Furthermore, military leaders should avoid using absolutist terms likes "zero tolerance" for sexual harassment and sexual assault, unless they are prepared to follow it up with concrete action steps. Having an MST prevention and response strategy that is congruent with the military's justice system will be key in increasing trust and credibility within the military community, especially for the survivors.

Fourth, a crucial component of prevention programs would be having professionals who have a robust facilitator and adult education skill set that allows them to respond to difficult or sensitive subject matter including, but not limited to, questions 
(or joking) about consent, rape myth perpetration and bystander intervention. The ideal facilitators would be able to strike the delicate balance of providing necessary information about available support and resources, while simultaneously challenging service members who make inappropriate jokes about rape myths or issues about consent, all the while being sensitive to the possibility of having survivors of MST or other forms of physical/sexual abuse in the audience.

The above takeaways help support the following outcomes that are paramount to sustainable and meaningful change in the military regarding the MST prevention and response program. The common thread that runs through the three takeaways is that the quality of the message is just as important as the messenger in place to communicate it.

- demonstrate the relevance to MST prevention to every service member, regardless of gender, rank or position

- explicitly demonstrate ways that the mission and the members of the military unit can be both positively and negatively impacted if they fail to respond appropriately

- ensure that the MST prevention and response strategy aligns with the same values the military claims to espouse and be governed by (ex. camaraderie, discipline, accountability).

\section{How This Study Moves the Literature Forward}

The findings from this study align with and move the existing sexual violence literature forward within three discipline areas, the Department of Defense (DoD), as well as gender and communication studies. 
First, within the Department of Defense literature, the details of the military's prevention and response efforts were available via a variety of sources such as command climate surveys, focus groups and survivor testimonies before Congress (CSPAN 2013, 2014; DMDC, 2014, 2016, RAND National Defense Research Institute, 2014). The findings from this study help augment the DoD's prevalence data on sexual harassment and sexual assault (DoD, 2018; RAND National Defense Research Institute, 2014; United States Department of Veteran Affairs, 2016 by highlighting the strengths and weaknesses of their current prevention and response strategy. A lot of the study findings are consistent with what is known in the DoD such as the concern for professional retaliation for reporting, a lack of accountability for perpetrators and the perceived quality and effectiveness of the prevention training (DMDC, 2014, 2016; DoD, 2014; RAND National Defense Research Institute, 2014; Namrow, Hurley, Cook, Van Winkle, \& Hurley, 2016; Namrow, Hurley, Van Winkle, \& De Silva, 2016). However, my study advances the DoD-related literature by using the rich data from survivors to specifically focus on the messaging and what survivors are taking away from the training, specifically in the following areas:

- Centering and Empowering MST survivors as part of the improvement process in prevention and response efforts.

- Increasing the attention, focus and priority on the messaging and what survivors are taking away from the training 
- Underscoring the need to invest in a more robust evaluation process to assess the effectiveness of the military's prevention and response programs

The survivors shared the multiple ways that their actual experiences with MST and how they were treated by their chain of command and military unit were incongruent with the stated values of the military culture and intended policies of the military that place a premium on camaraderie, trust, accountability and discipline.

The findings in this study reflect the DoD literature (CSPAN 2013, 2014; RAND National Defense Research Institute, 2014), which shows the social and professional retaliation that survivors experience when they come forward to report the MST incidents. These retaliatory actions add to the perception that a higher penalty exists for victims who come forward to report the crimes, than the actual perpetrators committing the crimes (CSPAN 2013, 2014). Additionally, this study revealed that military leaders could prove to serve either as protective or risk factors in the promotion of a culture of sexism and misogyny, which is shared in the focus groups and congressional testimonies (CSPAN 2013, 2014; DMDC, 2014, 2016). This is contingent on the examples leaders set for their subordinates: whether they take seriously the threat of MST to "good order and discipline," whether they prioritize MST prevention training, and the manner that they choose to respond to a servicemember coming forward to report an incident (regardless of the rank or position of the perpetrator). Survivors in this study cited the time and resources extended to them by their leaders was a key component to their ability 
to heal both physically and mentally, as well as a factor in the military being able to retain them.

The study findings also validated the need for the military to invest more resources (time, money, personnel) into messaging, specifically how they frame the issues surrounding sexual assault. As subject matter experts in the field of communications and media assert (Mejia, Somji, Nixon, Dorman \& Quintero, 2015; Scheufele \& Tewskbury, 2007) how an issue is communicated or framed is just as important as the content of what is being said, especially when discussing sensitive subjects like sexual violence (Mejia et al., 2015). The status quo relies on presenting a "portrait" context of the etiology of sexual violence, which relies on the individual person (usually the victim) or the incident means reverting back to the status quo of holding the victims solely responsible, or pointing out that their inability or failure to prevent their victimization was a result of their weakness or shortcomings. Additionally, by including both elements of sexual harassment and sexual assault in the study, instead of solely focusing on sexual assault, it helps underscore the need to present MST within a more wholistic spectrum of violence, which is congruent with the National Sexual Violence Center and the DoD's acknowledgement that MSH and MSA lies on a "continuum of harm" (DoD, 2014a, p.21).

A second component of the sexual violence prevention literature comes from the discipline of gender studies, specifically the concepts of RMA, gender role traditionality and ambivalent sexism (Carroll, 2016; Glick \& Fiske, 1997; Payne, Lonsway \& Fitzgerald, 1999) which are a significant part of my study. The dual components of 
ambivalent sexism (benevolent and hostile sexism) are reflected in the findings concerning military culture and the strategy of military leaders used strategies like gender-specific curfews, and restricting women's movements as ways to prevent sexual harassment and sexual assault. The participants who rejected the benevolent sexist norms by abusing alcohol and socializing with whom they wanted to were punished by their leaders, and they became solely responsible for their own victimization, more so than the actual perpetrator. Survivors also shared that the acts of attempted or completed sexual violence they experienced (a form of hostile sexism) were compounded by social and professional retaliation they experienced from their peers, as well as their leaders in their military unit, especially if they were rejecting the perpetrators advances.

The RMA and ambivalent sexism literature provided the language in my study not only to name the problematic victim-blaming behavior that many of the survivors experienced from their military leaders, but it did highlight the importance of organizations like the military being more intentional and conscious of how their MST prevention and response efforts either promote or challenge various types of sexism and rape myths. For example, part of my study's findings was congruent with Yamawaki's 2007 study exploring the roles that benevolent sexism, hostile sexism and gender role traditionality played into the degree that participants would blame the victim and/or excuse the perpetrator's behavior. Yamawaki's findings that benevolent sexism and gender role traditionality were significant predictors of victim-blaming for date rape scenarios and that the women who adhered to strict gender role traditionality would most likely benefit from protected and deferential status. Whereas their female peers who 
didn't obey socially acceptable norms would tend to be less protected, and most likely be blamed for their own sexual victimization.

Additionally, Carroll's (2016) study that surveyed male and female students in both the civilian university and military service academies underscored an important point that the survivors in our study shared their recommendations in how to improve MST prevention and response programs. That is that any initiatives must be intentionally informed by the unique organizational culture and workplace settings, versus applying aggregated scores from the ambivalent sexism inventory and RMA scales. Doing so would mean developing more intra-scale analyses to explore the nuance of attitudes towards victims and perpetrators so as to be able to identify additional confounding variables and predictors for effectively addressing sexual violence within the military.

Third, the field of communication studies and its applicable literature of messaging within sexual violence literature provided a lens to review the survivors' perceptions of the military's prevention and response efforts in my study. For example, in Mejia et al.'s (2015) content analysis examining the media's coverage of sexual violence, they found that sexual violence is portrayed within a myopic criminal justice frame, where survivors and sexual prevention advocates were given limited to no roles as subject matter experts, which is congruent to my study's findings where survivors experiences do not feel represented in the military's MST prevention programming and response efforts, especially in their training components. Additionally, O’Neil and Morgan's social discourse analysis (2010) highlighted the importance of the training content reflecting the continuum of behaviors that is sexual violence (to include 
attempted as well as completed acts), that engages participants beyond the good/bad binary of predatory behavior. It also underscored the need for a more comprehensive view that draws attention to issues about consent, harm and victimization, which is consistent with what the survivors in my study were recommending on how to improve the quality and content of the training. Where my study moves the communications discipline forward is in intentionally moving survivor centered MST studies like mine into the prevention space using messaging as the vehicle for change and advocacy.

\section{Limitations of the Study}

There were two main limitations to this study which should be considered when considering the findings. A first limitation is the remote recollections of the participants MST experience, especially their memory about the type and quality of the MST prevention training they sat through and their chain of command/military unit's response, which, depending on when they served in the last 15 years, was challenging for some of them to recall in detail. Many of the survivors had difficulty remembering the type and quality of the MST prevention training they sat through, and their chain of command/military unit's response. Additionally, a few of the survivors talked about past experiences where military policies have since changed or have been reformed, as exemplified by the repeal of Don't Ask, Don't Tell in 2010, and the option for victims to request an expedited transfer to another unit so as to avoid their perpetrator/abuser.

The second limitation is the overall demographic representation of the participants, as highlighted by Table 1 in Chapter 4. A majority (81\%) of survivors in this study identified as non-hispanic/latino(a) white, and all the participants were women, 
with the exception of one transgender man. Despite my best efforts, I was unable to obtain increased diversity within the gender, racial, ethnic, and sexual orientation identities I was aiming for in my sample. However, it was helpful to reexamine my eightmonth participant outreach and recruitment strategy and identify the lessons learned for conducting future MST-related studies, that would benefit not just myself but other researchers.

When I started recruiting participants in October 2018, my participant outreach to meet my 20-25 participant goal consisted primarily of sharing my one-page flyer with my extensive personal and professional network within the military/veteran communities, as well as in the civilian research and advocacy communities, via email. I also solicited participants via my personal Facebook page and other closed Facebook member groups, including Latinas Completing Doctoral Degrees, Army Women Officers, Minority Veterans of America, and Transgender Veterans of America.

As I struggled to recruit participants who met the criteria, I consulted with other veteran advocates for additional ideas on how to widen my search and improve my outreach efforts, which I implemented. Their suggestions included, but were not limited to, reaching out to sexual violence prevention organizations and veteran advocacy groups like the Center for Victim Research. The suggestion that yielded the most participants was the creation of a dedicated Facebook page for the study, which not only spread awareness of the study, but provided a safe and no/low pressure way for an MST survivor to learn more about the study and consider participating. Additionally, I interviewed seven key informants/stakeholders who included male MST survivors, a transgender 
veteran advocate, and government civilians who work in the military's family services and SAPR programs to gain more insight into the quality of the military's MST prevention and response efforts as well as into ways to improve outreach for MST research participants.

Reflecting on my recruitment strategy, there were three key lessons learned that I would use for future MST studies as well as recommend to other researchers. First, I would have created an advisory group (made up the same diversity of key informants that I interviewed towards the end of my study) as a resource to confer with before I submitted the IRB application, as well as throughout the study, especially during the participant recruitment phase. Second, I would have included a more robust and comprehensive recruiting and consulting strategy in my IRB application, to include the possibility of using a dedicated Facebook page (or other social media platform) to recruit study participants as well as use an advisory group as a resource during the recruitment and data collection phase. Doing so would have saved me considerable time and momentum in having to submit multiple IRB amendments and wait for approval. Third, I would have had prepared my Facebook page (or alternative social media platform) earlier in the recruitment process. Employing this three- prong outreach and recruiting strategy would have enabled me to reach more potential study participants, while drawing from the expertise of an advisory group when I hit roadblocks for participant enrollment and/or sample diversity. 


\section{Implications}

Building off the insightful suggestions proposed by the survivors to improve the prevention and response efforts, and the best practices gleaned from the empirical literature reviewed for this study, our study has several implications for three key community stakeholder groups.

\section{Military and Veteran Communities}

Our findings suggest that the military and the veteran communities should address four areas in order to strengthen their MST prevention and response efforts, and support survivors more effectively. First, the military could support survivors more effectively if they initiate, and develop a more intentional communication strategy for MST prevention and response that is reflective of a modern $21^{\text {st }}$ century workforce and Department of Defense. Second, the military should invest in a more formal evaluation of MST prevention and response programs and efforts, collaborating with, and integrating the "best practices" of sexual violence prevention education and communication strategies from the civilian community. Third, the military needs to expand the content of the MST prevention program so as to include, but not limited to, components on consent, boundary setting, and domestic violence. Fourth, the military and veteran communities should commit to a continuous investment in MST research that includes and empowers survivors in more intentionally participatory roles would benefit their overall programming so as to become more survivor informed and authentically reflective of the survivors' experiences with MST. 


\section{Non-Military Communities}

This study also has implications for non-military communities. Our findings suggest that the fields of sexual violence prevention and victim advocacy would benefit from continuing to invest in sexual violence prevention education at both the K-12 and college/university levels. The non-military community would also be best served if they promoted survivor-centered sexual prevention research. This community would also benefit from being intentional about including survivors, not just as study participants, but throughout the research process, to include, but not limited to, roles such as subject matter experts, program evaluators and study researchers.

\section{Field of Social Work Education}

There are also some important implications for the field of social work in two key areas. First, this study reminds us of the importance of integrating macro social work component into the curriculum for a more comprehensive understanding of MST and opportunities for advocacy, such as policy. Second, the field of social work would benefit from expanding “insider/outsider” research (Acker, 2000) in social work programs, which in turn would help build the capacity of military veterans as social workers.

\section{Future Research}

Finally, these findings highlight three areas of opportunity for future MST research in multiple areas. First, it would be helpful if the military identify and (re)define what meaningful outcomes look like for MST prevention programs, so as to move beyond using MST reporting rates as success or failure, depending on whether it rises or falls. 
Second, the military would greatly benefit from developing a formal evaluation of MST prevention programs with survivors as co-stakeholders and investigators. Doing so would help create a baseline of effectiveness, measure the health and efficacy of the prevention programs, and identify the strengths and deficiencies. Employing patient centered outcome research methodology would directly elevate survivor voices and allow them to play a more direct role in the MST prevention and response efforts. This in turn, would mark a significant shift in subject matter expertise and increase the quality of the programming so that they are more reflective of the survivor experiences.

Third, future MST survivor-based research should be more intentional about including more survivors from communities of color, cisgender men and transgender servicemembers, all which tend to be underrepresented in MST research. It is crucial to ensure that these prevention programs reflect the survivor voice of the LGBTQ community and include the full spectrum of gender identity and sexual orientation. Doing so as would help challenge the norms around gender-based stereotypes surrounding the binary female victim-male perpetrator paradigm that dominates the risk reduction and bystander intervention elements of MST prevention programming.

\section{Conclusion}

Servicemembers from the Army, Navy, Air Force, Marine Corps and the Coast Guard continue to be subjected to sexual harassment and sexual assault, despite the military's proclamation of a zero-tolerance environment and multiple reforms made to their MST prevention and response strategy. The military's strategy has relied primarily on MST prevention training and its leaders to communicate expectations and formal 
policies regarding sexual harassment and sexual assault. Regardless of some of the improvements to the prevention and response protocols, survivors of MST have noted the disconnect between their experiences, the military's stated policies, and, especially, its organization culture of camaraderie, accountability, and discipline.

This gap between the MST survivors' experience and the military's prevention and response efforts became the focus of my dissertation and this qualitative study. The objectives of the study were to identify how the current messaging in the military affects the experiences of MST survivors and to obtain recommendations on how to improve the military's prevention and response efforts.

This qualitative study, based on interviews with 21 survivors who experienced sexual harassment and sexual assault during their military service from 2003 to 2019, had four key findings. The intersection of the MST survivor experience lies at (a) the military's culture of sexism and misogyny, (b) the leadership and the command climate (c) response efforts that are often (re)traumatizing, and (d) often inadequate prevention efforts, particularly in regard to training.

The MST survivors' experience, including their recommendations for its improvement, underscored the multiple gaps in the military's prevention and response strategy. This study supplements the existing empirical literature about prevention and intervention strategies, communicating sexual violence incidents, and the DoD's prevalence data on sexual harassment and sexual assault. In addition to highlighting the military's deficiencies, the survivors' experiences also pointed out examples of what a positive survivor-centered prevention and response program looked like. 
The study findings also highlighted the importance for the military to be more intentional about aligning the messaging regarding MST prevention and response policies, the values of the military culture, and the survivors' experience. The study findings also validated the crucial role that leaders play in modeling the military values and culture and supporting survivor-centered prevention and response efforts.

Additionally, this study showcased the need for the military to formally evaluate its prevention and response strategy to identify the strengths and weaknesses and be intentional in addressing them when apply reforms to the DoD's overall SAPR Program.

This study lays the foundation for future research and has implications for the military and veteran communities, non-military communities, and the field of social work education and practice. MST prevention and response strategy should intentionally focus on leveraging the diversity of the military, drawing from underrepresented groups from communities of color, and across the gender identity and sexual orientation continuum. Future research should challenge a one-size-fits-all approach to sexual assault prevention and response, and should be intersectional in approach, addressing the power dynamics that impact the identities of marginalized groups. 


\section{References}

Abrams, K. (1993). Gender in the military: Androcentrism and institutional reform. Law \& Contemporary Problems, 56, 217.

Abrams, D., Viki, G. T., Masser, B., \& Bohner, G. (2003). Perceptions of Stranger and Acquaintance Rape: The Role of Benevolent and Hostile Sexism in Victim Blame and Rape Proclivity. Journal of Personality and Social Psychology, 84(1), 111125. https://doi.org/10.1037/0022-3514.84.1.111

Acker, S. (2000). In/out/side: Positioning the researcher in feminist qualitative research. Resources for Feminist Research, 28.

Ackerly, B., \& True, J. (2010). Framework for Research Ethics and Evaluation: Justification and Guidelines. In Doing Feminist Research in Political and Social Science. Basingstoke; New York: Palgrave Macmillan.

Alford, B., \& Lee, S. J. (2016). Toward complete inclusion: Lesbian, gay, bisexual and transgender military service members after repeal of don't ask, don't tell. Social Work, 61, 257-265. doi:10.1093/sw/sww033

Allsep, L. M. (2013). The Myth of the Warrior: Martial Masculinity and the End of Don't Ask, Don't Tell. Journal of Homosexuality, 60, 381-400.

https://doi.org/10.1080/00918369.2013.744928

Baldor, L. C. (2018, April 26). Pentagon chief calls sexual assault a cancer in military. The Military Times. Retrieved on May 7, 2018 from https://www.militarytimes.com/news/your-military/2018/04/26/pentagon-chiefcalls-sexual-assault-a-cancer-in-military/ 
Banyard V. (2008). Measurement and correlates of prosocial bystander behavior: the case of interpersonal violence. Violence Victims, 23: 85-99.

Becker-Blease, K.A., \& Freyd, J.J. (2006). Research participants telling the truth about their lives: The ethics of asking and not asking about abuse, American Psychologist, 61, 218-226.

Belkin, A. (2012). Bring Me Men: Military Masculinity and the Benign Facade of American Empire, 1898-2001. New York, N.Y.: Columbia University Press.

Bell, K., Stein, S., \& Hurley, R. (2017). When Public Institutions Betray Women: News Coverage of Military Sexual Violence Against Women 1991-2013. Journal of Interdisciplinary Feminist Thought, 10(1).

Belkin, A. (2008). Spam Filter: Gay Rights and the Normalization of Male-Male Rape in the U.S. Military. Radical History Review.

Bem, S. L. (1993). The lenses of gender: Transforming the debate on sexual inequality. Yale University Press.

Berkeley Media Studies Group (2018a). Worksheet: Message Development. Retrieved on April 2, 2018 from http://www.bmsg.org/sites/default/files/bmsg_message_development_worksheet. pdf

Berkeley Media Studies Group (2018b). Resources: Framing 101. Retrieved on April 2, 2018 from http://www.bmsg.org/resources/framing-101 
Bourke, B. (2014). Positionality: Reflecting on the Research Process. The Qualitative Report How, 19(33), 1-9. Retrieved from http://nsuworks.nova.edu/tqr/vol19/iss33/3/

Brangham, W. \& Woodruff, J. (2018). After explicit photo scandal, Marine Corps grapples with culture of disrespect for women. U.S.A.: PBS News Hour. Retrieved from https://www.pbs.org/newshour/show/after-explicit-photo-scandalmarine-corps-grapples-with-culture-of-disrespect-for-women

Braun, V. \& Clark, V. (2006). Using thematic analysis in psychology. Qualitative research in psychology. 3:77-101. doi:10.1191/1478088706qp063oa.

Breiding, M.J., Chen J., \& Black, M.C. (2014). Intimate Partner Violence in the United States - 2010. Atlanta, GA: National Center for Injury Prevention and Control, Centers for Disease Control and Prevention.

Bronfenbrenner U. (1977). Toward an experimental ecology of human development. American Psychology, 32(7): 513-531.

Burn S. (2009). A situational model of sexual assault prevention through bystander intervention. Sex Roles. 60: 779-792.

Burt, M. R. (1980). Cultural myths and supports for rape. Journal of Personality and Social Psychology, 38, 217-230.

Butler, J. (1988). Performative Acts and Gender Constitution: An Essay in Phenomenology and Feminist Theory. Theatre Journal, 40(4), 519-531. Retrieved from http://links.jstor.org/sici?sici=01922882(198812)40:4<519:PAAGCA>2.0.CO;2- C 
Caplan, P.J. (Spring 2013) Sexual Trauma in the Military: Needed Changes in Policies and Procedures. Women's Policy Journal of Harvard. 10-21.

Cantor, D., Fisher, B., Chibnall, S., Townsend, R., Lee, H., Bruce, C., \& Thomas, G. (September 21, 2015). Association of American Universities (AAU), Report on the AAU Campus Climate Survey on Sexual Assault and Sexual Misconduct. Retrieved from https://www.aau.edu/uploadedFiles/AAU_Publications/AAU_Reports/Sexual_As saultCampus_Survey/Report\%20on\%20the\%20AAU\%20Campus\%20Climate $\% 2$ 0Survey\%20on\%20Sexual\%20Assault\%20and\%20Sexual\%20Misconduct_pdf

Carbado, D. W. (2013). Colorblind Intersectionality. Signs: Journal of Women in Culture and Society, 38(4), 811-845.

Carbado, D. W., Crenshaw, K. W., Mays, V. M., \& Tomlinson, B. (2013). Intersectionality: Mapping the Movements of a Theory. Du Bois Review: Social Science Research on Race, 10(2), 303-312. https://doi.org/10.1017/S1742058X13000349

Carroll, M. H., Rosenstein, J. E., Foubert, J. D., Clark, M. D., \& Korenman, L. M. (2016). Rape myth acceptance: A comparison of military service academy and civilian fraternity and sorority students. Military Psychology, 28(5), 306-317. https://doi.org/10.1037/mil0000113

Center for Disease Control and Prevention (CDC: March 25, 2015). The Public Health Approach to Violence Prevention. Retrieved on April 25, 2018 from https://www.cdc.gov/violenceprevention/overview/publichealthapproach.html 
Chapleau, K. M., Oswald, D. L., \& Russell, B. L. (2008). Male Rape Myths. Journal of Interpersonal Violence, 23(5), 600-615. https://doi.org/10.1177/0886260507313529

Charmaz, K. (2004). Grounded theory. In S. Hesse-Biber \& Levy (Eds.), Approaches to qualitative research: A reader on theory and practice (pp.496-521). New York: Oxford University Press.

Childress, S. (2013, May 10). Why the Military has a Sexual Assault Problem. PBS Frontline. Retrieved from http://www.pbs.org/wgbh/frontline/article/why-themilitary-has-a-rape-problem/

Cho, S., Crenshaw, K. W., \& McCall, L. (2013). Toward a Field of Intersectionality Studies: Theory, Applications, and Praxis. Signs: Journal of Women in Culture and Society. https://doi.org/10.1086/669608

Clift, E. (2013, May). Air Force General Blames Increase in Military Rape on Hookup Culture. The Daily Beast. Retrieved from http://www.thedailybeast.com/articles/2013/05/08/air-force-general-blamesincrease-in-military-rape-on-hookup-culture.html

Cohen, Z., \& Browne, R. (2018). US military sees spike in sexual assault reports. Cable News Network/CNN. Retrieved on May 7, 2018 from https://www.cnn.com/2018/04/30/politics/dod-sexual-assault-report2017/index.html

Coker, A. L., Fisher, B. S., Bush, H. M., Swan, S. C., Williams, C. M., Clear, E. R., \& DeGue, S. (2015). Evaluation of the Green Dot Bystander Intervention to Reduce 
Interpersonal Violence Among College Students Across Three Campuses. Violence Against Women, 21(12), 1507-1527. https://doi.org/10.1177/1077801214545284

Concannon, T., Meissner, W., Grunbaum, P., McElwee, J., Guise, A., Santa, N., Leslie, H. (2012). A New Taxonomy for Stakeholder Engagement in Patient-Centered Outcomes Research. Journal of General Internal Medicine, 27(8), 985-991. Corbin Dwyer, S., \& Buckle, J. L. (2009). The Space Between: On Being an InsiderOutsider in Qualitative Research. International Journal of Qualitative Methods, $8(1), 54-63$.

Crenshaw, K. (1991). Mapping the Margins: Intersectionality, Identity Politics, and Violence against Women of Color. Stanford Law Review, 43(6), 1241-1299. Creswell, J.W. \& Clark, V.L.P. (2011). Collecting data in mixed methods research. In J.W. Creswell \& V.L.P Clark (Eds.), Designing and conducting mixed methods research (pp.171-202). Thousand Oaks, CA: Sage Publications.

Cromer, L. D., \& Newman, E. (2011). Research Ethics in Victimization Studies: Widening the Lens. Violence Against Women, 17, 1536-1548. https://doi.org/10.1177/1077801211436365

C-SPAN (2013, March 13). Sexual Assault in the Military: Part I. Retrieved from https://www.c-span.org/video/?311468-1/sexual-assault-military-part-1 C-SPAN (2014, February 26). Military Sexual Assault. Retrieved from https://www.cspan.org/video/?318008-1/hearing-sexual-assaults-military 
CSPAN (2014). The relationship between military sexual assault, post-traumatic stress disorder and suicide, and on the Department of Defense and on the Department of Veteran Affairs Medical Treatment and management of victims of sexual trauma. Retrieved from https://www.c-span.org/video/?318008-1/hearing-sexual-assaultsmilitary

C-SPAN (2017, March 14). Marine Corps Social Media Nude Photos Investigation. Senate Armed Services Committee. Retrieved from https:/www.cspan.org/video/?425339-1/senators-press-marine-corps-commanderaccountability-nude-photo-controversy

Dahlberg LL, Krug EG (2002). Violence: a global public health problem. In: Krug E, Dahlberg LL, Mercy JA, Zwi AB, Lozano R, eds. World Report on Violence and Health. Geneva, Switzerland: World Health Organization; 1-56. Retrieved on April 25, 2018 from http://www.who.int/violence_injury_prevention/violence/world_report/en/chap1.p df

Defense Manpower Data Center /DMDC (2012) Human Resources Strategic Assessment Program. Workplace and Gender Relations Survey of Active Duty Members. Retrieved from http://www.sapr.mil/index.php/research Defense Manpower Data Center (DMDC). (2014). 2014 Department of Defense Report of Focus Groups on Sexual Assault Prevention and Response. Fort Belvoir, VA. Retrieved from 
http://www.sapr.mil/public/docs/reports/FY14_POTUS/FY14_DoD_Report_to_P OTUSAnnex_3_DMDC.pdf

Defense Manpower Data Center (DMDC). (2014). 2014 Department of Defense Report of Focus Groups on Sexual Assault Prevention and Response. Alexandria, VA. https://doi.org/DMDC 2014-041

Defense Manpower Data Center (DMDC). (2016). Annual Report on Sexual Harassment and Violence at the Military Service Academies (Academic Program Year 20142015). Washington D.C. Retrieved from http://sapr.mil/public/docs/reports/MSA/APY_14-15/APY_1415_MSA_Full_Report.pdf Department of Defense. (2014a). 2014-2016 DOD SAPR Strategic Plan. Washington D.C. Retrieved from http://sapr.mil/public/docs/reports/SecDef_Memo_and_DoD_SAPR_Prev ention_Strategy2014-2016.pdf

Department of Defense. (2014a). Fiscal Year 2013 Department of Defense Report of Substantiated Incidents of Sexual Harassment in the Armed Forces. Washington D.C. Retrieved from http://diversity.defense.gov/Portals/51/Documents/DoD FINAL REPORT-FY 13 Incidents of Sexual Harassment Report_15MAY2014.pdf Department of Defense. (2014b). Provisional Statistical Data on Sexual Assault Fiscal Year 2014: Report to the President of the United States on SAPR. Retrieved from 
http://sapr.mil/public/docs/reports/FY14_POTUS/FY14_DoD_Report_to_POTUS _Appendix_A.pdf

Department of Defense. (2014c). 2014-2016 DOD SAPR Strategic Plan. Washington

D.C. Retrieved from

http://sapr.mil/public/docs/reports/SecDef_Memo_and_DoD_SAPR_Prevention_ Strategy_2014-2016.pdf

Department of Defense (2015a). Sexual Assault Prevention and Response Strategic Plan.

Retrieved from

http://www.sapr.mil/public/docs/reports/SecDef_SAPR_Memo_Strategy_Atch_2 0150126.pdf

Department of Defense. (2015b). FY 2014 Department of Defense Fact Sheet

Washington D.C. Retrieved from

http://www.defense.gov/Portals/1/features/2015/0415_sexual-

assault/Fact_Sheet_FY1 DoD_SAPR_Annual_Report.pdf

Department of Defense. (2014b). Report to President of the United States on SAPR.

Washington D.C. Retrieved from

http://www.sapr.mil/public/docs/reports/FY14_POTUS/FY14_DoD_Report_to_P OTUS_SAPRO_Report.pdf

Department of Defense (2016a). Annual Report on Sexual Harassment and Violence at the Military Service Academies, Academic Program Year 2014-2015. Retrieved from http://sapr.mil/public/docs/reports/MSA/APY_14-15/APY_14-

15_MSA_Report.pdf 
Department of Defense (2017). Department of Defense Annual Report on Sexual Assault in the Military, Fiscal Year 2016. Retrieved from http://www.sapr.mil/public/docs/reports/FY16_Annual/FY16_SAPRO_Annual_R eport.pdf

Department of Defense (2018). Department of Defense Annual Report on Sexual Assault in the Military, Fiscal Year 2017. Retrieved from http://sapr.mil/public/docs/reports/FY17_Annual/DoD_FY17_Annual_Report_on _Sexual_Assault_in_the_Military.pdf

Department of Justice (2004). Office of Justice Programs, Bureau of Justice Statistics, American Indians and Crime, 1992-2002.

De Vogue, A. (2017, August 28). Transgender service members sue over Trump military ban. CNN Politics. Retrieved from http://www.cnn.com/2017/08/28/politics/transgender-service-members-militaryban/index.html

Dick, K. (2012). The Invisible War (Documentary). U.S.A.: Cinedigm/Docurama.

Entman, R. M. (2002). Framing: Towards clarification of a fractured paradigm. In D. McQuail (Ed.), McQuail's Reader in Mass Communication (pp. 391-397). London; Thousand Oaks, Calif.: Sage Publications Inc.

Frank, N. (2010). How Gay Soldiers Serve Openly Around the World. National Public Broadcasting (NPR). Retrieved from http://www.npr.org/2010/12/07/131857684/how-gay-soldiers-serve-openlyaround-the-world 
Garcia, S.E. (2017, October 20). The Woman Who Created \#MeToo Long Before Hashtags. The New York Times. Retrieved from https://www.nytimes.com/2017/10/20/us/me-too-movement-taranaburke.html?_r=0

Garcia-Rojas, C. (2012). Reporting on Rape and Sexual Violence. Chicago Taskforce on Violence Against Girls and Young Women: A Media Toolkit for Local and National Journalists to Better Media Coverage. Retrieved from http://www.chitaskforce.org/wp/wp-content/uploads/2012/10/Chicago-TaskforceMedia-Toolkit.pdf

Gitterman, A., \& Heller, N. (2011). Integrating Social Work Perspectives and Models with Concepts, Methods and Skills with Other Professions' Specialized Approaches. Clinical Social Work Journal, 39(2), 204-211.

Glaser, B.G., \& Strauss, A.L. (1967). The discovery of grounded theory: Strategies for qualitative research. Chicago: Aldine.

Glick, P., \& Fiske, S. (1996). The ambivalent sexism inventory: Differentiating hostile and benevolent sexism. Journal of Personality and Social Psychology, 70, 491512.

Glick, P.\& Fiske, S. (1997). Hostile and benevolent sexism: Measuring ambivalent sexist attitudes toward women. Psychology of Women Quarterly, 21, 119-136

Glick, P., \& Fiske, S. (1999). The Ambivalence toward Men Inventory: Differentiating hostile and benevolent beliefs about men. Psychology of Women Quarterly, 23, 519-536 
González-Prats, M.C. (2017): Accountability, Complacency, or Obfuscation? An Analysis of the U.S. Military's Prevention and Response Efforts to Military Sexual Trauma, Public Integrity. http://dx.doi.org/10.1080/10999922.2017.1278668

Gorislavsky, E. (2014). Racial-Ethnic Differences in Rape and Sexual Assault Victimization: A Pooled Analysis of NCVS Data, 1994-2010. University of Missouri-St. Louis.

Grbich, C. (2013). Qualitative data analysis: An introduction (2nd ed.). London; Thousand Oaks, Calif.: SAGE Publications.

Green, E.R. \& Maurer, L.M. (2015). The Teaching Transgender Toolkit: A Facilitator's Guide to Increasing Knowledge, Decreasing Prejudice and Building Skills. Ithaca, NY: Planned Parenthood of the Southern Finger Lakes: Out for Health. Retrieved from www.teachingtransgender.com

Grbich, C. (2013). Qualitative data analysis: An introduction. London; Thousand Oaks, Calif.: SAGE Publications.

Gurung, S., Ventuneac, A., Rendina, H. J., Savarese, E., Grove, C., \& Parsons, J. T. (2017). Prevalence of Military Sexual Trauma and Sexual Orientation Discrimination Among Lesbian, Gay, Bisexual, and Transgender Military Personnel: a Descriptive. Sexuality Research and Social Policy. https://doi.org/10.1007/s13178-017-0311-z

Gusfield, J. (1981). The culture of public problems: Drinking-driving and the symbolic order. Chicago: University of Chicago Press 
Halloran, L. (2013, May 25) Stunned by Military Sex Scandals, Advocates Demand Changes. NPR. Retrieved from http://www.npr.org/2013/05/23/186335999/stunned-by-military-sex-scandalsadvocatesdemandchanges? $\mathrm{ft}=1 \& \mathrm{f}=1122$

Hancock, A.-M. (2007). When Multiplication Doesn’t Equal Quick Addition: Examining Intersectionality as a Research Paradigm. Perspectives on Politics, 5(1), 63-79.

Hannagan, R. J. (2016). “I believe we are the fewer, the prouder.” Women's agency in meaning-making after military sexual assault. Journal of Contemporary Ethnography. Advance online publication. doi:10.1177/0891241616636664

Hanson, R., Morton, K., \& Harris, A. (2003). Sexual offender recidivism risk: What we know and what we need to know. Annals of the New York Academy of Sciences, 989, 154-66.

Harned, M. S., Ormerod, A. J., Palmieri, P. A., Collinsworth, L. L., \& Reed, M. (2002). Sexual Assault and Other Types of Sexual Harassment by Workplace Personnel: A Comparison of Antecedents and Consequences. Journal of Occupational Health Psychology, 7(2), 174-188.

Harris, G.L.A., Sumner, R.F., Gonzalez-Prats, M.C. (2018.). Women Veterans: Lifting the Veil of Invisibility. Taylor and Francis Group/Routledge Press, New York, NY.

Hart, N \& Crawford-Wright, A. (1999). Research as therapy, therapy as research: ethical dilemmas in new-paradigm research. British Journal of Guidance \& Counselling, 27(2), 205. 
Hertzog, Jodie, \& Yeilding, R. (2009). College Women's Rape Awareness and Use of Commonly Advocated Risk Reduction Strategies. College Student Journal, 43(1).

Herz, M. \& Johansson, T. (2015) The Normativity of the Concept of Heteronormativity, Journal of Homosexuality, 62:8, 1009-1020, DOI:

$10.1080 / 00918369.2015 .1021631$

Hesse-Biber, S., \& Leavy, Patricia. (2006). The practice of qualitative research. Thousand Oaks: SAGE Publications.

Hole, R. (2007). Working Between Languages and Cultures Issues of Representation, Voice, and Authority Intensified. Qualitative Inquiry, 13(5), 696-710.

Holstein, J.A. \& Miller, G. (1990). Rethinking Victimization: An Interactional Approach to Victimology. Symbolic Interaction, 12 (1). https://doi.org/10.1525/si.1990.13.1.103

Horgan, D. (Producer). (2013, May 7.) How the military fails miserably to address sexual assault. All in with Chris Hayes (TV Broadcast) New York, NY: MSNBC.

Hoyt, T., Klosterman Rielage, J., \& Williams, L. F. (2011). Military sexual trauma in men: A review of reported rates. Journal of Trauma \& Dissociation, 12(3), 244260.

Hulley, S. (2007). Designing clinical research (3rd ed.). Philadelphia, PA: Lippincott Williams \& Wilkins.

Human Rights Watch. (2015). Sexual assault and the LGBTQ community. Retrieved from http://www.hrc.org/resources/ sexual-assault-and-the-lgbt-community 
Hunnicutt, G. (2009). Varieties of Patriarchy and Violence Against Women: Resurrecting "Patriarchy" as a Theoretical Tool. Violence Against Women, 15(5), 553-573.

Hunter, M. (2007). Honor betrayed: Sexual abuse in America's military. Fort Lee, N.J.: Barricade Books.

Januta, A. (n.d.). Zero Tolerance: The Defense Department's Decades-Long Failure to End Sex Crimes in Uniform. The War Horse. Retrieved from https://www.thewarhorse.org/defense-department-failed-zero-tolerance

Joint Service Committee on Military Justice. (2008). Manual for Courts-Martial United States (2008 Edition). Department of Defense. Retrieved from http://www.loc.gov/rr/frd/Military_Law/pdf/MCM-2008.pdf

Kanuha, V. K. (2000). "Being” Native versus “Going Native”: Conducting Social Work Research as an Insider. "Being” Native versus "Going Native": Conducting Social Work Research as an Insider. Social Work, 45(5), 439-447.

Katz, L. S., Bloor, L. E., Cojucar, G., \& Draper, T. (2007). Women who served in Iraq seeking mental health services: Relationships between military sexual trauma, symptoms, and readjustment. Psychological Services, 4(4), 239249.http://doi.org/10.1037/1541-1559.4.4.239

Kelty, R., Kleykamp, M., \& Segal, D. R. (2010). The Military and the Transition to Adulthood. The Future of Children, 20(1), 181-207.

Kezar, A. (2002). Reconstructing Static Images of Leadership: An Application of Positionality Theory. The Journal of Leadership Studies, 8(3), 94-109. 
Krieger, N. (2003). Genders, sexes, and health: what are the connections--and why does it matter? International Journal of Epidemiology, 32(4), 652-657. http://doi.org/10.1093/ije/dyg156

Kvale, S. (1996). InterViews: An introduction to qualitative research interviewing. Thousand Oaks, CA: Sage.

Lincoln, Y.S., \& Guba, E.G. (2000). Paradigmatic Controversies, Contradictions, and emerging confluences. In N.K. Denzin \& Y.S. Lincoln (Eds.), Handbooks of qualitative research (2nd ed., pp. 163-188). Thousand Oaks, CA: Sage.

Lipsky, L. D., \& Burk, C. (2009). Trauma stewardship: An everyday guide to caring for self while caring for others. San Francisco, CA: Berrett-Koehler Publishers.

McMahon, S (2010). Rape Myth Beliefs and Bystander Attitudes Among Incoming College Students. Journal of American College Health, 59(1), 3-11.

Manis, J. G. (1974). Assessing the Seriousness of Social Problems. Social Problems, 22(1), 1-15. http://doi.org/10.2307/799563

Meier, M., \& Medjesky, C. (2018). The Office was asking for it: "that's what she said" as a joke cycle that perpetuates rape culture. Communication and Critical/Cultural Studies, 15(1), 2-17.

Mejia, P., Somji, A., Nixon, L., Dorfman, L., \& Quintero, F. (2015). What's missing from the news on sexual violence? Berkeley, CA. Retrieved from http://www.bmsg.org/sites/default/files/bmsg_issue22_sexual_violence_news.pdf Mertens, D. M. (2014). Research and Evaluation in Education and Psychology. In Research and evaluation in education and psychology: Integrating diversity with 
quantitative, qualitative, and mixed methods. (2nd Edition, pp. 1-46). Thousand Oaks, CA: Sage Publications Inc. Retrieved from http://www.sagepub.com/sites/default/files/upm-binaries/29985_Chapter1.pdf

Milat, A.J., King, L., Bauman, A. E., \& Redman, S. (2012). The concept of scalability: increasing the scale and potential adoption of health promotion interventions into policy and practice. Health Promotion International, 28(3), 285-298. https://doi.org/10.1093/heapro/dar097

Miles, D. (2011, November 28). Survey Shows Growing Gap Between Civilians, Military. American Forces Press Service. Washington D.C. Retrieved from http://content.govdelivery.com/accounts/USDOD/bulletins/1e626f

Military Justice International. (2015). Military Justice Guide to Sex Offender Registration Requirements. Retrieved November 24, 2015, from http://www.militaryjusticeinternational.com

Montgomery, N. (2013, February 27). Case dismissed against Aviano IG convicted of sexual assault. Stars and Stripes. Retrieved from http://www.stripes.com/news/air-force/case-dismissed-against-aviano-igconvicted-of-sexual-assault-1.209797

Morris, T. (2006). Social work research methods: four alternative paradigms. Thousand Oaks, Calif.: Thousand Oaks, Calif.: SAGE Publications.

Morton, T. A., Postmes, T., Haslam, S. A., \& Hornsey, M. J. (2009). Theorizing gender in the face of social change: Is there anything essential about essentialism? Journal of Personality and Social Psychology, 96(3), 653-664. 
http://dx.doi.org.proxy.lib.pdx.edu/10.1037/a0012966 Retrieved from http://stats.lib.pdx.edu/proxy.php?url=http://search.proquest.com.proxy.lib.pdx.ed u/docview/61449611 ? accountid=13265

Namrow, N. A., Hurley, Cook, P.J., M. M., Van Winkle, E. P., \& Hurley, M.M. (2016a). Overview Report: 2015 Service Academy Gender Relations Focus Groups. Fort Belvoir, VA. Retrieved from http://sapr.mil/public/docs/reports/MSA/APY_1415/SAGR_DoD2015_FocusGroupReport_ExSumm.pdf

Namrow, N. A., Hurley, M. M., Van Winkle, E. P., \& De Silva, S. (2016b). Overview Report: 2015 Military Investigation and Justice Experience Survey. Fort Belvoir, VA. Retrieved from http://sapr.mil/public/docs/reports/FY15_Annual/Annex_3_2015_MIJES_Report. pdf

National Sexual Violence Center (2010). What is Sexual Violence? (Fact Sheet).

Retrieved on April 4, 2018 from https://www.nsvrc.org/sites/default/files/201203/Publications_NSVRC_Factsheet_What-is-sexual-violence_1.pdf Navy Military Personnel Manual. MILPERSMAN 1070-320. Administrative Remarks (21 OCT 2019). Retrieved from https://www.public.navy.mil/bupersnpc/reference/milpersman/Documents/Whole\%20MILPERSMAN.pdf

Newman, E., Risch, E., \& Kassam-Adams, N. (2006). Ethical issues in trauma-related research: A review. Journal of Empirical Research on Human Research Ethics, $1(3), 29-46$.

NVivo qualitative data analysis software (2018); QSR International Pty Ltd. Version 12, 
O’Brien, C., Keith, J., \& Shoemaker, L. (2015). Don’t Tell: Military Culture and Male Rape. Psychological Services, 12(4), 357-365. https://doi.org/domain http://dx.doi.org/10.1037/ser0000049

Office of the Deputy Assistant Secretary of Defense. (2014) 2014 Demographics: Profile of the Military Community. Retrieved from

http://download.militaryonesource.mil/12038/MOS/Reports/2014-DemographicsReportpdf

Office of the Secretary of Defense. (1993). Memorandum to the Secretaries of the Army, Navy, and Air Force, and to the Chairman of the Joint Chiefs of Staff, from Les Aspin, Secretary of Defense, regarding the policy on homosexual conduct in the Armed Forces. Washington, DC: Secretary of Defense.

Office of the Secretary of Defense. (2013). Department of defense fact sheet: Secretary Hagel issues new initiatives to eliminate sexual assault, updates prevention strategy and releases 2013 Annual Report on sexual assault in the military. Retrieved from http://www.sapr.mil/public/docs/report/FY_DoD_DoD_SAPRO_Annual_Report Fact_Sheet.pdf

Office of Senator Kirsten Gillibrand. (2009). Comprehensive resource center for the Military Justice Improvement Act. Retrieved from http://www.gillibrand.senate.gov/mjia

Okros, A. (2009). Rethinking Diversity and Security. Commonwealth \& Comparative Politics. https://doi.org/10.1080/14662040903362990 
O’Neil, M., \& Morgan, P. (2010). American Perceptions of Sexual Violence. Frameworks Institute.

Oxford Reference (2011). Biological Essentialism. Retrieved 10 Mar. 2018, from http://www.oxfordreference.com/view/10.1093/oi/authority.20110803095507973

Payne, D. L., Lonsway, K. A., \& Fitzgerald, L. F. (1999). Rape myth acceptance: Exploration of its structure and its measurement using the Illinois Rape Myth Acceptance Scale. Journal of Research in Personality, 33, 27-68

Peralta, E. (May 6, 2013) Air Force Sexual Assault Prevention Chief Charged with Sexual Battery. NPR. Retrieved from http://www.npr.org/blogs/thetwoway/2013/05/06/181681231/air-force-sexual-assault-prevention-chief-chargedwith-sexual-battery

Planty, B. M., Langton, L., Krebs, C., Berzofsky, M., \& Smiley-McDonald, H. (2013). Female Victims of Sexual Violence, 1994-2010. Special Report. Washington, D.C.: Office of Justice Programs, U.S. Department of Justice. Retrieved from https://www.bjs.gov/content/pub/pdf/fvsv9410.pdf

Ponterotto, J. G. (2005). Qualitative Research in Counseling Psychology: A Primer on Research Paradigms and Philosophy of Science. Journal of Counseling.

Potter, S. J. (2016). Reducing sexual assault on campus: Lessons from the movement to prevent drunk driving. American Journal of Public Health. https://doi.org/10.2105/AJPH.2016.303082

Protect Our Defenders (n.d.). Frequently Asked Questions. Retrieved from http://www.protectourdefenders.com/frequently-asked-questions/\#offender 
Rand National Defense Research Institute. (2014). Sexual Assault and Sexual

Harassment in the Military. Washington D.C. Retrieved from http://sapr.mil/public/docs/reports/FY14_POTUS/FY14_DoD_Report_to_POTUS _Annex_1_RAND.pdf

Rand National Defense Research Institute (2014). Rand Military Workplace Study [RMWS]: Sexual Assault and Sexual Harassment in the U.S. Military. Retrieved from http://sapr.mil/public/docs/reports/FY14_Annual/FY14_Annual_Report_Annex_1 _RAND.pdf

Rape Abuse Incest National Network. [RAINN] (2009). Victims of Sexual Violence: Statistics. Retrieved from https://www.rainn.org/statistics/victims-sexual-violence Reinke, S. (2006). Abu Ghraib A Case of Moral and Administrative Failure. Public Integrity, 8(2), 135-147. http://doi.org/10.2753/PIN1099-9922080202

Rich, M. D., Utley, E. A., Janke, K., \& Moldoveanu, M. (2010). I'd Rather Be Doing Something Else:" Male Resistance to Rape Prevention Programs. The Journal of Men's Studies, 18(3), 268-288.

Rosenberg, M. (2016, June 30). Transgender People Will Be Allowed to Serve Openly in Military. The New York Times. Retrieved from http://www.nytimes.com/2016/07/01/us/transgender-military.html?_r=0

Rosenthal, L., \& Miller, K. (2013). The Data on Military Sexual Assault: What You Need to Know. Retrieved from 
http://americanprogress.org/issues/military/news/2013/07/23/70332/the-data-onmilitary-sexual-assault-what-you-need-to-know

Rostker, B. (2006). I Want You! The Evolution of the All-Volunteer Force. Santa Monica, CA: Rand Corporation. Retrieved from http://www.rand.org/pubs/monographs/MG265.html

Sayer, N. A., Spoont, M., Murdoch, M., Friedemann-Sanchez, G., Parker, L. E., Chiros, C., \& Rosenheck, R. (2009). a Qualitative Study of Determinants of PTSD treatment Initiation in Veterans. Psychiatry, 72(3).

Scheufele, D. A., \& Tewksbury, D. (2007). Framing, Agenda Setting, and Priming: The Evolution of Three Media Effects Models. Journal of Communication, 57, 9-20.

Seck, H. H. (2015). Navy Revamps Ethics Training in Wake of Submarine Shower Scandal. Retrieved from http://www.military.com/daily-news/2015/12/10/navyrevamps-ethics-training-in-wake-of-submarine-shower-scandal.html

Service Women's Action Network. (n.d.). Military Sex Offender Registration: Frequently Asked Questions. Retrieved November 25, 2015, from http://servicewomen.org/wpcontent/uploads/2012/08/MilitarySexOffenderRegistryFAQs.pdf

Sills, S., Pickens, C., Beach, K., Jones, L., Calder-Dawe, O., Benton-Greig, P., \& Gavey, N. (2016). Rape Culture and Social Media: Young Critics and a Feminist Counterpublic. Feminist Media Studies, 16(6), 935-951.

Social Work Degree Guide (2017). What is Macro Social Work? Retrieved from https://www.socialworkdegreeguide.com/faq/what-is-macro-social-work/ 
Solaro, E. (2006). Women in the Line of Fire: What You Should Know About Women in the Military. Emeryville, CA: Seal Press.

Spoehr, T \& Handy, T. (2018, February 13). The Looming National Security Crisis: Young Americans Unable to Serve in the Military. The Heritage Foundation. Retrieved on May 6, 2018 from https://www.heritage.org/defense/report/thelooming-national-security-crisis-young-americans-unable-serve-the-military

Steward, J. (2013). Understanding Male Survivors of Military Sexual Trauma. In V.A. Military Sexual Trauma Conference. Portland, OR.

Stinson, E. (2013). Military Sexual Trauma: Impact and Treatment. In V.A. Military Sexual Trauma Conference. Portland, OR.

Struckman-Johnson, C., \& Struckman-Johnson, D. (1992). Acceptance of male rape myths among college men and women. Sex Roles, 27, 85-100.

Suarez, E., \& Gadalla, T. M. (2010). Stop Blaming the Victim: A Meta-Analysis on Rape Myths. Journal of Interpersonal Violence, 25(11), 2010-2035. https://doi.org/10.1177/0886260509354503

Sue, D. W. (2010). Microaggressions in Everyday Life: Race, Gender and Sexual Orientation. Hoboken, N.J.: John Wiley \& Sons, Inc.

Sumagaysay, L. (2017, October 16). As \#MeToo trends, here's a list of sex scandals in tech and entertainment. The Mercury News. Retrieved from https://www.mercurynews.com/2017/10/16/as-metoo-trends-heres-a-list-of-sexscandals-in-tech-and-entertainment/ 
Surís, A., Lind, L., Kashner, T. M., Borman, P. D., \& Petty, F. (2004). Sexual assault in women veterans: an examination of PTSD risk, health care utilization, and cost of care. Psychosomatic Medicine, 66, 749-756. https://doi.org/10.1097/01.psy.0000138117.58559.7

Takacs, David. (2003). How Does Your Positionality Bias Your Epistemology? Thought \& Action, 19(1), 27-38.

Tsongas, N. (D, MA-5) \& Tardiff, R. (n.d.) Silent No more. [Blog post]. Retrieved from http://www.notinvisible.org/silent_no_more

Turchik, J. A., \& Wilson, S. M. (2010). Sexual assault in the U.S. military: A review of the literature and recommendations for the future. Aggression and Violent Behavior. https://doi.org/10.1016/j.avb.2010.01.005

United States Department of Defense (n.d.) Sexual Assault Prevention and Response Office. Retrieved from http://www.sapr.mil

United States Department of Veteran Affairs (November 30, 2017). Military Sexual Trauma. Retrieved from http://www.mentalhealth.va.gov/msthome.asp

Vanden Brook, Tom (2013 May 24) Sergeant busted for taking nude photos of cadets. USA Today. Retrieved from http://www.usatoday.com/story/news/nation/2013/05/22/sex-scandal-hits-westpoint/2352163/

Vanden Brook, T., \& Jackson, D. M. (2013, May 16). Obama says sexual assault crisis hurts national security. USA Today. Retrieved from http://www.usatoday.com/story/news/politics/2013/05/16/obama-hagel-military 
Venema, R. M. (2016). Police Officer Schema of Sexual Assault Reports: Real Rape, Ambiguous Cases, and False Reports. Journal of Interpersonal Violence, 31(5), 872-899. https://doi.org/10.1177/0886260514556765

Veterans Health Care Administration. (2013). Quick facts about VA's health care services for military sexual trauma. Retrieved from http://www.uscg.mil/worklife/docs/pdf/sapr_VA_Healthcare_Serices.pdf Walters, M.L., Chen J., \& Breiding, M.J. (2013). The National Intimate Partner and Sexual Violence Survey (NISVS): 2010 Findings on Victimization by Sexual Orientation.

Ward, V., House, A., \& Hamer, S. (2009). Developing a Framework for Transferring Knowledge into Action: A Thematic Analysis of the Literature. Journal of Health Services Research \& Policy, 14(3), 156-164.

Williams, I., \& Bernstein, K. (2011). Military Sexual Trauma Among U.S. Female Veterans. Archives of Psychiatric Nursing. http://doi.org/10.1016/j.apnu.2010.07.00

Wire Reporters (2015, December 3). Defense Secretary tells military to open all combat jobs to women. Chicago Tribune. Retrieved from http://www.chicagotribune.com/news/nationworld/ct-military-combat-jobswomen-20151203-story.html

Wooten, N.R. (2015). Military Social Work: Opportunities and Challenges for Social Work Education. Journal of Social Work Education. 51(Suppl 1), S6-S25. 
Yamawaki, N. (2007). Rape Perception and the Function of Ambivalent Sexism and Gender-Role Traditionality. Journal of Interpersonal Violence, 22(4), 406-423. https://doi.org/https://doi-org.proxy.lib.pdx.edu/10.1177/0886260506297210

Yoder, J. D. (1991). Rethinking Tokenism: Looking beyond numbers. Gender and Society, 5(2), 178-192.

Yoder, J. D., Adams, J., \& Prince, H. T. (1983). The Price of a Token. Journal of Political and Military Sociology, 11(2), 325-337.

113th Congress. (2013). Sexual Assault in the Military. Washington D.C. Retrieved from https://www.armedservices.senate.gov/imo/media/doc/sexualassaultsinmilitary_su bcomm_hearing_031313.pdf

113th Congress. (2014). The relationship between military sexual assault, post-traumatic stress disorder and suicide, and on the Department of Defense and on the Department of Veteran Affairs Medical Treatment and management of victims of sexual trauma. Washington D.C. Retrieved from https://www.armedservices.senate.gov/imo/media/doc/91318.pdf

Zacharek, S., Docterman, E. \& Sweetland Edwards, H. (2017, December 18). Person of the Year. The Silence Breakers: The Voices that Launched a Movement. Time Magazine. Retrieved from http://time.com/time-person-of-the-year-2017-silencebreakers/ 


\section{Appendix A: Participant Screening Tool}

*This word version was converted to a Qualtrics survey for the participants (some yes/no questions will have skip logic for a more user-friendly and streamlined survey experience)

\section{INTRODUCTION}

Thank you for expressing an interest in participating in this study about military sexual trauma (MST), which is a VA term to describe incidents of sexual harassment and sexual assault incurred during military service.

I am interested in exploring not only your experiences of sexual harassment and sexual assault incurred during active duty, but your perception of the military's prevention and response efforts, as well as your recommendations to improve them.

There are risks of stress, and emotional distress, before, during or after your interview. Certain questions may trigger unpleasant memories or experiences, to include, but not limited to, symptoms of post-traumatic stress disorder (PTSD), anxiety, or depression. I encourage you to utilize the resources at the end of this document following local (Portland metro area), and national resources as an additional form of support. For more information about risks and discomforts, don't hesitate to contact the investigator.

In addition to determining your eligibility for the study, I will also be asking demographic questions to ensure that we have a strong diversity of participants to include but not limited to:

- military service branch

- gender identity

- sexual orientation

- race

- ethnicity

This brief survey will take no more than 10-15 minutes to complete. For your reference, please find below the definitions the military currently uses for sexual harassment and sexual assault.

The military defines sexual harassment as "a form of sex discrimination that involves unwelcome sexual advances, requests for sexual favors, and other verbal or physical conduct of a sexual nature when:

- submission to such conduct is made either explicitly or implicitly a term or condition of a person's job, pay, or career

- submission to or rejection of such conduct by a person is used as a basis for career or employment decisions affecting that person 
- such conduct has the purpose or effect of unreasonably interfering with an individual's work performance or creates an intimidating, hostile, or offensive working environment". ${ }^{47}$

The military defines sexual assault as "Any person subject to this chapter who causes another person of any age to engage in a sexual act by:

- using force against that other person

- causing grievous bodily harm to any person

- threatening or placing that other person in fear that any person will be subjected to death, grievous bodily harm, or kidnapping

- rendering another person unconscious

- administering to another person by force or threat of force, or without the knowledge or permission of that person, a drug, intoxicant, or similar substance and thereby substantially impairs the ability of that other person to appraise or control conduct". ${ }^{48}$

\section{MILITARY SERVICE EXPERIENCE}

\section{Q1: What years did you serve on active duty? (ex 2001-2004)}

\section{Q2: What military branch did you serve with while on active duty? (please check one)}

Army

Navy

Air Force

Marine Corps

Coast Guard

\footnotetext{
${ }^{47}$ Department of Defense. (2014). Provisional Statistical Data on Sexual Assault Fiscal Year 2014: Report to the President of the United States on SAPR. Retrieved from http://sapr.mil/public/docs/reports/FY14_POTUS/FY14_DoD_Report_to_POTUS_Appendix_A.pdf

${ }^{48}$ Joint Service Committee on Military Justice. (2008). Manual for Courts- Martial United States (2008 Edition). Department of Defense. Retrieved from http://www.loc.gov/rr/frd/Military_Law/pdf/MCM2008.pdf
} 
III. EXPERIENCE WITH SEXUAL HARASSMENT AND SEXUAL ASSAULT IN THE MILITARY

Q3: When you served on active duty, did you directly experience at least one incident of sexual harassment?

Yes

No

Q4: Did the sexual harassment incident(s) occur while: (please check all that apply)

Military Service Academy (ex. West Point, Naval Academy, etc.)

CONUS (Within the Continental U.S.: "Stateside "Duty station)

OCONUS (Outside Continental U.S.: Overseas Duty Station)

Deployment/Tour of Duty

Other (please list)

Q5: Were any of the sexual harassment perpetrators a fellow service member?

Yes

No

Q6: Were any of the perpetrators...? (please choose all that apply)

in your unit?

a direct supervisor?

in your chain of command

other (please list)

Q7: What was the rank of the perpetrator(s) at the time of the sexual harassment incident(s)? (please check all that apply) 
E1-E4

E5-E6

E7-E9

WO1-CWO2

$\mathrm{CW}[0] 3-\mathrm{CW}\{0\} 5$

O1-O3

O4-O6

O7-O10

Q8: What was your rank at the time of the sexual harassment incident(s) (please check all that apply)

E1-E4

E5-E6

E7-E9

WO1-CWO2

$\mathrm{CW}[0] 3-\mathrm{CW}\{0\} 5$

O1-O3

O4-O6

O7-O10

Q9: When you served on active duty, did you directly experience at least one incident of sexual assault?

Yes

No

Q10: Did the incident(s) occur while stationed: (please check all that apply)

Military Service Academy (ex. West Point, Naval Academy, etc.)

CONUS (Within the Continental U.S.:"Stateside”Duty station)

OCONUS (Outside Continental U.S.: Overseas Duty Station) 
Deployment/Tour of Duty

Other (please list)

Q11: Were any of the sexual assault perpetrators a fellow service member?

Yes

No

Q12: Were any of the perpetrators...? (please choose all that apply)

in your unit?

a direct supervisor?

in your chain of command

other (please list)

Q13: What was the rank of the perpetrator(s) at the time of the sexual assault incident(s)? (please check all that apply)

E1-E4

E5-E6

E7-E9

WO1-CWO2

$\mathrm{CW}[0] 3-\mathrm{CW}\{0\} 5$

$\mathrm{O} 1-\mathrm{O} 3$

O4-O6

$\mathrm{O} 7-\mathrm{O} 10$

Q14: What was your rank at the time of the sexual assault incident(s) (please check all that apply)

E1-E4

E5-E6

E7-E9

WO1-CWO2

$\mathrm{CW}[0] 3-\mathrm{CW}\{0\} 5$

O1-O3 
O4-O6

O7-010

Q15: Were any of the perpetrators a fellow service member?

Yes

No

Q16: Were any of the perpetrators...? (please choose all that apply)

in your unit?

a direct supervisor?

in your chain of command

other (please list)

Q17: What was the rank of the perpetrator(s) at the time of the sexual assault incident(s)? (please check all that apply)

E1-E4

E5-E6

E7-E9

WO1-CWO2

$\mathrm{CW}[0] 3-\mathrm{CW}\{0\} 5$

O1-O3

O4-06

O7-O10

Q18: What was your rank at the time of the sexual assault incident(s) (please check all that apply)

E1-E4

E5-E6

E7-E9

WO1-CWO2

CW[0]3-CW $\{0\} 5$

O1-O3

O4-O6 
O7-O10

\section{PERSONAL DEMOGRAPHICS}

Q19: How old are you?

Q20: Do you identify as transgender?

Yes

No

Q21: In terms of gender identity, do you identify as?

(please check any/all that apply)

Woman

Man

Non-binary

Non-gender conforming

Not listed above, I identify as (please list)

I prefer not to answer

Q22: In terms of sexual orientation, do you identify as? (please check your preference)

Heterosexual/Straight

Lesbian

Gay

Queer

Bisexual

Asexual

Pansexual 
Not listed above, I identify as

(please list)

I prefer not to answer

\section{Q23: In terms of race, do you identify as? (please check all that apply)}

White

Black or African American

Native American or Alaska Native

Asian or Asian American

Native Hawaiian or Other Pacific Islander

Middle Eastern/North African

Multiracial

Not listed above, I identify as (please list)

I prefer not to answer

Q24: In terms of ethnicity, do you identify as? (please check all that apply)

Hispanic/Latino(a)

Non-Hispanic/Latino(a)

Not listed above, I identify as (please list)

Multiethnic

I prefer not to answer

\section{Q25: Education level (please check the highest level attained)}

Less than high school

High School Diploma 
GED

Associates Degree

Bachelor's Degree

Master's Degree

Doctoral Degree

(ex. Ph.D, Ed.D., M.D., J.D)

Q26: Location (the state you currently reside in)

\section{MILITARY SEXUAL TRAUMA (MST) SURVIVOR RESOURCES}

Portland State University (PSU) student resources:

Women's Resource Center (WRC): 503-725- 5672

http://www.pdx.edu/wrc/contact

Queer Resource Center (QRC): 503-725-9742

https://www.pdx.edu/queer/interpersonal-violence-ipv-resources

Veterans Resource Center (VRC): 503-725-9807

https://www.pdx.edu/veterans/vrc

Student Health and Counseling Center (SHAC): 503-725- 2800

http://www.pdx.edu/shac/counseling

\section{Portland (OR) Metro Resources:}

Portland Vet Center: 503-688- 5361

Multnomah County Crisis Line: 503-988- 4888.

V.A. Women's Health Center: 503-402- 2852

http://www.portland.va.gov/wvhc.asp

\section{National (U.S.A.) Resources:}

Veteran Crisis Line (1-800-273-8255 and Press 1) for help.

Military OneSource: 1-800- 342-9647

http://www.militaryonesource.mil

Returning Veterans' Project: 503-954- 2259

http://www.returningveterans.org/providers/find 
Veteran Centers

1-877-927- 8387

https:/www.va.gov/directory/guide/vetcenter.asp?isFlash=0 


\title{
Appendix B: Informed Consent, Phone Interview
}

\author{
The Portland State University \\ Consent to Participate in Research (Phone Interview Version) \\ Examining the Narratives of Military Sexual Trauma (MST)
}

December 11, 2018

\section{Introduction}

You are being asked to participate in the research study "Examining the Narratives of Military Sexual Trauma (MST)". It is being conducted by Maria Carolina GonzàlezPrats, who is a doctoral student in the Social Work and Social Research program at Portland State University (PSU) in Portland, Oregon. Carolina is the study's Principal Investigator and is under the supervision of Dr. Christina Nicolaidis from PSU's School of Social Work.

This research is studying the military's sexual assault prevention and response efforts. The in-depth interview will consist of questions asking you about your direct experience with sexual harassment and/or sexual assault during your military service, and your military unit's response to the incident(s). I will draw from interviews from service members from various armed forces branches, including the Army, Air Force, Navy, Marine Corps and Coast Guard.

This form will explain the research study, as well as explain the possible risks and the possible benefits to you. I encourage you to talk with your family and/or friends before you decide to take part in this research study. If you have any questions, please ask the study's investigator.

\section{What will happen if you decide to participate?}

If you agree to participate, the following things will happen:

- You will take part in an interview.

- Your interview will be recorded digitally.

\section{How long will the study last?}

Participation in this study will take a total of $1-1 \frac{1}{2}$ hours for the interview.

\section{What are the risks or side effects of being in this study?}

There are risks of stress, emotional distress, inconvenience and the possible loss of privacy and confidentiality associated with participating in a research study. For example, someone in your home (friend[s] or family member[s]) may walk in during the course of the interview. 
Certain questions may trigger unpleasant memories or experiences, to include, but not limited to, symptoms of post-traumatic stress disorder (PTSD), anxiety, or depression. I want to reassure you that you have the choice not to answer (a) certain question(s) or stop the interview at any point if you feel uncomfortable. Additionally, I encourage you to utilize the following local (Portland metro area), and national resources as an additional form of support. For more information about risks and discomforts, don't hesitate to contact the investigator.

\section{MILITARY SEXUAL TRAUMA (MST) RESOURCES FOR SURVIVORS}

\section{Mental Health and Counseling: National/U.S.A. Resources}

- Rape, Abuse \& Incest National Network: RAINN (1-800-656-HOPE [4673]) https://www.rainn.org/about-rainn

- 1 in 6 https://1in6.org/

- Veteran Crisis Line (1-800-273-8255 and Press 1) for help.

- Military OneSource: 1-800- 342-9647 http://www.militaryonesource.mil

- Returning Veterans' Project: 503-954- 2259 http://www.returningveterans.org/providers/find

- Veteran Centers: 1-877-927-8387 https://www.va.gov/directory/guide/vetcenter.asp?isFlash $=0$

- Trans Lifeline: 1-877-565-8860 https://www.translifeline.org/ 
Mental Health and Counseling: Portland, OR Metro Resources

- Portland Vet Center: 503-688- 5361

- Multnomah County Crisis Line: 503-988- 4888

- V.A. Women's Health Center: 503-402- 2852 http://www.portland.va.gov/wvhc.asp

Mental Health and Counseling: $\underline{P S U \text { student resources }}$

- Women's Resource Center (WRC): 503-725- 5672 http://www.pdx.edu/wrc/contact

- Student Health and Counseling Center (SHAC): 503-725- 2800 http://www.pdx.edu/shac/counseling

National Resource Directory for Military Veterans and their families https://nrd.gov/

Veteran and Military Service Organizations https://www.va.gov/vso/VSO-Directory.pdf

Housing Assistance:

- National Call Center for Homeless Veterans (1-877-4AID-VET)

- HUD-VASH program for homeless veterans

https://www.va.gov/homeless/hud-vash eligibility.asp

V.A. Benefits and Health Care

https://www.va.gov/health-care/how-to-apply/

Transition Care Management (OEF/OIF/OND)

https://www.oefoif.va.gov/map.asp

\section{V.A. Disability Benefits}

https://www.va.gov/disability/eligibility/ 


\section{Veterans Pension Benefits}

https://www.va.gov/pension/eligibility/

\section{Education and Training}

- Compensated Work Therapy (CWT)

https://www.va.gov/HEALTH/cwt/veterans.asp

- Applying for VA Educational Benefits:1-888-442-4551

https://www.va.gov/education/how-to-apply/

- Vocational Rehabilitation and Employment

https://www.benefits.va.gov/vocrehab/index.asp

- Post-9/11 GI Bill (Chapter 33)

https://www.va.gov/education/about-gi-bill-benefits/post-9-11/

- Montgomery GI Bill Active Duty: 1-888-442-4551)

https://www.va.gov/education/about-gi-bill-benefits/montgomery-active-duty/

- Montgomery GI Bill Selected Reserve: 1-888-442-4551),

https://www.va.gov/education/about-gi-bill-benefits/montgomery-selected-reserve/

\section{What are the benefits to being in this study?}

The information gained from your participation in this research study may contribute to the knowledge base about the survivors of military sexual trauma, which historically has been

minimized or left out altogether in the military's prevention and response efforts. Such knowledge may inform future efforts to decrease incidents of sexual harassment and sexual assault in the military.

\section{How will my information be kept confidential?}

I will take the proper measures to protect the security of your personal information. With your permission I will be recording the interview but I will be identifying you on the recorder and my notes as a four-digit alpha numeric code (ex. d7bf). The only identifying documents I will have with your name on it will be this consent form and a participant key (matching the letter with your name), which will be secured separately in restricted drive maintained by PSU's Regional Research Institute (RRI). 
Information contained in your study records will only be shared with the supervising professor and a researcher assistant. The Portland State University Institutional Review Board (IRB) that oversees human subject research and/or other entities may be permitted to access your records, and there may be times when we are required by law to share your information. The consent form and all identifying information will be destroyed upon the completion of the research study.

As the investigator, it is my legal obligation to report child abuse, child neglect, elder abuse, harm to self or others or any life-threatening situation. If you tell me, then I will need to report the information to the appropriate authorities.

The in-person interview and/or telephone interview (if needed) will be audio-taped for an accurate record of the conversation. The conversation will then be transcribed. Your name will not be used in any published reports about this study.

\section{Will I be paid for taking part in this study?}

No.

\section{Can I stop being in the study once I begin?}

Your participation in this study is completely voluntary. You have the right to choose not to participate or to withdraw your participation at any point in this study.

Whom can I call with questions or complaints about this study?

If you have any questions, concerns or complaints at any time about the research study, Maria Carolina Gonzalez-Prats, will be glad to answer them at 971-404- 9654 or mgonza2@pdx.edu

Additionally, if you have a complaint or concern about the above primary investigator, you may reach the supervising professor, Christina Nicolaidis at (503) 725-9603 or nicol22@pdx.edu

\section{Whom can I call with questions about my rights as a research participant?}

If you have questions regarding your rights as a research participant, you may call the PSU Office for Research Integrity at (503) 725-2227 or 1(877) 480-4400. The ORI is the office that supports the PSU Institutional Review Board (IRB). The IRB is a group of people from PSU and the community who provide independent oversight of safety and ethical issues related to research involving human participants. For more information, you may also access the IRB website at https://sites.google.com/a/pdx.edu/research/integrity. 


\section{Whom can I call with questions about my rights as a research participant?}

If you have questions regarding your rights as a research participant, you may call the PSU Office for Research Integrity at (503) 725-2227 or 1(877) 480-4400. The ORI is the office that supports the PSU Institutional Review Board (IRB). The IRB is a group of people from PSU and the community who provide independent oversight of safety and ethical issues related to research involving human participants. For more information, you may also access the IRB website at https://sites.google.com/a/pdx.edu/research/integrity.

\section{CONSENT}

You are making a decision whether to participate in this study. Your signature below indicates that you have read the information provided (or the information was read to you). By signing this consent form, you are not waiving any of your legal rights as a research participant.

You have had an opportunity to ask questions and all questions have been answered to your satisfaction. By signing this consent form, you agree to participate in this study, and to have your interview audio recorded. A copy of this consent form will be provided to you.

Are you willing to participate in this research as it has been explained to you? If so, please sign your name and fill in today's date.

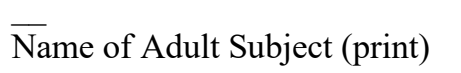

$\overline{\text { Signature of Adult Subject }}$

$\overline{\text { Date }}$

\section{INVESTIGATOR SIGNATURE}

This research study has been explained to the participant and all of his/her questions have been answered. The participant understands the information described in this consent form and freely consents to participate.

Maria Carolina González-Prats

Name of Investigator/ Research Team Member (type or print)

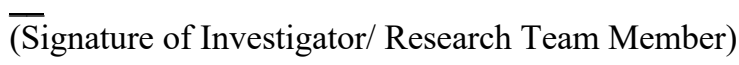

$\overline{\text { Date }}$ 


\section{Appendix C: Interview Guide}

\section{Introduction:}

Hello, my name is Carolina and I'm the principal investigator for this study. You have a unique and valuable perspective that can be used to improve the military's prevention and response efforts to incidents of sexual harassment and sexual assault.

I'm going to ask you some questions to learn about your military service, specifically the incident(s) of sexual harassment and/or sexual assault you experienced, and how you perceived your unit and chain of command's response towards the harassment and sexual assault.

You can take as much time as you need to answer. You can choose to skip a question if you want to come back to it later or if you do not wish to answer.

(For those interviews taking place over the phone) Additionally, in the event, a friend, family member or roommate comes into your home and you feel you are not able to speak freely, please let me know, and we can reschedule, if that would make you feel more comfortable.

Before we start the interview, do you have any questions for me? Are you ready to start?

Just so you know, this will be the structure of the interview. I will begin with some preliminary questions about your military service, before moving onto your perceptions of the harassment and assault training you received in the military. I will then transition to sexual harassment and/or sexual assault during your service, and then conclude with the recommendations to improve the prevention and response efforts of the military.

\section{Icebreaker Questions}

- How old were you when joined the military and what motivated you to do so?

- What was your military occupational specialty (MOS) in the service?

\section{Questions in re: Perceptions of Military's Policies in re MSH/MSA}

II-a. When was the first time you heard about the military's policy towards sexual harassment and sexual assault? [Was it when you were being recruited, basic training/boot camp or your first duty station?] 
II-b. How was this communicated? (i.e. formal training, briefings from your chain of command? Your military peers?)

II-c. If you went through formal sexual harassment or sexual assault prevention training, can you tell me more about it?

Depending on the participant's narrative... use the below probes for follow up/clarification

- Was the training mandatory?

- Did you feel that this training was effective? Why/why not?

- What messages did you walk away with?

I'm going to switch to your direct experience with sexual harassment and/or sexual assault...

\section{Incident(s) of MSH and/or MSA/and Potential Role their identity played}

III-a. In your questionnaire you indicated that you were sexually harassed and/or sexual assaulted while on active duty; I'm so sorry this happened to you, would you be willing to share with me what happened?

III-b. You also mentioned in the questionnaire that you identify as (gender identity/sexual orientation, race/ethnicity, etc.), do you feel that it played a factor in your harassment and/or assault? How so?

III-c. Based on your own direct experiences with military sexual trauma (MST), were the military's formal policies about sexual harassment and/or rape, and sexual assault consistent with the reality of how you were treated by your chain of command and military unit? Why or why not?

Depending on the participant's narrative... use the below probes for follow up/clarification

- Was the perpetrator a peer, supervisor, someone in your chain of command? (If so, do you remember their rank or job position?)

- Did it occur more than once?

- Did you tell anyone about the incident? (a friend, supervisor, etc.)

- If so, what happened when you did so? (any social/professional backlash)

- How did the incident(s) affect you personally, professionally?

- How could have you been supported better? 


\section{Recommendations) to improve military's prevention/response efforts}

Another goal of this research study is to contribute to the improvement of the SAPR training in the military.

IV-a. If you were given the task of updating and improving the Sexual Assault Prevention and Response (SAPR) training in the military, what might that look like?

Depending on the participant's narrative... use the below probes for follow up/clarification

- Who would your protagonists or characters be and what would they be doing?

- What are 1-2 messages would you want the service members to walk away in that training?

- What recommendations would you give to facilitators?

\section{Reflections and wrap-up}

$\mathrm{V}$-a. We have talked a lot about your experiences in the military and with MSH/MSA in particular. Thinking back on all you told me today, what is the most important message you want me to know about your story?

V-b. Is there anything else that you would like to share that I didn't ask about?

Thanks again for your time, participating in this study and sharing your insight about your experiences in the military. It will be extremely helpful to develop and recommend a more survivor-centered approach to the military's sexual assault prevention and response programs. 